\title{
Diarylamine synthesis via desulfinylative Smiles rearrangement
}

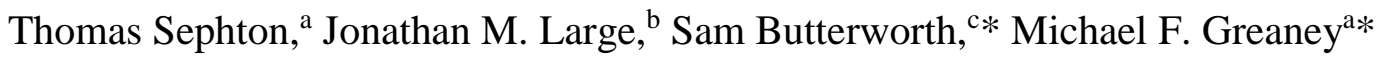

a Dept. of Chemistry, University of Manchester, Oxford Rd, Manchester, M13 9PL, UK.

${ }^{\mathrm{b}}$ LifeArc, Accelerator Building, Open Innovation Campus, Stevenage, SGI 2FX, UK.

${ }^{\mathrm{c}}$ Division of Pharmacy and Optometry, School of Health Sciences, Manchester Academic Health Sciences

Centre, University of Manchester, Manchester, M13 9PL, UK.

*michael.greaney@manchester.ac.uk

*sam.butterworth@manchester.ac.uk

\section{Supporting Information}

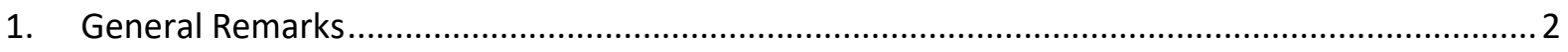

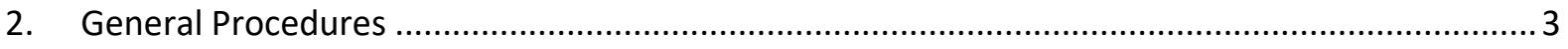

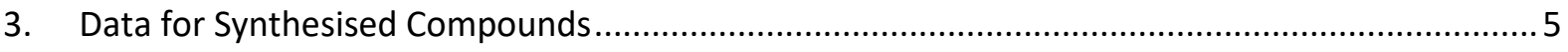

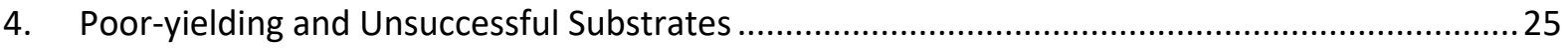

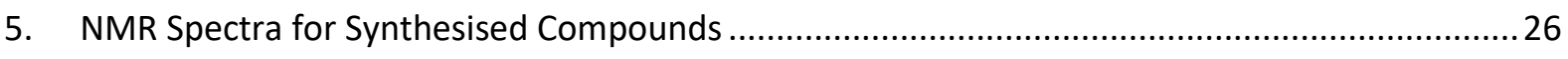

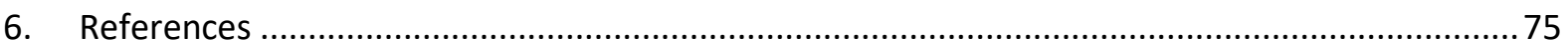




\section{General Remarks}

All solvents and reagents were purchased from Sigma Aldrich, Thermo Fisher Scientific, Apollo Scientific or Fluorochem and were used as received without further purification. Flash column chromatography was performed using either Biotage Snap Ultra cartridges or Biotage Sfar Silica cartridges on a Biotage Isolera automated column. ${ }^{1} \mathrm{H},{ }^{13} \mathrm{C}$ and ${ }^{19} \mathrm{~F}$ NMR spectroscopy were recorded on either $400 \mathrm{MHz}$ or $500 \mathrm{MHz}$ Bruker Avance NMR spectrometers. Chemical shifts ( $\delta$ ) are reported in parts per million (ppm) and multiplicities are reported as either singlets $(\mathrm{s})$, doublets $(\mathrm{d})$, triplets $(\mathrm{t})$, quartets $(q)$ or multiplets $(m)$. Coupling constants $(J)$ are reported in Hertz $(\mathrm{Hz})$. All ${ }^{1} \mathrm{H}$ NMR and ${ }^{13} \mathrm{C}$ NMR shifts were referenced to the residual solvent peak of $\mathrm{CDCl}_{3}\left({ }^{1} \mathrm{H}\right.$ referenced to $7.26 \mathrm{ppm}$ and ${ }^{13} \mathrm{C}$ referenced to $77.16 \mathrm{ppm}), \mathrm{DMSO}-\mathrm{d}_{6}\left({ }^{1} \mathrm{H}\right.$ referenced to $2.50 \mathrm{ppm}$ and ${ }^{13} \mathrm{C}$ referenced to $39.52 \mathrm{ppm}$ ) and acetone- $d_{6}\left({ }^{1} \mathrm{H}\right.$ referenced to $2.06 \mathrm{ppm}$ and ${ }^{13} \mathrm{C}$ referenced to $\left.29.84 \mathrm{ppm}\right)$. All ${ }^{19} \mathrm{~F}$ chemical shifts were unadjusted from raw data. 2D heteronuclear single quantum coherence (HSQC), heteronuclear multiple bond correlation (HMBC) and homonuclear correlation spectroscopy (COSY) NMR spectroscopy was used to assist the assignment of signals. High resolution mass spectrometry (HRMS) was recorded on a Waters QTOF, using either ESI or APCI as ionisation methods. Thin layer chromatography (TLC) was carried out using commercially available coated TLC plates and spots were illuminated either by UV light $(254 \mathrm{~nm})$ or by staining the plate with a $\mathrm{KMnO}_{4}$ solution. Compound names are those generated by ChemBioDraw ${ }^{\mathrm{TM}}$ (CambridgeSoft) following International Union of Pure and Applied Chemistry (IUPAC) nomenclature. Novel compounds are labelled in italics. Melting points (MPs) were recorded on a Griffin melting point apparatus to the nearest degree. Reactions which proceeded under microwave irradiation were performed in a Biotage Initiator Microwave Synthesizer. 


\section{General Procedures}

\section{General Procedure A for the Synthesis of N-Aryl Sulfinamides}<smiles>COS(=O)c1ccc([N+](=O)[O-])cc1</smiles>

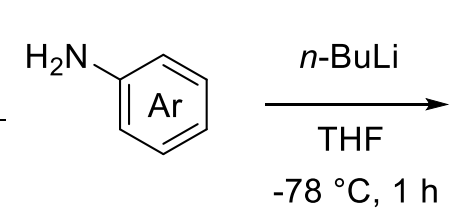<smiles>O=[N+]([O-])c1ccc(S(=O)Nc2ccccc2)cc1</smiles>

Carried out by adaptation of a literature procedure. ${ }^{1} \mathrm{~A} 2-5 \mathrm{~mL}$ microwave vial was charged with the corresponding aniline $(2.5 \mathrm{mmol})$ and the vial was evacuated under vacuum and filled with nitrogen. Anhydrous THF $\left(2 \mathrm{~mL}\right.$ ) was added and the solution was stirred at $-78^{\circ} \mathrm{C}$. $n$-BuLi $(1.6 \mathrm{M}$ in hexanes, 1 $\mathrm{mL}, 1.6 \mathrm{mmol}$ ) was added dropwise and the mixture was stirred at $-78^{\circ} \mathrm{C}$ for 20 minutes. A second 2-5 $\mathrm{mL}$ microwave vial was charged with methyl sulfinate 8 (200 mg, 2 mmol), evacuated under vacuum and filled with nitrogen. Dry THF $(1.5 \mathrm{~mL})$ was added, and the resulting solution was added dropwise to the reaction mixture, which was then stirred for $1-2$ hours at $-78^{\circ} \mathrm{C}$. The reaction mixture was then quenched with saturated aqueous $\mathrm{NaHCO}_{3}(3 \mathrm{~mL})$ and extracted with EtOAc $(3 \times 20 \mathrm{~mL})$. The organic layer was then washed with saturated aqueous $\mathrm{NaHCO}_{3}(3 \times 20 \mathrm{~mL})$ and dried with anhydrous $\mathrm{MgSO}_{4}$. This was then filtered, and concentrated in vacuo to afford the crude product, which was purified by flash column chromatography.

\section{General Procedure B for the Desulfinylative Smiles Rearrangement}

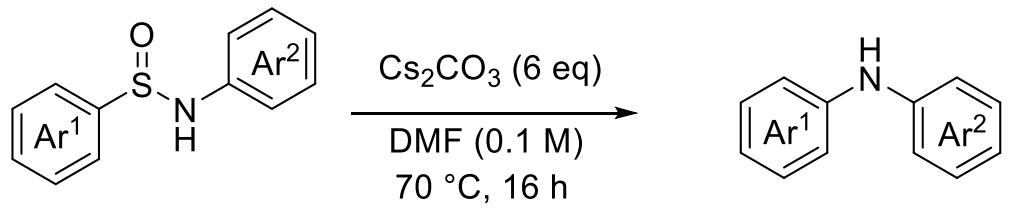

A 2-5 $\mathrm{mL}$ microwave vial was charged with the corresponding sulfinamide $(0.2 \mathrm{mmol})$ and $\mathrm{Cs}_{2} \mathrm{CO}_{3}(392$ $\mathrm{mg}, 1.2 \mathrm{mmol}$ ), evacuated under vacuum and filled with nitrogen. Anhydrous DMF ( $2 \mathrm{~mL}$ ) was added, and the mixture was stirred in a pre-warmed oil bath at $70^{\circ} \mathrm{C}$ for $16 \mathrm{~h}$. The vial was opened, the reaction mixture was dissolved in EtOAc $(20 \mathrm{~mL})$, washed with $1 \mathrm{M}$ aqueous $\mathrm{HCl}(2 \times 20 \mathrm{~mL})$ and $10 \%(\mathrm{w} / \mathrm{v})$ aqueous $\mathrm{LiCl}(2 \times 20 \mathrm{~mL})$. The aqueous layer was then extracted with EtOAc $(3 \times 20 \mathrm{~mL})$, and the combined organic layers were dried with anhydrous $\mathrm{MgSO}_{4}$, filtered and concentrated in vacuo. The crude product was then purified by flash column chromatography, affording the pure product.

\section{General Procedure C for the Intermolecular $S_{N} A r$ Control Reaction}

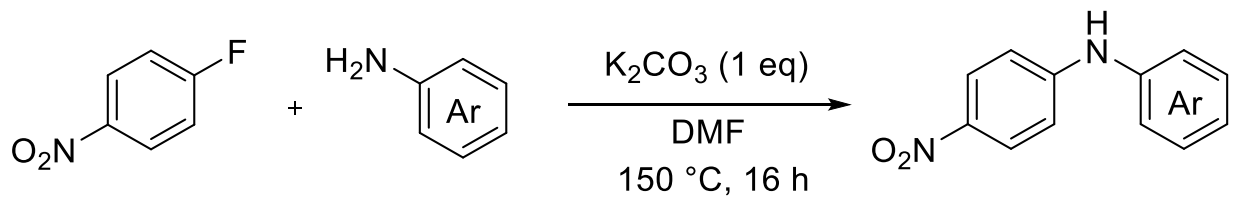

Carried out according to a literature procedure. ${ }^{2}$ A 2-5 mL microwave vial was charged with 1-fluoro4-nitrobenzene (141 mg, $1 \mathrm{mmol}$ ) and $\mathrm{K}_{2} \mathrm{CO}_{3}(140 \mathrm{mg}, 1 \mathrm{mmol})$, evacuated under vacuum and filled with nitrogen. Anhydrous DMF $(2 \mathrm{~mL})$ and the corresponding aniline $(1.1 \mathrm{mmol})$ were added and the reaction mixture was heated to reflux for $16 \mathrm{~h}$. After $16 \mathrm{~h}$, the reaction was monitored by TLC (30:70 
EtOAc:hexane). No product was observed, and this was confirmed by NMR analysis of the crude product. No purification was necessary.

\section{General Procedure D for the One-Pot Desulfinylative Cross-Coupling}<smiles>COS(=O)c1ccc([N+](=O)[O-])cc1</smiles>
a) $n$-BuLi (3.2 eq) $\mathrm{THF},-78^{\circ} \mathrm{C}, 1 \mathrm{~h}$ b) quench $(\mathrm{MeOH})$
c) DMF, $70^{\circ} \mathrm{C}, 16 \mathrm{~h}$ $30 \%$<smiles>Cc1cccc(Nc2ccc([N+](=O)[O-])cc2)c1</smiles>

A $50 \mathrm{~mL}$ round-bottomed flask was charged with the corresponding aniline $(2.5 \mathrm{mmol})$, evacuated under vacuum and filled with nitrogen. Anhydrous THF $(2 \mathrm{~mL})$ was added and the solution was stirred at $-78^{\circ} \mathrm{C}$. n-BuLi (1.6 $\mathrm{M}$ in hexanes, $2 \mathrm{~mL}, 3.2 \mathrm{mmol}$ ) was added dropwise and the mixture was stirred at $-78{ }^{\circ} \mathrm{C}$ for 20 minutes. A second $50 \mathrm{~mL}$ round-bottomed flask was charged with methyl sulfinate 8 (200 mg, $2 \mathrm{mmol})$, evacuated under vacuum and filled with nitrogen. Anhydrous THF (1.5 mL) was added, and the resulting solution was added dropwise to the reaction mixture, which was then stirred for $1 \mathrm{~h}$ at $-78^{\circ} \mathrm{C}$. The reaction mixture was quenched with $\mathrm{MeOH}(3 \mathrm{~mL})$ and the crude product was concentrated in vacuo. The flask was then filled with nitrogen, and the crude product dissolved in anhydrous DMF $(5 \mathrm{~mL})$. The flask was then stirred at $70^{\circ} \mathrm{C}$ in a pre-warmed oil bath for $16 \mathrm{~h}$. After this, the mixture was extracted with EtOAc $(3 \times 20 \mathrm{~mL})$, washed with $1 \mathrm{M}$ aqueous $\mathrm{HCl}(2 \times 20 \mathrm{~mL})$ and $10 \%$ $(\mathrm{w} / \mathrm{v})$ aqueous $\mathrm{LiCl}(3 \times 20 \mathrm{~mL})$. The organic layers were combined and dried with anhydrous $\mathrm{MgSO}_{4}$. This was filtered, and then concentrated in vacuo to afford the crude product, which was purified by flash column chromatography to afford the pure product.

\section{General Procedure E for the Microwave Reaction}

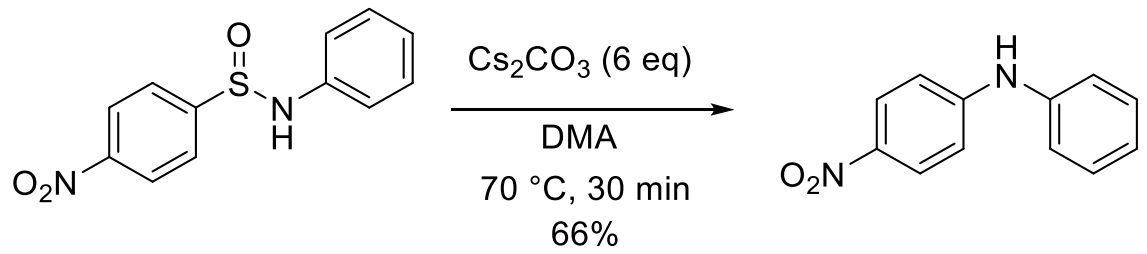

A sealed 2-5 $\mathrm{mL}$ microwave vial was charged with the corresponding sulfinamide $(0.2 \mathrm{mmol})$ and $\mathrm{Cs}_{2} \mathrm{CO}_{3}(392 \mathrm{mg}, 1.2 \mathrm{mmol})$, evacuated under vacuum and filled with nitrogen. Anhydrous DMA ( $\left.2 \mathrm{~mL}\right)$ was added, and the mixture was placed in a Biotage Initiator Microwave Synthesizer at $70{ }^{\circ} \mathrm{C}$ for 30 $\mathrm{min}$. The vial was then opened, the reaction mixture was dissolved in EtOAc $(20 \mathrm{~mL})$, washed with $1 \mathrm{M}$ aqueous $\mathrm{HCl}(2 \times 20 \mathrm{~mL})$ and $10 \%(\mathrm{w} / \mathrm{v})$ aqueous $\mathrm{LiCl}(2 \times 20 \mathrm{~mL})$. The aqueous layer was then extracted with EtOAc $(3 \times 20 \mathrm{~mL})$, and the combined organic layers were dried with anhydrous $\mathrm{MgSO}_{4}$, filtered and concentrated in vacuo. The crude product was then purified by flash column chromatography, affording the pure product. 


\section{Data for Synthesised Compounds}

\section{Products from the Desulfinylative Smiles}

\section{4-nitro-N-phenylaniline (2a)}<smiles>O=[N+]([O-])c1ccc(Nc2ccccc2)cc1</smiles>

Synthesised according to general procedure B (column conditions: 0-30\% EtOAc in hexane). The pure product was afforded as a yellow solid (31 $\mathrm{mg}, 73 \%$ yield).

$1 \mathrm{mmol}$ scale procedure:

A $50 \mathrm{~mL}$ round-bottomed flask was charged with sulfinamide $3 a\left(262 \mathrm{mg}, 1 \mathrm{mmol}\right.$ ) and $\mathrm{Cs}_{2} \mathrm{CO}_{3}(1.96 \mathrm{~g}$, $6 \mathrm{mmol})$, evacuated under vacuum and filled with nitrogen. Anhydrous DMF (8 $\mathrm{mL}$ ) was added and the reaction mixture was left to stir for in a pre-warmed oil bath at $70{ }^{\circ} \mathrm{C}$ for $16 \mathrm{~h}$. the reaction mixture was dissolved in EtOAc $(30 \mathrm{~mL})$, washed with $1 \mathrm{M}$ aqueous $\mathrm{HCl}(2 \times 40 \mathrm{~mL})$ and $10 \%(\mathrm{w} / \mathrm{v})$ aqueous $\mathrm{LiCl}$ $(2 \times 40 \mathrm{~mL})$. The aqueous layer was then extracted with EtOAc $(3 \times 40 \mathrm{~mL})$, and the combined organic layers were dried with anhydrous $\mathrm{MgSO}_{4}$, filtered and concentrated in vacuo. The crude product was then purified by flash column chromatography, affording the pure product as a yellow solid $(130 \mathrm{mg}$, $61 \%$ yield).

${ }^{1} \mathrm{H}$ NMR $\left(500 \mathrm{MHz}, \mathrm{CDCl}_{3}\right) \delta 8.12(\mathrm{~d}, J=9.2 \mathrm{~Hz}, 2 \mathrm{H}), 7.39(\mathrm{t}, J=7.8 \mathrm{~Hz}, 2 \mathrm{H}), 7.21(\mathrm{~d}, J=7.9 \mathrm{~Hz}, 2 \mathrm{H}), 7.19$ $(\mathrm{t}, J=7.4 \mathrm{~Hz}, 1 \mathrm{H}), 6.94(\mathrm{~d}, J=9.2 \mathrm{~Hz}, 2 \mathrm{H}), 6.34(\mathrm{~s}, 1 \mathrm{H})$.

${ }^{13} \mathrm{C}$ NMR $\left(126 \mathrm{MHz}, \mathrm{CDCl}_{3}\right) \delta 150.2,139.8,139.5,129.8,126.3,124.7,122.0,113.7$.

HRMS (ESI) Calculated for $\mathrm{C}_{12} \mathrm{H}_{10} \mathrm{~N}_{2} \mathrm{O}_{2} \mathrm{Na}: 237.0634$. Found: $237.0627[\mathrm{M}+\mathrm{Na}]^{+}$

Data is in accordance with literature. ${ }^{3}$

2-methyl-N-(4-nitrophenyl)aniline (2b)<smiles>Cc1ccccc1Nc1ccc([N+](=O)[O-])cc1</smiles>

Synthesised according to general procedure B (column conditions: 5-30\% EtOAc in hexane). The pure product was afforded as a yellow solid ( $26 \mathrm{mg}, 56 \%$ yield).

${ }^{1} \mathrm{H}$ NMR $\left(400 \mathrm{MHz}\right.$, Acetone- $\left.\mathrm{d}_{6}\right) \delta 8.09(\mathrm{~d}, J=9.3 \mathrm{~Hz}, 2 \mathrm{H}), 8.02(\mathrm{~s}, 1 \mathrm{H}), 7.38-7.15(\mathrm{~m}, 4 \mathrm{H}), 6.85(\mathrm{~d}, J=$ $9.2 \mathrm{~Hz}, 2 \mathrm{H}), 2.26(\mathrm{~s}, 3 \mathrm{H})$.

${ }^{13} \mathrm{C}$ NMR $\left(101 \mathrm{MHz}\right.$, Acetone- $\left.\mathrm{d}_{6}\right) \delta$ 153.5, 139.4, 139.2, 134.6, 132.2, 127.9, 126.8, 126.1, 113.6, 18.0.

HRMS (APCI) Calculated for $\mathrm{C}_{13} \mathrm{H}_{13} \mathrm{~N}_{2} \mathrm{O}_{2}: 229.0972$. Found: $229.0964[\mathrm{M}+\mathrm{H}]^{+}$ 
Data is in accordance with literature. ${ }^{4}$

3-methyl-N-(4-nitrophenyl)aniline (2c)<smiles>Cc1cccc(Nc2ccc([N+](=O)[O-])cc2)c1</smiles>

Synthesised according to general procedure B (column conditions: 5-30\% EtOAc in hexane). The pure product was afforded as a yellow solid ( $27 \mathrm{mg}, 59 \%$ yield).

${ }^{1} \mathrm{H}$ NMR $\left(400 \mathrm{MHz}\right.$, Acetone-d $\left.\mathrm{d}_{6}\right) \delta 8.36(\mathrm{~s}, 1 \mathrm{H}), 8.11(\mathrm{~d}, J=9.2 \mathrm{~Hz}, 2 \mathrm{H}), 7.28(\mathrm{t}, J=7.7 \mathrm{~Hz}, 1 \mathrm{H}), 7.18-$ $7.07(\mathrm{~m}, 4 \mathrm{H}), 7.00-6.93(\mathrm{~m}, 1 \mathrm{H}), 2.34(\mathrm{~s}, 3 \mathrm{H})$.

${ }^{13} \mathrm{C}$ NMR (101 MHz, Acetone- $\left.\mathrm{d}_{6}\right)$ ) 152.0, 141.2, 140.2, 139.9, 130.2, 126.8, 125.4, 122.8, 119.3, 114.4, 21.4.

HRMS (APCI) Calculated for $\mathrm{C}_{13} \mathrm{H}_{13} \mathrm{~N}_{2} \mathrm{O}_{2}:$ 229.0972. Found: $229.0964[\mathrm{M}+\mathrm{H}]^{+}$

Data is in accordance with literature. ${ }^{4}$

\section{4-methyl-N-(4-nitrophenyl)aniline (2d)}<smiles>Cc1ccc(Nc2ccc([N+](=O)[O-])cc2)cc1</smiles>

Synthesised according to general procedure B (column conditions: 0-30\% EtOAc in hexane). The pure product was afforded as an orange solid ( $29 \mathrm{mg}, 64 \%$ yield).

${ }^{1} \mathrm{H}$ NMR $\left(400 \mathrm{MHz}, \mathrm{CDCl}_{3}\right) \delta 8.10(\mathrm{~d}, J=9.1 \mathrm{~Hz}, 2 \mathrm{H}), 7.20(\mathrm{~d}, J=8.3 \mathrm{~Hz}, 2 \mathrm{H}), 7.10(\mathrm{~d}, J=8.0 \mathrm{~Hz}, 2 \mathrm{H}), 6.87$ $(d, J=9.3 \mathrm{~Hz}, 2 \mathrm{H}), 6.29(\mathrm{~s}, 1 \mathrm{H}), 2.36(\mathrm{~s}, 3 \mathrm{H})$.

${ }^{13} \mathrm{C}$ NMR $\left(101 \mathrm{MHz}, \mathrm{CDCl}_{3}\right) \delta$ 151.0, 139.5, 136.8, 134.9, 130.4, 126.4, 122.8, 113.3, 21.1.

HRMS (ESI) Calculated for $\mathrm{C}_{13} \mathrm{H}_{11} \mathrm{~N}_{2} \mathrm{O}_{2}: 227.0821$ Found: $227.0815[\mathrm{M}-\mathrm{H}]$

Data is in accordance with literature. ${ }^{4}$

\section{4-chloro-N-(4-nitrophenyl)aniline (2e)}<smiles>O=[N+]([O-])c1ccc(Nc2ccc(Cl)cc2)cc1</smiles>

Synthesised according to general procedure B (column conditions: 0-30\% EtOAc in hexane). The pure product was afforded as an orange solid ( $25 \mathrm{mg}, 50 \%$ yield). 
${ }^{1} \mathrm{H}$ NMR $\left(500 \mathrm{MHz}, \mathrm{CDCl}_{3}\right) \delta 8.14(\mathrm{~d}, J=9.2 \mathrm{~Hz}, 2 \mathrm{H}), 7.35(\mathrm{~d}, J=8.8 \mathrm{~Hz}, 2 \mathrm{H}), 7.15(\mathrm{~d}, J=8.8 \mathrm{~Hz}, 2 \mathrm{H}), 6.92$ $(\mathrm{d}, J=9.3 \mathrm{~Hz}, 2 \mathrm{H}), 6.21(\mathrm{~s}, 1 \mathrm{H})$.

${ }^{13} \mathrm{C}$ NMR $\left(101 \mathrm{MHz}, \mathrm{CDCl}_{3}\right) \delta 149.8,140.3,138.3,130.0,129.8,126.4,123.2,114.0$.

HRMS (ESI) Calculated for $\mathrm{C}_{12} \mathrm{H}_{8} \mathrm{ClN}_{2} \mathrm{O}_{2}: 247.0280$. Found: $247.0266[\mathrm{M}-\mathrm{H}]^{-}$

Data is in accordance with literature. ${ }^{4}$

\section{4-fluoro-N-(4-nitrophenyl)aniline (2f)}<smiles>O=[N+]([O-])c1ccc(Nc2ccc(F)cc2)cc1</smiles>

Synthesised according to general procedure B (column conditions: 0-30\% EtOAc in hexane). The pure product was afforded as an orange solid ( $23 \mathrm{mg}, 50 \%$ yield).

${ }^{1} \mathrm{H}$ NMR $\left(400 \mathrm{MHz}, \mathrm{CDCl}_{3}\right) \delta 8.11(\mathrm{~d}, J=9.4 \mathrm{~Hz}, 2 \mathrm{H}), 7.22-7.16(\mathrm{~m}, 2 \mathrm{H}), 7.13-7.05(\mathrm{~m}, 2 \mathrm{H}), 6.83(\mathrm{~d}, J$ $=9.5 \mathrm{~Hz}, 2 \mathrm{H}), 6.16(\mathrm{~s}, 1 \mathrm{H})$.

${ }^{13} \mathrm{C}$ NMR $\left(101 \mathrm{MHz}, \mathrm{CDCl}_{3}\right) \delta 160.2(\mathrm{~d}, J=245.0 \mathrm{~Hz}), 150.8,139.9,135.5(\mathrm{~d}, J=2.8 \mathrm{~Hz}), 126.4,125.0$ (d, $J=8.0 \mathrm{~Hz}), 116.6(\mathrm{~d}, J=22.6 \mathrm{~Hz}), 113.3$.

${ }^{19}$ F NMR (376 MHz, $\left.\mathrm{CDCl}_{3}\right) \delta-116.63(\mathrm{~s})$.

HRMS (APCI) Calculated for $\mathrm{C}_{12} \mathrm{H}_{9} \mathrm{FN}_{2} \mathrm{O}_{2}:$ 232.0643. Found: 232.0646 [M] ${ }^{+}$

Data is in accordance with literature. ${ }^{4}$

\section{3-bromo-N-(4-nitrophenyl)aniline (2g)}<smiles>O=[N+]([O-])c1ccc(Nc2cccc(Br)c2)cc1</smiles>

Synthesised according to general procedure B (column conditions: 0-30\% EtOAc in hexane). The pure product was afforded as an orange solid ( $46 \mathrm{mg}, 78 \%$ yield).

${ }^{1} \mathrm{H}$ NMR $\left(400 \mathrm{MHz}\right.$, Acetone- $\left.\mathrm{d}_{6}\right) \delta 8.55(\mathrm{~s}, 1 \mathrm{H}), 8.15(\mathrm{~d}, J=9.2 \mathrm{~Hz}, 2 \mathrm{H}), 7.47(\mathrm{~s}, 1 \mathrm{H}), 7.37-7.31(\mathrm{~m}, 2 \mathrm{H})$, $7.29-7.24(\mathrm{~m}, 1 \mathrm{H}), 7.22(\mathrm{~d}, J=9.4 \mathrm{~Hz}, 2 \mathrm{H})$.

${ }^{13}$ C NMR (101 MHz, Acetone-d $\left.d_{6}\right) \delta 150.8,143.4,140.8,132.1,126.9,126.7,123.9,123.4,120.0,115.4$.

HRMS (APCl) Calculated for $\mathrm{C}_{12} \mathrm{H}_{10} \mathrm{BrN}_{2} \mathrm{O}_{2}: 292.9920$. Found: $292.9922[\mathrm{M}+\mathrm{H}]^{+}$

Data is in accordance with literature. ${ }^{4}$ 
(N)

Synthesised according to general procedure B (column conditions: 0-30\% EtOAc in hexane). The pure product was afforded as a yellow solid ( $32 \mathrm{mg}, 68 \%$ yield).

${ }^{1}$ H NMR $\left(400 \mathrm{MHz}\right.$, Acetone- $\left.\mathrm{d}_{6}\right) \delta 8.24(\mathrm{~s}, 1 \mathrm{H}), 8.14(\mathrm{~d}, \mathrm{~J}=9.2 \mathrm{~Hz}, 2 \mathrm{H}), 7.55-7.46(\mathrm{~m}, 1 \mathrm{H}), 7.35-7.19$ $(\mathrm{m}, 3 \mathrm{H}), 7.06(\mathrm{~d}, J=8.5 \mathrm{~Hz}, 2 \mathrm{H})$.

${ }^{13} \mathrm{C}$ NMR $\left(101 \mathrm{MHz}\right.$, Acetone- $\left.\mathrm{d}_{6}\right) \delta 156.9(\mathrm{~d}, J=245.3 \mathrm{~Hz}), 151.9,140.4,128.9(\mathrm{~d}, J=11.8 \mathrm{~Hz}), 126.7$ (d, $J=7.9 \mathrm{~Hz}), 126.6,25.9(\mathrm{~d}, J=3.7 \mathrm{~Hz}), 125.5(\mathrm{~d}, J=1.7 \mathrm{~Hz}), 117.3(\mathrm{~d}, J=19.8 \mathrm{~Hz}), 114.6$.

${ }^{19} \mathrm{~F}$ NMR $\left(376 \mathrm{MHz}\right.$, Acetone- $\left.\mathrm{d}_{6}\right) \delta-124.76(\mathrm{~s})$.

HRMS (ESI) Calculated for $\mathrm{C}_{12} \mathrm{H}_{9} \mathrm{FN}_{2} \mathrm{O}_{2} \mathrm{Na}: 255.0546$. Found: $255.0540[\mathrm{M}+\mathrm{Na}]^{+}$

\section{4-methoxy-N-(4-nitrophenyl)aniline (2i)}<smiles>COc1ccc(Nc2ccc([N+](=O)[O-])cc2)cc1</smiles>

Synthesised according to general procedure B (column conditions: 0-30\% EtOAc in hexane). The pure product was afforded as a red solid ( $25 \mathrm{mg}, 51 \%$ yield).

${ }^{1} \mathrm{H}$ NMR $\left(400 \mathrm{MHz}, \mathrm{CDCl}_{3}\right) \delta 8.08(\mathrm{~d}, J=9.2 \mathrm{~Hz}, 2 \mathrm{H}), 7.16(\mathrm{~d}, J=9.0 \mathrm{~Hz}, 2 \mathrm{H}), 6.94(\mathrm{~d}, J=8.9 \mathrm{~Hz}, 2 \mathrm{H}), 6.75$ $(\mathrm{d}, J=9.2 \mathrm{~Hz}, 2 \mathrm{H}), 6.15(\mathrm{~s}, 1 \mathrm{H}), 3.83(\mathrm{~s}, 3 \mathrm{H})$.

${ }^{13} \mathrm{C}$ NMR $\left(101 \mathrm{MHz}, \mathrm{CDCl}_{3}\right) \delta 157.6,151.8,139.2,132.1,126.5,125.7,115.1,112.8,55.7$.

HRMS (ESI) Calculated for $\mathrm{C}_{13} \mathrm{H}_{12} \mathrm{~N}_{2} \mathrm{O}_{2} \mathrm{Na:}$ 267.0740. Found: $267.0736[\mathrm{M}+\mathrm{Na}]^{+}$

Data is in accordance with literature. ${ }^{4}$

\section{3-methoxy-N-(4-nitrophenyl)aniline (2j)}<smiles>COc1cccc(Nc2ccc([N+](=O)[O-])cc2)c1</smiles>

Synthesised according to general procedure $B$, on a $0.1 \mathrm{mmol}$ scale (column conditions: 0-30\% EtOAc in hexane). The pure product was afforded as an orange solid ( $9 \mathrm{mg}, 54 \%$ yield).

${ }^{1} \mathrm{H}$ NMR $\left(500 \mathrm{MHz}, \mathrm{CDCl}_{3}\right) \delta 8.12(\mathrm{~d}, J=9.2 \mathrm{~Hz}, 2 \mathrm{H}), 7.29(\mathrm{t}, J=8.1 \mathrm{~Hz}, 1 \mathrm{H}), 6.97(\mathrm{~d}, J=9.1 \mathrm{~Hz}, 2 \mathrm{H}), 6.81$ $-6.78(\mathrm{~m}, 1 \mathrm{H}), 6.75(\mathrm{t}, J=2.3 \mathrm{~Hz}, 1 \mathrm{H}), 6.73-6.68(\mathrm{~m}, 1 \mathrm{H}), 3.82(\mathrm{~s}, 3 \mathrm{H})$.

${ }^{13} \mathrm{C}$ NMR $\left(126 \mathrm{MHz}, \mathrm{CDCl}_{3}\right) \delta 160.9,150.0,140.9,140.1,130.7,126.4,114.2,114.1,109.9,107.8,55.5$.

HRMS (ESI) Calculated for $\mathrm{C}_{13} \mathrm{H}_{12} \mathrm{~N}_{2} \mathrm{O}_{2} \mathrm{Na:}$ 267.0740. Found: $267.0736[\mathrm{M}+\mathrm{Na}]^{+}$ 
Data is in accordance with literature. ${ }^{4}$

\section{3,5-dimethoxy-N-(4-nitrophenyl)aniline (2k)}<smiles>COc1cc(Nc2ccc([N+](=O)[O-])cc2)cc(OC)c1</smiles>

Synthesised according to general procedure B (column conditions: 0-30\% EtOAc in hexane). The pure product was afforded as a red solid ( $33 \mathrm{mg}, 61 \%$ yield).

${ }^{1} \mathrm{H}$ NMR $\left(500 \mathrm{MHz}, \mathrm{DMSO}-\mathrm{d}_{6}\right) \delta 9.27(\mathrm{~s}, 1 \mathrm{H}), 8.09(\mathrm{~d}, J=9.5 \mathrm{~Hz}, 2 \mathrm{H}), 7.10(\mathrm{~d}, J=9.5 \mathrm{~Hz}, 2 \mathrm{H}), 6.37(\mathrm{~d}, J$ $=2.2 \mathrm{~Hz}, 2 \mathrm{H}), 6.25(\mathrm{t}, \mathrm{J}=2.2 \mathrm{~Hz}, 1 \mathrm{H}), 3.74(\mathrm{~s}, 6 \mathrm{H})$.

${ }^{13} \mathrm{C}$ NMR $\left(126 \mathrm{MHz}, \mathrm{DMSO}-\mathrm{d}_{6}\right) \delta$ 161.2, 150.5, 141.9, 138.1, 126.1, 114.0, 98.7, 95.3, 55.2.

HRMS (APCl) Calculated for $\mathrm{C}_{14} \mathrm{H}_{15} \mathrm{~N}_{2} \mathrm{O}_{4}: 275.1026$. Found: $275.1015[\mathrm{M}+\mathrm{H}]^{+}$

MP $150-152^{\circ} \mathrm{C}$

\section{4-nitro-N-(4-(trifluoromethyl)phenyl)aniline (2I)}<smiles>O=[N+]([O-])c1ccc(Nc2ccc(C(F)(F)F)cc2)cc1</smiles>

Synthesised according to general procedure B (column conditions: 0-30\% EtOAc in hexane). The pure product was afforded as a yellow solid (40 $\mathrm{mg}, 70 \%$ yield).

${ }^{1} \mathrm{H}$ NMR $\left(400 \mathrm{MHz}\right.$, Acetone- $\left.\mathrm{d}_{6}\right) \delta 8.76(\mathrm{~s}, 1 \mathrm{H}), 8.18(\mathrm{~d}, J=8.8 \mathrm{~Hz}, 2 \mathrm{H}), 7.70(\mathrm{~d}, J=8.6 \mathrm{~Hz}, 2 \mathrm{H}), 7.48(\mathrm{~d}$, $J=8.3 \mathrm{~Hz}, 2 \mathrm{H}), 7.33(\mathrm{~d}, J=9.2 \mathrm{~Hz}, 2 \mathrm{H})$.

${ }^{13} \mathrm{C}$ NMR (101 MHz, Acetone- $\left.\mathrm{d}_{6}\right) \delta$ 150.0, 145.5, 141.4, 127.6 (q, $\left.J=3.8 \mathrm{~Hz}\right), 126.6,124.3$ (q, $J=32.6$ $\mathrm{Hz}), 121.5,120.0,116.3$.

${ }^{19}$ F NMR (376 MHz, Acetone- $d_{6}$ ) $\delta-62.28$ (s).

HRMS (APCI) Calculated for $\mathrm{C}_{13} \mathrm{H}_{10} \mathrm{~F}_{3} \mathrm{~N}_{2} \mathrm{O}_{2}$ : 283.0689. Found: $283.0686[\mathrm{M}+\mathrm{H}]^{+}$

Data is in accordance with literature. ${ }^{5}$

\section{$\mathrm{N}$-(4-nitrophenyl)naphthalen-1-amine (2m)}<smiles>O=[N+]([O-])c1ccc(Nc2cccc3ccccc23)cc1</smiles> 
Synthesised according to general procedure B (column conditions: 0-25\% EtOAc in hexane). The pure product was afforded as a brown solid ( $33 \mathrm{mg}, 61 \%$ yield).

${ }^{1} \mathrm{H}$ NMR $\left(400 \mathrm{MHz}\right.$, Acetone- $\left.\mathrm{d}_{6}\right) \delta 9.41(\mathrm{~s}, 1 \mathrm{H}), 8.06(\mathrm{~d}, J=9.0 \mathrm{~Hz}, 2 \mathrm{H}), 8.02-7.96(\mathrm{~m}, 2 \mathrm{H}), 7.84(\mathrm{~d}, J=$ $7.9 \mathrm{~Hz}, 1 \mathrm{H}), 7.62-7.48(\mathrm{~m}, 4 \mathrm{H}), 6.89(\mathrm{~d}, J=9.4 \mathrm{~Hz}, 2 \mathrm{H})$.

${ }^{13}$ C NMR (101 MHz, Acetone-d $\left.d_{6}\right) \delta 153.0,137.6,135.6,134.4,128.8,128.4,126.5,126.3,126.2,126.1$, $125.7,122.8,121.3,113.0$.

HRMS (ESI) Calculated for $\mathrm{C}_{16} \mathrm{H}_{11} \mathrm{~N}_{2} \mathrm{O}_{2}: 263.0815$. Found: $263.0812[\mathrm{M}-\mathrm{H}]^{-}$

Data is in accordance with literature. ${ }^{4}$

$\mathrm{N}$-(4-nitrophenyl)pyridin-2-amine (2n)<smiles>O=[N+]([O-])c1ccc(Nc2ccccn2)cc1</smiles>

Synthesised according to general procedure B (column conditions: 0-50\% EtOAc in hexane). The pure product was afforded as an orange solid ( $17 \mathrm{mg}, 40 \%$ yield).

${ }^{1} \mathrm{H}$ NMR $\left(500 \mathrm{MHz}\right.$, Acetone- $\left.\mathrm{d}_{6}\right) \delta 9.12(\mathrm{~s}, 1 \mathrm{H}), 8.36-8.31(\mathrm{~m}, 1 \mathrm{H}), 8.18(\mathrm{~d}, J=9.4 \mathrm{~Hz}, 2 \mathrm{H}), 8.01(\mathrm{~d}, J=$ $9.3 \mathrm{~Hz}, 2 \mathrm{H}), 7.69(\mathrm{t}, J=8.7 \mathrm{~Hz}, 1 \mathrm{H}), 7.01(\mathrm{~d}, J=8.3 \mathrm{~Hz}, 1 \mathrm{H}), 6.97-6.93(\mathrm{~m}, 1 \mathrm{H})$.

${ }^{13} \mathrm{C}$ NMR $\left(126 \mathrm{MHz}\right.$, Acetone- $\left.\mathrm{d}_{6}\right) \delta$ 154.9, 148.2, 147.4, 140.3, 137.8, 125.0, 116.7, 116.6, 112.2.

HRMS (ESI) Calculated for $\mathrm{C}_{13} \mathrm{H}_{9} \mathrm{~N}_{3} \mathrm{O}_{2} \mathrm{Na}$ : 238.0587. Found: $238.0581[\mathrm{M}+\mathrm{Na}]^{+}$

Data is in accordance with literature. ${ }^{6}$

$\mathrm{N}$-(4-nitrophenyl)quinolin-8-amine (20)<smiles>O=[N+]([O-])c1ccc(Nc2cccc3cccnc23)cc1</smiles>

Synthesised according to general procedure B (column conditions: 0-50\% EtOAc in hexane). The pure product was afforded as an orange solid ( $26 \mathrm{mg}, 50 \%$ yield).

${ }^{1} \mathrm{H}$ NMR $\left(400 \mathrm{MHz}\right.$, Acetone- $\left.\mathrm{d}_{6}\right) \delta 9.19(\mathrm{~s}, 1 \mathrm{H}), 8.90(\mathrm{dd}, J=4.2,1.7 \mathrm{~Hz}, 1 \mathrm{H}), 8.38(\mathrm{dd}, J=8.3,1.7 \mathrm{~Hz}$, $1 \mathrm{H}), 8.25(\mathrm{~d}, J=9.1 \mathrm{~Hz}, 2 \mathrm{H}), 7.87(\mathrm{dd}, J=5.9,2.9 \mathrm{~Hz}, 1 \mathrm{H}), 7.69-7.55(\mathrm{~m}, 5 \mathrm{H})$.

${ }^{13}$ C NMR (101 MHz, Acetone- $\left.\mathrm{d}_{6}\right) \delta$ 150.0, 149.4, 140.4, 138.1, 137.4, 129.9, 127.8, 126.6, 123.2, 121.3, $116.9,116.9,113.8$.

HRMS (ESI) Calculated for $\mathrm{C}_{15} \mathrm{H}_{11} \mathrm{~N}_{3} \mathrm{O}_{2} \mathrm{Na:} 288.0743$. Found: $288.0731[\mathrm{M}+\mathrm{Na}]^{+}$

Data is in accordance with literature. ${ }^{4}$ 
<smiles>Cc1cc(C)c(Nc2ccc([N+](=O)[O-])cc2)c(C)c1</smiles>

Synthesised according to general procedure B (column conditions: 0-25\% EtOAc in hexane). The pure product was afforded as a yellow solid ( $37 \mathrm{mg}, 71 \%$ yield).

${ }^{1} \mathrm{H}$ NMR $\left(500 \mathrm{MHz}, \mathrm{CDCl}_{3}\right) \delta 8.06(\mathrm{~d}, J=8.7 \mathrm{~Hz}, 2 \mathrm{H}), 6.97(\mathrm{~s}, 2 \mathrm{H}), 6.44(\mathrm{~d}, J=8.7 \mathrm{~Hz}, 2 \mathrm{H}), 5.86(\mathrm{~s}, 1 \mathrm{H})$, $2.32(\mathrm{~s}, 3 \mathrm{H}), 2.16(\mathrm{~s}, 6 \mathrm{H})$.

${ }^{13} \mathrm{C}$ NMR $\left(126 \mathrm{MHz}, \mathrm{CDCl}_{3}\right) \delta 152.6,138.7,137.5,136.5,133.1,129.6,126.6,111.7,21.1,18.2$.

HRMS (APCI) Calculated for $\mathrm{C}_{15} \mathrm{H}_{17} \mathrm{~N}_{2} \mathrm{O}_{2}: 257.1285$. Found: $257.1272[\mathrm{M}+\mathrm{H}]^{+}$

MP $125-127^{\circ} \mathrm{C}$

\section{2,6-diisopropyl-N-(4-nitrophenyl)aniline (2q)}<smiles>CC(C)c1cccc(C(C)C)c1Nc1ccc([N+](=O)[O-])cc1</smiles>

Synthesised according to general procedure B (column conditions: 0-25\% EtOAc in hexane). The pure product was afforded as a yellow solid ( $31 \mathrm{mg}, 51 \%$ yield).

${ }^{1} \mathrm{H}$ NMR (400 MHz, DMSO-d 6 ) $\delta 8.76(\mathrm{~s}, 1 \mathrm{H}), 7.99(\mathrm{~d}, J=34.0 \mathrm{~Hz}, 2 \mathrm{H}), 7.39-7.32(\mathrm{~m}, 1 \mathrm{H}), 7.27(\mathrm{~d}, J=$ $7.1 \mathrm{~Hz}, 2 \mathrm{H}), 6.99(\mathrm{~s}, 1 \mathrm{H}), 5.90(\mathrm{~s}, 1 \mathrm{H}), 3.00(\mathrm{~h}, J=6.9 \mathrm{~Hz}, 2 \mathrm{H}), 1.13(\mathrm{~d}, J=6.8 \mathrm{~Hz}, 6 \mathrm{H}), 1.05(\mathrm{~d}, J=6.9 \mathrm{~Hz}$, $6 \mathrm{H})$.

${ }^{13} \mathrm{C}$ NMR $\left(101 \mathrm{MHz}, \mathrm{DMSO}-\mathrm{d}_{6}\right) \delta 154.8,146.9,136.4,133.2,128.2,126.8,124.0,113.2,108.8,27.9$, 24.1, 23.0.

HRMS (APCI) Calculated for $\mathrm{C}_{20} \mathrm{H}_{25} \mathrm{~N}_{2} \mathrm{O}_{2}:$ 297.1603. Found: 297.1609 [M-H]

MP $140-142{ }^{\circ} \mathrm{C}$

\section{2-(tert-butyl)-N-(4-nitrophenyl)aniline (2r)}<smiles>CC(C)(C)c1ccccc1Nc1ccc([N+](=O)[O-])cc1</smiles>

Synthesised according to general procedure B (column conditions: 0-30\% EtOAc in hexane). The pure product was afforded as an orange solid ( $38 \mathrm{mg}, 69 \%$ yield).

${ }^{1} \mathrm{H}$ NMR $\left(500 \mathrm{MHz}, \mathrm{DMSO}-\mathrm{d}_{6}\right) \delta 8.64(\mathrm{~s}, 1 \mathrm{H}), 8.01(\mathrm{~d}, J=9.3 \mathrm{~Hz}, 2 \mathrm{H}), 7.55-7.48(\mathrm{~m}, 1 \mathrm{H}), 7.34-7.27$ $(\mathrm{m}, 2 \mathrm{H}), 7.16-7.09(\mathrm{~m}, 1 \mathrm{H}), 6.58(\mathrm{~d}, J=9.3 \mathrm{~Hz}, 2 \mathrm{H}), 1.31(\mathrm{~s}, 9 \mathrm{H})$. 
${ }^{13}$ C NMR (126 MHz, DMSO-d 6 ) $\delta$ 154.6, 147.7, 138.1, 136.6, 130.9, 127.6, 127.5, 127.4, 126.2, 112.3, 34.9, 30.6 .

HRMS (APCI) Calculated for $\mathrm{C}_{16} \mathrm{H}_{19} \mathrm{~N}_{2} \mathrm{O}_{2}: 271.1441$. Found: $271.1435[\mathrm{M}+\mathrm{H}]^{+}$

MP $91-94^{\circ} \mathrm{C}$

2-methoxy-4-nitro-N-phenylaniline (2s)<smiles>COc1cc([N+](=O)[O-])ccc1Nc1ccccc1</smiles>

Synthesised according to general procedure B (column conditions: 0-30\% EtOAc in hexane). The pure product was afforded as a yellow solid ( $32 \mathrm{mg}, 65 \%$ yield).

${ }^{1} \mathrm{H}$ NMR $\left(400 \mathrm{MHz}, \mathrm{CDCl}_{3}\right) \delta 7.80-7.70(\mathrm{~m}, 1 \mathrm{H}), 7.66(\mathrm{~s}, 1 \mathrm{H}), 7.31(\mathrm{t}, J=8.1 \mathrm{~Hz}, 2 \mathrm{H}), 7.21-7.13(\mathrm{~m}$, $2 \mathrm{H}), 7.12-7.06(\mathrm{~m}, 1 \mathrm{H}), 7.04(\mathrm{~d}, J=9.0 \mathrm{~Hz}, 1 \mathrm{H}), 3.93(\mathrm{~s}, 3 \mathrm{H})$.

${ }^{13} \mathrm{C}$ NMR $\left(101 \mathrm{MHz}, \mathrm{CDCl}_{3}\right) \delta$ 146.0, 140.8, 139.5, 139.0, 129.8, 124.6, 122.2, 119.2, 109.5, 105.7, 56.3.

HRMS (APCI) Calculated for $\mathrm{C}_{13} \mathrm{H}_{13} \mathrm{~N}_{2} \mathrm{O}_{2}:$ 245.0921. Found: $245.0914[\mathrm{M}+\mathrm{H}]^{+}$

Data is in accordance with literature. ${ }^{7}$

\section{5-nitro-N-phenylpyridin-2-amine (2t)}<smiles>O=[N+]([O-])c1ccc(Nc2ccccc2)nc1</smiles>

Synthesised according to general procedure B, $0.1 \mathrm{mmol}$ scale (column conditions: $0-40 \%$ EtOAc in hexane). The pure product was afforded as a yellow solid (14 $\mathrm{mg}, 63 \%$ yield).

${ }^{1} \mathrm{H}$ NMR $\left(500 \mathrm{MHz}\right.$, Acetone- $\left.\mathrm{d}_{6}\right) \delta 9.07(\mathrm{~d}, J=2.7 \mathrm{~Hz}, 1 \mathrm{H}), 8.31(\mathrm{dd}, J=9.3,2.8 \mathrm{~Hz}, 1 \mathrm{H}), 7.76(\mathrm{~d}, J=8.1$ $\mathrm{Hz}, 2 \mathrm{H}), 7.39(\mathrm{t}, J=7.6 \mathrm{~Hz}, 2 \mathrm{H}), 7.13(\mathrm{t}, J=7.4 \mathrm{~Hz}, 1 \mathrm{H}), 6.99(\mathrm{~d}, J=9.3 \mathrm{~Hz}, 1 \mathrm{H})$.

${ }^{13} \mathrm{C}$ NMR (126 MHz, Acetone- $\left.\mathrm{d}_{6}\right) \delta$ 160.0, 146.3, 140.3, 137.7, 133.5, 129.8, 124.6, 121.5, 110.4.

HRMS (APCI) Calculated for $\mathrm{C}_{11} \mathrm{H}_{10} \mathrm{~N}_{3} \mathrm{O}_{2}: 216.0768$. Found: $216.0761[\mathrm{M}+\mathrm{H}]^{+}$

Data is in accordance with literature. ${ }^{8}$ 


\section{Sulfinamide Starting Materials}

4-nitro-N-phenylbenzenesulfinamide (3a)<smiles>O=[N+]([O-])c1ccc(S(=O)Nc2ccccc2)cc1</smiles>

Prepared according to general procedure A, $4 \mathrm{mmol}$ scale (column conditions: $0-40 \%$ EtOAc in hexane). The pure product was afforded as a yellow solid ( $519 \mathrm{mg}, 49 \%$ yield).

${ }^{1} \mathrm{H}$ NMR $(500 \mathrm{MHz}$, DMSO-d 6 ) $\delta 9.59(\mathrm{~s}, 1 \mathrm{H}), 8.40(\mathrm{~d}, J=9.1 \mathrm{~Hz}, 2 \mathrm{H}), 7.98(\mathrm{~d}, J=9.1 \mathrm{~Hz}, 2 \mathrm{H}), 7.25(\mathrm{t}, J=$ $7.7 \mathrm{~Hz}, 2 \mathrm{H}), 7.07(\mathrm{~d}, J=7.4 \mathrm{~Hz}, 2 \mathrm{H}), 6.97(\mathrm{t}, J=7.4 \mathrm{~Hz}, 1 \mathrm{H})$.

${ }^{13} \mathrm{C}$ NMR (126 MHz, DMSO-d 6 ) $\delta$ 151.5, 149.1, 141.2, 129.4, 127.4, 124.2, 122.7, 118.2.

HRMS (ESI) Calculated for $\mathrm{C}_{12} \mathrm{H}_{9} \mathrm{~N}_{2} \mathrm{O}_{3} \mathrm{~S}: 261.0339$. Found: 261.0334 [M-H]

Data is in accordance with literature. ${ }^{9}$

\section{4-nitro-N-(o-tolyl)benzenesulfinamide (3b)}<smiles>Cc1ccccc1NS(=O)c1ccc([N+](=O)[O-])cc1</smiles>

Prepared according to general procedure A (column conditions: 5-40\% EtoAc in hexane). The pure product was afforded as an orange solid ( $83 \mathrm{mg}, 30 \%$ yield).

${ }^{1} \mathrm{H}$ NMR $\left(500 \mathrm{MHz}\right.$, Acetone- $\left.\mathrm{d}_{6}\right) \delta 8.40(\mathrm{~d}, J=9.0 \mathrm{~Hz}, 2 \mathrm{H}), 8.12-8.03(\mathrm{~m}, 3 \mathrm{H}), 7.39-7.33(\mathrm{~m}, 1 \mathrm{H}), 7.19$ $(\mathrm{d}, J=7.2 \mathrm{~Hz}, 1 \mathrm{H}), 7.11(\mathrm{t}, J=7.7 \mathrm{~Hz}, 1 \mathrm{H}), 7.01(\mathrm{t}, J=7.5 \mathrm{~Hz}, 1 \mathrm{H}), 2.32(\mathrm{~s}, 3 \mathrm{H})$.

${ }^{13}$ C NMR (126 MHz, Acetone-d 6 ) $\delta$ 153.2, 150.5, 140.1, 131.7, 131.0, 128.3, 127.5, 125.3, 124.7, 122.6, 18.2.

HRMS (APCI) Calculated for $\mathrm{C}_{13} \mathrm{H}_{13} \mathrm{~N}_{2} \mathrm{O}_{3} \mathrm{~S}: 277.0641$. Found: $277.0631[\mathrm{M}+\mathrm{H}]^{+}$

MP $120-123^{\circ} \mathrm{C}$

\section{4-nitro-N-(m-tolyl)benzenesulfinamide (3c)}<smiles>Cc1cccc(NS(=O)c2ccc([N+](=O)[O-])cc2)c1</smiles>

Prepared according to general procedure A (column conditions: 5-40\% EtOAc in hexane). The pure product was afforded as a yellow solid ( $202 \mathrm{mg}, 73 \%$ yield). 
1H NMR (500 MHz, Acetone- $\left.\mathrm{d}_{6}\right) \delta 8.45-8.39(\mathrm{~m}, 3 \mathrm{H}), 8.07(\mathrm{~d}, \mathrm{~J}=8.2 \mathrm{~Hz}, 2 \mathrm{H}), 7.15(\mathrm{t}, \mathrm{J}=7.8 \mathrm{~Hz}, 1 \mathrm{H})$, $7.02(\mathrm{~s}, 1 \mathrm{H}), 6.97(\mathrm{~d}, \mathrm{~J}=8.5 \mathrm{~Hz}, 1 \mathrm{H}), 6.86(\mathrm{~d}, \mathrm{~J}=7.5 \mathrm{~Hz}, 1 \mathrm{H}), 2.28(\mathrm{~s}, 3 \mathrm{H})$.

${ }^{13} \mathrm{C}$ NMR (126 MHz, Acetone- $\mathrm{d}_{6}$ ) $\delta$ 153.1, 150.5, 142.2, 140.0, 130.0, 128.2, 124.8, 124.8, 120.2, 116.8, 21.4.

HRMS (APCI) Calculated for $\mathrm{C}_{13} \mathrm{H}_{13} \mathrm{~N}_{2} \mathrm{O}_{3} \mathrm{~S}: 277.0641$. Found: $277.0634[\mathrm{M}+\mathrm{H}]^{+}$

MP $119-121^{\circ} \mathrm{C}$

4-nitro-N-(p-tolyl)benzenesulfinamide (3d)<smiles>Cc1ccc(NS(=O)c2ccc([N+](=O)[O-])cc2)cc1</smiles>

Synthesised according to general procedure A (column conditions: 5-40\% EtOAc in hexane). The pure product was afforded as a yellow solid ( $185 \mathrm{mg}, 67 \%$ yield).

${ }^{1} \mathrm{H}$ NMR (400 MHz, Acetone- $\left.\mathrm{d}_{6}\right) \delta 8.39(\mathrm{~d}, J=8.8 \mathrm{~Hz}, 2 \mathrm{H}), 8.37(\mathrm{~s}, 1 \mathrm{H}), 8.06(\mathrm{~d}, J=8.8 \mathrm{~Hz}, 2 \mathrm{H}), 7.12-$ $7.02(\mathrm{~m}, 4 \mathrm{H}), 2.25(\mathrm{~s}, 3 \mathrm{H})$.

${ }^{13}$ C NMR (101 MHz, Acetone- $\left.d_{6}\right) \delta$ 153.2, 150.5, 139.4, 133.7, 130.6, 128.2, 124.8, 120.5, 20.7.

HRMS (ESI) Calculated for $\mathrm{C}_{13} \mathrm{H}_{11} \mathrm{~N}_{2} \mathrm{O}_{3} \mathrm{~S}: 275.0496$. Found: 275.0487 [M-H]

Data is in accordance with literature. ${ }^{10}$

N-(4-chlorophenyl)-4-nitrobenzenesulfinamide (3e)<smiles>O=[N+]([O-])c1ccc(S(=O)Nc2ccc(Cl)cc2)cc1</smiles>

Synthesised according to general procedure A (column conditions: 5-40\% EtOAc in hexane). The pure product was afforded as a red solid ( $85 \mathrm{mg}, 29 \%$ yield).

${ }^{1} \mathrm{H}$ NMR $\left(400 \mathrm{MHz}\right.$, Acetone- $\left.\mathrm{d}_{6}\right) \delta 8.66(\mathrm{~s}, 1 \mathrm{H}), 8.43(\mathrm{~d}, J=9.1 \mathrm{~Hz}, 2 \mathrm{H}), 8.07(\mathrm{~d}, J=9.1 \mathrm{~Hz}, 2 \mathrm{H}), 7.29$ (d, $J=9.1 \mathrm{~Hz}, 2 \mathrm{H}), 7.19(\mathrm{~d}, J=8.9 \mathrm{~Hz}, 2 \mathrm{H})$.

${ }^{13} \mathrm{C}$ NMR $\left(101 \mathrm{MHz}\right.$, Acetone- $\left.\mathrm{d}_{6}\right) \delta 152.6,150.6,141.0,130.1,128.7,128.2,124.9,121.4$.

HRMS (ESI) Calculated for $\mathrm{C}_{12} \mathrm{H}_{8} \mathrm{ClN}_{2} \mathrm{O}_{3} \mathrm{~S}: 294.9950$. Found: 294.9942 [M-H]

MP $154-156^{\circ} \mathrm{C}$ 
<smiles>O=[N+]([O-])c1ccc(S(=O)Nc2ccc(F)cc2)cc1</smiles>

Synthesised according to general procedure A (column conditions: 5-40\% EtOAc in hexane). The pure product was afforded as a yellow solid ( $144 \mathrm{mg}, 52 \%$ yield).

${ }^{1} \mathrm{H}$ NMR $\left(400 \mathrm{MHz}\right.$, Acetone- $\left.\mathrm{d}_{6}\right) \delta 8.51(\mathrm{~s}, 1 \mathrm{H}), 8.41(\mathrm{~d}, J=8.9 \mathrm{~Hz}, 2 \mathrm{H}), 8.06(\mathrm{~d}, J=8.9 \mathrm{~Hz}, 2 \mathrm{H}), 7.25-$ $7.15(\mathrm{~m}, 2 \mathrm{H}), 7.11-7.00(\mathrm{~m}, 2 \mathrm{H})$.

${ }^{13} \mathrm{C}$ NMR (101 MHz, Acetone- $\left.\mathrm{d}_{6}\right) \delta 159.2(\mathrm{~d}, J=240.2 \mathrm{~Hz}), 151.8,149.6,137.0,127.3,123.9,121.8$ (d, $J=8.2 \mathrm{~Hz}), 115.7(\mathrm{~d}, J=22.9 \mathrm{~Hz})$.

${ }^{19}$ F NMR (376 MHz, Acetone- $\left.d_{6}\right) \delta-121.16(s)$.

HRMS (APCI) Calculated for $\mathrm{C}_{12} \mathrm{H}_{10} \mathrm{FN}_{2} \mathrm{O}_{3} \mathrm{~S}: 281.0391$. Found: $281.0383[\mathrm{M}+\mathrm{H}]^{+}$

MP $132-134^{\circ} \mathrm{C}$

\section{N-(3-bromophenyl)-4-nitrobenzenesulfinamide (3g)}<smiles>O=[N+]([O-])c1ccc(S(=O)Nc2cccc(Br)c2)cc1</smiles>

Synthesised according to general procedure A (column conditions: 5-40\% EtOAc in hexane). The pure product was afforded as a yellow solid ( $241 \mathrm{mg}, 71 \%$ yield).

${ }^{1} \mathrm{H}$ NMR $(500 \mathrm{MHz}$, DMSO-d $) \delta 9.81(\mathrm{~s}, 1 \mathrm{H}), 8.41(\mathrm{~d}, J=8.5 \mathrm{~Hz}, 2 \mathrm{H}), 8.00(\mathrm{~d}, J=8.9 \mathrm{~Hz}, 2 \mathrm{H}), 7.25(\mathrm{t}, J=$ $2.0 \mathrm{~Hz}, 1 \mathrm{H}), 7.21(\mathrm{t}, J=7.9 \mathrm{~Hz}, 1 \mathrm{H}), 7.18-7.13(\mathrm{~m}, 1 \mathrm{H}), 7.11-7.05(\mathrm{~m}, 1 \mathrm{H})$.

${ }^{13}$ C NMR (126 MHz, DMSO-d 6 ) $\delta$ 151.0, 149.3, 143.1, 131.4, 127.4, 125.2, 124.2, 122.1, 120.3, 116.8.

HRMS (APCI) Calculated for $\mathrm{C}_{12} \mathrm{H}_{8} \mathrm{BrN}_{2} \mathrm{O}_{3} \mathrm{~S}: 338.9444$. Found: $338.9442[\mathrm{M}-\mathrm{H}]$

MP $108-109^{\circ} \mathrm{C}$

\section{$\mathrm{N}$-(2-fluorophenyl)-4-nitrobenzenesulfinamide (3h)}<smiles>O=[N+]([O-])c1ccc(S(=O)Nc2ccccc2F)cc1</smiles>

Synthesised according to general procedure A (column conditions: 5-40\% EtOAc in hexane). The pure product was afforded as an off-white solid ( $189 \mathrm{mg}, 68 \%$ yield). 
${ }^{1} \mathrm{H}$ NMR $\left(500 \mathrm{MHz}, \mathrm{DMSO}-\mathrm{d}_{6}\right) \delta 9.59(\mathrm{~s}, 1 \mathrm{H}), 8.39(\mathrm{~d}, J=9.1 \mathrm{~Hz}, 2 \mathrm{H}), 7.95(\mathrm{~d}, J=8.8 \mathrm{~Hz}, 2 \mathrm{H}), 7.31-7.15$ $(\mathrm{m}, 2 \mathrm{H}), 7.09-7.00(\mathrm{~m}, 2 \mathrm{H})$.

${ }^{13} \mathrm{C}$ NMR (126 MHz, DMSO-d $\left.\mathrm{d}_{6}\right) \delta 153.7(\mathrm{~d}, J=243.6 \mathrm{~Hz}), 151.2,149.1,128.2(\mathrm{~d}, J=12.6 \mathrm{~Hz}), 127.4$, $124.8-124.6(\mathrm{~m}), 124.1,122.2(\mathrm{~d}, J=1.9 \mathrm{~Hz}), 116.0(\mathrm{~d}, J=19.2 \mathrm{~Hz})$.

${ }^{19}$ F NMR (471 MHz, DMSO-d 6 ) $\delta-126.15--126.23(\mathrm{~m})$.

HRMS (APCl) Calculated for $\mathrm{C}_{12} \mathrm{H}_{10} \mathrm{FN}_{2} \mathrm{O}_{3} \mathrm{~S}: 281.0391$. Found: $281.0387[\mathrm{M}+\mathrm{H}]^{+}$

MP $142-144^{\circ} \mathrm{C}$

N-(4-methoxyphenyl)-4-nitrobenzenesulfinamide (3i)<smiles>COc1ccc(NS(=O)c2ccc([N+](=O)[O-])cc2)cc1</smiles>

Synthesised according to general procedure A (column conditions: 5-40\% EtOAc in hexane). The pure product was afforded as a bronze solid (161 $\mathrm{mg}, 55 \%$ yield).

${ }^{1}$ H NMR (400 MHz, Acetone- $\left.d_{6}\right) \delta 8.39(\mathrm{~d}, J=9.1 \mathrm{~Hz}, 2 \mathrm{H}), 8.20(\mathrm{~s}, 1 \mathrm{H}), 8.03(\mathrm{~d}, J=9.1 \mathrm{~Hz}, 2 \mathrm{H}), 7.09$ (d, $J=9.0 \mathrm{~Hz}, 2 \mathrm{H}), 6.84(\mathrm{~d}, J=8.5 \mathrm{~Hz}, 2 \mathrm{H}), 3.74(\mathrm{~s}, 3 \mathrm{H})$.

${ }^{13} \mathrm{C}$ NMR $\left(101 \mathrm{MHz}\right.$, Acetone- $\left.\mathrm{d}_{6}\right) \delta$ 157.6, 153.2, 150.4, 134.2, 128.3, 124.7, 123.6, 115.2, 55.7.

HRMS (ESI) Calculated for $\mathrm{C}_{13} \mathrm{H}_{11} \mathrm{~N}_{2} \mathrm{O}_{4} \mathrm{~S}: 291.0445$. Found: 291.0435 [M-H]

MP $162-163^{\circ} \mathrm{C}$

N-(3-methoxyphenyl)-4-nitrobenzenesulfinamide (3j)<smiles>COc1cccc(NS(=O)c2ccc([N+](=O)[O-])cc2)c1</smiles>

Synthesised according to general procedure A (column conditions: 0-40\% EtOAc in hexane). The pure product was afforded as a brown solid ( $37 \mathrm{mg}, 13 \%$ yield).

${ }^{1} \mathrm{H}$ NMR $\left(400 \mathrm{MHz}\right.$, Acetone- $\left.\mathrm{d}_{6}\right) \delta 8.52(\mathrm{~s}, 1 \mathrm{H}), 8.43(\mathrm{~d}, J=8.9 \mathrm{~Hz}, 2 \mathrm{H}), 8.08(\mathrm{~d}, J=8.9 \mathrm{~Hz}, 2 \mathrm{H}), 7.23-$ $7.14(\mathrm{~m}, 1 \mathrm{H}), 6.80-6.73(\mathrm{~m}, 2 \mathrm{H}), 6.64-6.57(\mathrm{~m}, 1 \mathrm{H}), 3.75(\mathrm{~s}, 3 \mathrm{H})$.

${ }^{13} \mathrm{C}$ NMR (101 MHz, Acetone- $\left.\mathrm{d}_{6}\right) \delta$ 161.6, 53.1, 143.5, 131.0, 128.2, 126.2, 124.8, 111.8, 109.4, 105.5, 55.5.

HRMS (ESI) Calculated for $\mathrm{C}_{13} \mathrm{H}_{11} \mathrm{~N}_{2} \mathrm{O}_{4} \mathrm{~S}: 291.0445$. Found: 291.0435 [M-H]

MP $100-102^{\circ} \mathrm{C}$ 
<smiles>COc1cc(NS(=O)c2ccc([N+](=O)[O-])cc2)cc(OC)c1</smiles>

Synthesised according to general procedure A (column conditions: 0-50\% EtOAc in hexane). The pure product was afforded as an orange solid (170 $\mathrm{mg}, 53 \%$ yield).

${ }^{1} \mathrm{H}$ NMR $\left(400 \mathrm{MHz}, \mathrm{DMSO}-\mathrm{d}_{6}\right) \delta 9.56(\mathrm{~s}, 1 \mathrm{H}), 8.42(\mathrm{~d}, J=8.9 \mathrm{~Hz}, 2 \mathrm{H}), 7.98(\mathrm{~d}, J=8.7 \mathrm{~Hz}, 2 \mathrm{H}), 6.23(\mathrm{~d}, J$ $=2.2 \mathrm{~Hz}, 2 \mathrm{H}), 6.13(\mathrm{t}, J=2.2 \mathrm{~Hz}, 1 \mathrm{H}), 3.67(\mathrm{~s}, 6 \mathrm{H})$.

${ }^{13}$ C NMR (101 MHz, DMSO-d 6 ) $\delta$ 161.0, 151.4, 149.1, 143.1, 127.3, 124.2, 96.2, 94.4, 55.1.

HRMS (APCI) Calculated for $\mathrm{C}_{14} \mathrm{H}_{15} \mathrm{~N}_{2} \mathrm{O}_{5} \mathrm{~S}: 323.0696$. Found: $323.0688[\mathrm{M}+\mathrm{H}]^{+}$

MP $130-132^{\circ} \mathrm{C}$

4-nitro-N-(4-(trifluoromethyl)phenyl)benzenesulfinamide (3l)<smiles>O=[N+]([O-])c1ccc(S(=O)Nc2ccc(C(F)(F)F)cc2)cc1</smiles>

Synthesised according to general procedure A (column conditions: 5-40\% EtOAc in hexane). The pure product was afforded as a yellow solid ( $204 \mathrm{mg}, 62 \%$ yield).

${ }^{1} \mathrm{H}$ NMR $\left(400 \mathrm{MHz}\right.$, Acetone- $\left.\mathrm{d}_{6}\right) \delta 9.03(\mathrm{~s}, 1 \mathrm{H}), 8.44(\mathrm{~d}, J=9.1 \mathrm{~Hz}, 2 \mathrm{H}), 8.11(\mathrm{~d}, J=8.7 \mathrm{~Hz}, 2 \mathrm{H}), 7.64(\mathrm{~d}$, $J=8.3 \mathrm{~Hz}, 2 \mathrm{H}), 7.37(\mathrm{~d}, J=8.4 \mathrm{~Hz}, 2 \mathrm{H})$.

${ }^{13} \mathrm{C}$ NMR (101 MHz, Acetone- $\mathrm{d}_{6}$ ) $\delta 152.3$ 150.8, 146.0, 128.2, 127.5 (q, J = 3.9 Hz), 125.0, $124.8-124.0$ (m), 118.6.

${ }^{19} \mathrm{~F}$ NMR (376 MHz, Acetone- $\left.\mathrm{d}_{6}\right) \delta-62.36(\mathrm{~s})$.

HRMS (APCl) Calculated for $\mathrm{C}_{13} \mathrm{H}_{9} \mathrm{~F}_{3} \mathrm{~N}_{2} \mathrm{O}_{3} \mathrm{~S}: 330.0280$. Found: $330.0280\{\mathrm{M}]^{+}$

MP $148-152^{\circ} \mathrm{C}$

$N$-(naphthalen-1-yl)-4-nitrobenzenesulfinamide (3m)<smiles>O=[N+]([O-])c1ccc(S(=O)Nc2cccc3ccccc23)cc1</smiles> 
Synthesised according to general procedure A (column conditions: 0-40\% EtOAc in hexane). The pure product was afforded as a brown solid (113 $\mathrm{mg}, 18 \%$ yield).

${ }^{1} \mathrm{H}$ NMR $\left(500 \mathrm{MHz}, \mathrm{DMSO}-\mathrm{d}_{6}\right) \delta 9.79(\mathrm{~s}, 1 \mathrm{H}), 8.36(\mathrm{~d}, J=8.9 \mathrm{~Hz}, 2 \mathrm{H}), 8.22-8.16(\mathrm{~m}, 1 \mathrm{H}), 8.04(\mathrm{~d}, J=8.8$ $\mathrm{Hz}, 2 \mathrm{H}), 7.92-7.87(\mathrm{~m}, 1 \mathrm{H}), 7.65(\mathrm{~d}, J=8.1 \mathrm{~Hz}, 1 \mathrm{H}), 7.59-7.50(\mathrm{~m}, 2 \mathrm{H}), 7.46(\mathrm{~d}, J=7.5 \mathrm{~Hz}, 1 \mathrm{H}), 7.38$ $(\mathrm{t}, J=7.8 \mathrm{~Hz}, 1 \mathrm{H})$.

${ }^{13} \mathrm{C}$ NMR $\left(126 \mathrm{MHz}, \mathrm{DMSO}-\mathrm{d}_{6}\right) \delta 151.5,149.0,136.6,133.8,128.1,127.5,127.3,126.4,126.0,125.7$, $124.4,124.0,122.6,117.8$.

HRMS (ESI) Calculated for $\mathrm{C}_{16} \mathrm{H}_{12} \mathrm{~N}_{2} \mathrm{O}_{3} \mathrm{SNa}$ : 335.0466. Found: $335.0461[\mathrm{M}+\mathrm{Na}]^{+}$

MP $120-122^{\circ} \mathrm{C}$

\section{4-nitro-N-(pyridin-2-yl)benzenesulfinamide (3n)}<smiles>O=[N+]([O-])c1ccc(S(=O)Nc2ccccn2)cc1</smiles>

Synthesised according to general procedure A (column conditions: 0-40\% EtOAc in hexane). The pure product was afforded as a white solid (144 $\mathrm{mg}, 55 \%$ yield).

${ }^{1} \mathrm{H}$ NMR $\left(400 \mathrm{MHz}, \mathrm{DMSO}-\mathrm{d}_{6}\right) \delta 10.23(\mathrm{~s}, 1 \mathrm{H}), 8.43(\mathrm{~d}, J=8.6 \mathrm{~Hz}, 2 \mathrm{H}), 8.29-8.22(\mathrm{~m}, 1 \mathrm{H}), 8.04(\mathrm{~d}, J=$ $9.1 \mathrm{~Hz}, 2 \mathrm{H}), 7.73-7.64(\mathrm{~m}, 1 \mathrm{H}), 7.03-6.96(\mathrm{~m}, 1 \mathrm{H}), 6.77(\mathrm{~d}, J=8.2 \mathrm{~Hz}, 1 \mathrm{H})$.

${ }^{13}$ C NMR (101 MHz, DMSO-d 6 ) $\delta$ 154.3, 152.0, 149.1, 147.7, 138.6, 127.1, 124.1, 117.7, 110.7.

HRMS (ESI) Calculated for $\mathrm{C}_{11} \mathrm{H}_{9} \mathrm{~N}_{3} \mathrm{O}_{3} \mathrm{SNa}$ : 286.0257. Found: $286.0251[\mathrm{M}+\mathrm{Na}]^{+}$

MP $132-134^{\circ} \mathrm{C}$

\section{4-nitro-N-(quinolin-8-yl)benzenesulfinamide (3o)}<smiles>O=[N+]([O-])c1ccc(S(=O)Nc2cccc3cccnc23)cc1</smiles>

Synthesised according to general procedure A (column conditions: 0-60\% EtOAc in hexane). The pure product was afforded as a brown solid (113 $\mathrm{mg}, 36 \%$ yield).

${ }^{1} \mathrm{H}$ NMR $(400 \mathrm{MHz} \text {, DMSO-d })_{6} \delta 9.99(\mathrm{~s}, 1 \mathrm{H}), 8.90(\mathrm{dd}, J=4.2,1.7 \mathrm{~Hz}, 1 \mathrm{H}), 8.43-8.31(\mathrm{~m}, 3 \mathrm{H}), 8.00(\mathrm{~d}$, $J=8.9 \mathrm{~Hz}, 2 \mathrm{H}), 7.64-7.56(\mathrm{~m}, 1 \mathrm{H}), 7.56-7.49(\mathrm{~m}, 1 \mathrm{H}), 7.44-7.34(\mathrm{~m}, 2 \mathrm{H})$.

${ }^{13} \mathrm{C}$ NMR (101 MHz, DMSO-d 6 ) $\delta 151.6,149.0,139.0,136.8,136.4,128.3,127.6,127.5,126.6,124.2$, 122.2, 121.3, 114.7.

HRMS (ESI) Calculated for $\mathrm{C}_{15} \mathrm{H}_{11} \mathrm{~N}_{3} \mathrm{O}_{3} \mathrm{SNa}$ : 336.0413. Found: $336.0410[\mathrm{M}+\mathrm{Na}]^{+}$ 


\section{N-mesityl-4-nitrobenzenesulfinamide (3p)}<smiles>Cc1cc(C)c(NS(=O)c2ccc([N+](=O)[O-])cc2)c(C)c1</smiles>

Synthesised according to general procedure A (column conditions: 0-30\% EtOAc in hexane). The pure product was afforded as an off-white solid (166 mg, 55\% yield).

${ }^{1} \mathrm{H}$ NMR $\left(500 \mathrm{MHz}, \mathrm{DMSO}-\mathrm{d}_{6}\right) \delta 8.45(\mathrm{~d}, J=8.8 \mathrm{~Hz}, 2 \mathrm{H}), 8.35(\mathrm{~s}, 1 \mathrm{H}), 8.11(\mathrm{~d}, J=9.0 \mathrm{~Hz}, 2 \mathrm{H}), 6.91(\mathrm{~s}$, $2 \mathrm{H}), 2.32(\mathrm{~s}, 6 \mathrm{H}), 2.22(\mathrm{~s}, 3 \mathrm{H})$.

${ }^{13}$ C NMR (126 MHz, DMSO-d 6 ) $\delta$ 152.6, 149.1, 135.5, 135.1, 134.5, 129.1, 127.2, 124.1, 20.4, 19.0.

HRMS (ESI) Calculated for $\mathrm{C}_{15} \mathrm{H}_{15} \mathrm{~N}_{2} \mathrm{O}_{3} \mathrm{~S}:$ 303.0798. Found: $303.0798[\mathrm{M}-\mathrm{H}]^{-}$

MP $150-152{ }^{\circ} \mathrm{C}$

N-(2,6-diisopropylphenyl)-4-nitrobenzenesulfinamide (3q)<smiles>CC(C)c1cccc(C(C)C)c1NS(=O)c1ccc([N+](=O)[O-])cc1</smiles>

Synthesised according to general procedure A (column conditions: 0-30\% EtOAc in hexane). The pure product was afforded as a yellow solid (167 $\mathrm{mg}, 24 \%$ yield).

${ }^{1}$ H NMR $(400 \mathrm{MHz}$, DMSO-d 6 ) $\delta 8.56(\mathrm{~s}, 1 \mathrm{H}), 8.48(\mathrm{~d}, J=9.3 \mathrm{~Hz}, 2 \mathrm{H}), 8.08(\mathrm{~d}, J=9.2 \mathrm{~Hz}, 2 \mathrm{H}), 7.30-7.17$ $(\mathrm{m}, 3 \mathrm{H}), 3.52(\mathrm{p}, J=6.8 \mathrm{~Hz}, 2 \mathrm{H}), 1.17(\mathrm{dd}, J=9.9,6.8 \mathrm{~Hz}, 12 \mathrm{H})$.

${ }^{13}$ C NMR (101 MHz, DMSO-d $)$ ) 152.6, 149.1, 146.7, 133.6, 127.5, 127.0, 124.2, 123.8, 27.6, 24.1, 24.0.

HRMS (ESI) Calculated for $\mathrm{C}_{18} \mathrm{H}_{21} \mathrm{~N}_{2} \mathrm{O}_{3} \mathrm{~S}: 345.1273$. Found: $345.1278[\mathrm{M}-\mathrm{H}]$

MP $90-92^{\circ} \mathrm{C}$

N-(2-(tert-butyl)phenyl)-4-nitrobenzenesulfinamide (3r)<smiles>CC(C)(C)c1ccccc1NS(=O)c1ccc([N+](=O)[O-])cc1</smiles>

Synthesised according to general procedure A (column conditions: 0-40\% EtOAc in hexane). The pure product was afforded as a beige solid ( $179 \mathrm{mg}, 56 \%$ yield). 
${ }^{1} \mathrm{H}$ NMR (400 MHz, DMSO-d 6 ) $\delta 8.51(\mathrm{~s}, 1 \mathrm{H}), 8.42(\mathrm{~d}, J=9.0 \mathrm{~Hz}, 2 \mathrm{H}), 8.03(\mathrm{~d}, J=8.6 \mathrm{~Hz}, 2 \mathrm{H}), 7.50$ (dd, $J$ $=7.5,1.9 \mathrm{~Hz}, 1 \mathrm{H}), 7.39-7.33(\mathrm{~m}, 1 \mathrm{H}), 7.21-7.08(\mathrm{~m}, 2 \mathrm{H}), 1.38(\mathrm{~s}, 9 \mathrm{H})$.

${ }^{13} \mathrm{C}$ NMR (101 MHz, DMSO-d 6 ) $\delta$ 151.8, 149.0, 143.9, 139.1, 127.31, 127.3, 126.9, 126.6, 125.8, 124.1, $34.9,30.8$.

HRMS (ESI) Calculated for $\mathrm{C}_{16} \mathrm{H}_{18} \mathrm{~N}_{2} \mathrm{O}_{3} \mathrm{SNa}$ : 341.0930 . Found: 341.0917 [M+Na] ${ }^{+}$

MP $96-98^{\circ} \mathrm{C}$

\section{2-methoxy-4-nitro-N-phenylbenzenesulfinamide (3s)}<smiles>COc1cc([N+](=O)[O-])ccc1S(=O)Nc1ccccc1</smiles>

Synthesised by adaptation of a literature procedure. ${ }^{11} \mathrm{~A} 100 \mathrm{~mL}$ round-bottomed flask was charged with 2-methoxy-4-nitrobenzenesulfonyl chloride ( $1 \mathrm{~g}, 4 \mathrm{mmol})$, evacuated under vacuum and filled with nitrogen. Anhydrous DCM ( $10 \mathrm{~mL})$ was added, alongside $\mathrm{NEt}_{3}\left(8 \mathrm{mmol}_{3} 1.12 \mathrm{~mL}\right)$ and the solution was stirred at $0^{\circ} \mathrm{C}$. A separate $100 \mathrm{~mL}$ round-bottomed flask was charged with $\mathrm{PPh}_{3}(1.052 \mathrm{~g}, 4 \mathrm{mmol})$, evacuated under vacuum and filled with nitrogen. Anhydrous DCM $(10 \mathrm{~mL})$ and aniline $(365 \mu \mathrm{L}, 4$ $\mathrm{mmol}$ ) was added and the solution was stirred at $0^{\circ} \mathrm{C}$. The contents of the second flask were added dropwise to the first flask over ice, and the reaction mixture was left stirring for 16 hours. The reaction mixture was extracted with DCM $(20 \mathrm{~mL})$, washed with $\mathrm{H}_{2} \mathrm{O}(3 \times 20 \mathrm{~mL})$, dried with anhydrous $\mathrm{MgSO}_{4}$. The organic layers were then filtered, concentrated in vacuo and purified by flash column chromatography (0-40\% EtOAc in hexane). The pure product was afforded as a yellow solid ( $247 \mathrm{mg}$, $21 \%$ yield).

${ }^{1} \mathrm{H}$ NMR (500 MHz, DMSO-d 6 ) $\delta 9.28(\mathrm{~s}, 1 \mathrm{H}), 8.07-7.97(\mathrm{~m}, 2 \mathrm{H}), 7.89(\mathrm{~d}, J=1.9 \mathrm{~Hz}, 1 \mathrm{H}), 7.24(\mathrm{t}, 2 \mathrm{H})$, $7.02(\mathrm{~d}, J=7.7 \mathrm{~Hz}, 2 \mathrm{H}), 6.95(\mathrm{t}, J=7.4 \mathrm{~Hz}, 1 \mathrm{H}), 3.94(\mathrm{~s}, 3 \mathrm{H})$.

${ }^{13}$ C NMR $(126 \mathrm{MHz}$, DMSO-d 6 ) $\delta 156.3,150.6,142.0,138.6,129.2,127.1,122.3,117.7,115.5,106.9$, 57.0 .

HRMS (ESI) Calculated for $\mathrm{C}_{13} \mathrm{H}_{12} \mathrm{~N}_{2} \mathrm{O}_{4} \mathrm{SNa}$ : 315.0410 . Found: $315.0405[\mathrm{M}+\mathrm{Na}]^{+}$

MP $104-106^{\circ} \mathrm{C}$

\section{5-nitro-N-phenylpyridine-2-sulfinamide (3t)}<smiles>O=[N+]([O-])c1ccc(S(=O)Nc2ccccc2)nc1</smiles>

Synthesised by adaptation of a literature procedure. ${ }^{10} \mathrm{~A} 50 \mathrm{~mL}$ round-bottomed flask was charged with $\mathrm{KF}(1 \mathrm{mmol}, 59 \mathrm{mg})$. $\mathrm{CH}_{3} \mathrm{CN}(4.2 \mathrm{~mL})$ and $\mathrm{H}_{2} \mathrm{O}(0.8 \mathrm{~mL})$ were added and the flask was stirred at 0 ${ }^{\circ} \mathrm{C}$. Meta-chloroperoxybenzoic acid, 70\% (m-CBPA) $(1 \mathrm{mmol}, 172 \mathrm{mg}$ ) was added slowly and the mixture was left to stir for $30 \mathrm{~min}$. Sulfenamide $3 \mathrm{tp}(0.5 \mathrm{mmol}, 132 \mathrm{mg})$ was added portion-wise and 
the reaction mixture was stirred at $0{ }^{\circ} \mathrm{C}$ for $30 \mathrm{~min}$. The reaction mixture was then extracted with EtOAc $(20 \mathrm{~mL})$, washed with saturated aqueous $\mathrm{NaHCO}_{3}(3 \times 20 \mathrm{~mL})$ and dried with anhydrous $\mathrm{MgSO}_{4}$. The organic layers were then filtered, concentrated in vacuo and purified by flash column chromatography (0-50\% EtOAc in hexane). The pure product was afforded as a white solid (58 $\mathrm{mg}$, $45 \%$ yield).

${ }^{1} \mathrm{H}$ NMR $\left(500 \mathrm{MHz}, \mathrm{DMSO}-\mathrm{d}_{6}\right) \delta 9.71(\mathrm{~s}, 1 \mathrm{H}), 9.48-9.40(\mathrm{~m}, 1 \mathrm{H}), 8.84(\mathrm{dd}, J=8.7,2.6 \mathrm{~Hz}, 1 \mathrm{H}), 8.26$ (dd, $J=8.5,0.7 \mathrm{~Hz}, 1 \mathrm{H}), 7.22(\mathrm{t}, J=7.9 \mathrm{~Hz}, 2 \mathrm{H}), 7.04(\mathrm{~d}, J=7.4 \mathrm{~Hz}, 2 \mathrm{H}), 6.93(\mathrm{t}, J=7.4 \mathrm{~Hz}, 1 \mathrm{H})$.

${ }^{13}$ C NMR (126 MHz, DMSO-d 6 ) $\delta$ 168.8, 145.1, 144.9, 141.2, 133.5, 129.3, 122.5, 121.9, 117.7.

HRMS (APCI) Calculated for $\mathrm{C}_{11} \mathrm{H}_{10} \mathrm{~N}_{3} \mathrm{O}_{3} \mathrm{~S}: 264.0437$. Found: $264.0430[\mathrm{M}+\mathrm{H}]^{+}$

MP $155-158^{\circ} \mathrm{C}$

\section{Compounds Synthesised for Mechanistic investigations}

4-nitro-N-phenylbenzenesulfonamide (1a)<smiles>O=[N+]([O-])c1ccc(NS(=O)(=O)c2ccccc2)cc1</smiles>

Prepared according to a literature procedure. ${ }^{12}$ A $50 \mathrm{~mL}$ round-bottomed flask was charged with 4nitrobenzenesulfonyl chloride ( $244 \mathrm{mg}, 1.1 \mathrm{mmol}$ ), which was evacuated under vacuum and filled with nitrogen. Pyridine $(5 \mathrm{~mL})$ was added, alongside aniline $(91 \mu \mathrm{L}, 1 \mathrm{mmol})$, and the reaction mixture was stirred at room temperature for 3 hours. Toluene $(10 \mathrm{~mL})$ was added to the reaction mixture and the solvent was evaporated in vacuo. The reaction mixture was then dissolved in DCM ( $20 \mathrm{~mL})$, extracted from $\mathrm{H}_{2} \mathrm{O}(2 \times 20 \mathrm{~mL})$, dried with anhydrous $\mathrm{MgSO}_{4}$, filtered and concentrated in vacuo. The crude product was then purified by flash column chromatography (10-30\% EtOAc in hexane) affording the pure product as a white solid (126 mg, 45\%).

${ }^{1} \mathrm{H}$ NMR $\left(500 \mathrm{MHz}\right.$, Acetone- $\left.\mathrm{d}_{6}\right) \delta 9.29(\mathrm{~s}, 1 \mathrm{H}), 8.38(\mathrm{~d}, J=8.6 \mathrm{~Hz}, 2 \mathrm{H}), 8.05(\mathrm{~d}, J=9.0 \mathrm{~Hz}, 2 \mathrm{H}), 7.29(\mathrm{t}, J$ $=7.2 \mathrm{~Hz}, 2 \mathrm{H}), 7.23(\mathrm{~d}, J=7.6 \mathrm{~Hz}, 2 \mathrm{H}), 7.14(\mathrm{t}, J=6.9 \mathrm{~Hz}, 1 \mathrm{H})$.

${ }^{13} \mathrm{C}$ NMR $\left(126 \mathrm{MHz}\right.$, Acetone- $\left.\mathrm{d}_{6}\right) \delta 151.2,146.2,137.9,130.2,129.5,126.2,125.2,122.4$.

HRMS (APCI) Calculated for $\mathrm{C}_{12} \mathrm{H}_{9} \mathrm{~N}_{2} \mathrm{O}_{4} \mathrm{~S}: 277.0289$. Found: 277.0280 [M-H]

Data is in accordance with literature. ${ }^{12}$

\section{N-methyl-4-nitro-N-phenylbenzenesulfinamide (3u)}<smiles>CN(c1ccccc1)S(=O)c1ccc([N+](=O)[O-])cc1</smiles> 
Synthesised according to general procedure A (column conditions: 5-40\% EtOAc in hexane). The pure product was afforded as a brown solid (138 $\mathrm{mg}, 50 \%$ yield).

${ }^{1} \mathrm{H}$ NMR $\left(500 \mathrm{MHz}\right.$, Acetone- $\left.\mathrm{d}_{6}\right) \delta 8.46(\mathrm{~d}, J=9.0 \mathrm{~Hz}, 2 \mathrm{H}), 8.03(\mathrm{~d}, J=8.2 \mathrm{~Hz}, 2 \mathrm{H}), 7.45-7.36(\mathrm{~m}, 4 \mathrm{H})$, $7.23-7.16(\mathrm{~m}, 1 \mathrm{H}), 2.90(\mathrm{~s}, 3 \mathrm{H})$.

${ }^{13}$ C NMR (126 MHz, Acetone- $\left.d_{6}\right) \delta$ 151.6, 146.6, 130.3, 128.3, 125.4, 125.1, 122.1, 31.6.

HRMS (APCI) Calculated for $\mathrm{C}_{13} \mathrm{H}_{13} \mathrm{~N}_{2} \mathrm{O}_{3} \mathrm{~S}: 277.0641$. Found: $277.0640[\mathrm{M}+\mathrm{H}]^{+}$

MP $90-95^{\circ} \mathrm{C}$

\section{2-((4-nitrophenyl)thio)aniline (11)}<smiles>Nc1ccccc1Sc1ccc([N+](=O)[O-])cc1</smiles>

A $100 \mathrm{~mL}$ round-bottomed flask was charged with 4-fluoronitrobenzene $\left(282 \mathrm{mg}, 2 \mathrm{mmol}\right.$ ) and $\mathrm{K}_{2} \mathrm{CO}_{3}$ ( $553 \mathrm{mg}, 4 \mathrm{mmol}$ ), which was evacuated under vacuum and filled with nitrogen. Anhydrous DMF (10 $\mathrm{mL}$ ) was added to the mixture which was stirred in a pre-warmed oil bath at $70{ }^{\circ} \mathrm{C}$. 2aminobenzenethiol $(0.207 \mathrm{~mL}, 2 \mathrm{mmol})$ was added dropwise and the mixture was left to stir at $70^{\circ} \mathrm{C}$ for $1 \mathrm{~h}$. The reaction mixture was dissolved in EtOAc $(20 \mathrm{~mL})$ and washed with $10 \%(\mathrm{w} / \mathrm{v})$ aqueous $\mathrm{LiCl}$ $(3 \times 20 \mathrm{~mL})$. The aqueous layer was then extracted with EtOAc $(3 \times 20 \mathrm{~mL})$ and the combined organic layers were dried with anhydrous $\mathrm{MgSO}_{4}$, filtered and concentrated in vacuo. The crude product was then purified by flash column chromatography (0-40\% EtOAc in hexane), affording the pure product as a brown solid (456 $\mathrm{mg}, 93 \%$ yield).

${ }^{1} \mathrm{H}$ NMR $\left(500 \mathrm{MHz}, \mathrm{CDCl}_{3}\right) \delta 8.06(\mathrm{~d}, J=9.2 \mathrm{~Hz}, 2 \mathrm{H}), 7.47-7.42(\mathrm{~m}, 1 \mathrm{H}), 7.36-7.29(\mathrm{~m}, 1 \mathrm{H}), 7.11(\mathrm{~d}, J$ $=9.1 \mathrm{~Hz}, 2 \mathrm{H}), 6.86-6.83(\mathrm{~m}, 1 \mathrm{H}), 6.83-6.78(\mathrm{~m}, 1 \mathrm{H}), 4.29(\mathrm{~s}, 2 \mathrm{H})$.

${ }^{13} \mathrm{C}$ NMR $\left(126 \mathrm{MHz}, \mathrm{CDCl}_{3}\right) \delta 149.2,147.3,145.4,137.8,132.5,125.6,124.2,119.3,115.8,111.4$.

HRMS (ESI) Calculated for $\mathrm{C}_{12} \mathrm{H}_{10} \mathrm{~N}_{2} \mathrm{O}_{2} \mathrm{SNa}$ : 269.0355. Found: 269.0347 [M+Na] ${ }^{+}$

Data is in accordance with literature. ${ }^{13}$

\section{2-((4-nitrophenyl)sulfinyl)aniline (10)}<smiles>Nc1ccccc1S(=O)c1ccc([N+](=O)[O-])cc1</smiles>

A $100 \mathrm{~mL}$ round-bottomed flask was charged with 2-((4-nitrophenyl)thio)aniline 11 (440 mg, 1.78 $\mathrm{mmol})$, evacuated under vacuum and filled with nitrogen. $D C M(10 \mathrm{~mL})$ was added and the mixture was stirred at $0{ }^{\circ} \mathrm{C}$. A second $100 \mathrm{~mL}$ round-bottomed flask was charged with Metachloroperoxybenzoic acid, $70 \%$ ( $m$-CBPA) $(230 \mathrm{mg}, 1.9 \mathrm{mmol})$, evacuated under vacuum and filled with nitrogen. DCM $(10 \mathrm{~mL})$ was added and the contents of this flask were added slowly to the first flask. The reaction mixture was stirred at $0{ }^{\circ} \mathrm{C}$ for $1 \mathrm{~h}$, at which point the ice bath was removed and the mixture was allowed to warm to room temperature. On consumption of starting material (monitored 
by $T L C)$ the reaction was quenched with saturated aqueous $\mathrm{NaHCO}_{3}(20 \mathrm{~mL})$ and extracted with $\mathrm{DCM}$ $(2 \times 20 \mathrm{~mL})$. The combined organic layers were then dried with anhydrous $\mathrm{MgSO}_{4}$, filtered and concentrated in vacuo. The crude product was then purified by flash column chromatography (20-60\% EtOAc in hexane), affording the pure product as a yellow solid (163 mg, 35\% yield).

${ }^{1} \mathrm{H}$ NMR $\left(500 \mathrm{MHz}, \mathrm{CDCl}_{3}\right) \delta 8.29(\mathrm{~d}, J=9.1 \mathrm{~Hz}, 2 \mathrm{H}), 7.72(\mathrm{~d}, J=8.8 \mathrm{~Hz}, 2 \mathrm{H}), 7.49$ (dd, $J=7.7,1.5 \mathrm{~Hz}$, $1 \mathrm{H}), 7.32-7.25(\mathrm{~m}, 1 \mathrm{H}), 6.82(\mathrm{td}, J=7.6,1.1 \mathrm{~Hz}, 1 \mathrm{H}), 6.64-6.58(\mathrm{~m}, 1 \mathrm{H}), 4.89(\mathrm{~s}, 2 \mathrm{H})$.

${ }^{13} \mathrm{C}$ NMR $\left(126 \mathrm{MHz}, \mathrm{CDCl}_{3}\right) \delta 150.9,149.1,148.0,134.0,128.6,125.9,124.0,122.6,118.0,117.8$.

HRMS (ESI) Calculated for $\mathrm{C}_{12} \mathrm{H}_{10} \mathrm{~N}_{2} \mathrm{O}_{3} \mathrm{SNa}$ : 285.0304. Found: $285.0296[\mathrm{M}+\mathrm{Na}]^{+}$

\section{Sulfinamide Starting Material Precursors}

\section{Methyl 4-nitrobenzenesulfinate (4)}<smiles>COS(=O)c1ccc([N+](=O)[O-])cc1</smiles>

Prepared according to a literature procedure ${ }^{14} \mathrm{~A} 250 \mathrm{ml}$ round-bottomed flask was charged with 1,2bis(4-nitrophenyl)disulfane $(2.48 \mathrm{~g}, 8 \mathrm{mmol})$, which was then dissolved in $\mathrm{MeOH}(50 \mathrm{~mL})$ and DCM (50 $\mathrm{mL}$ ). The solution was stirred at $0{ }^{\circ} \mathrm{C}$, and then $\mathrm{N}$-bromosuccinimide $(7.2 \mathrm{~g}, 40 \mathrm{mmol})$ was added slowly. The reaction mixture was stirred overnight. The reaction mixture was then extracted with DCM (50 $\mathrm{mL}$ ), washed with saturated aqueous $\mathrm{NaHCO}_{3}(2 \times 50 \mathrm{~mL})$, dried with anhydrous $\mathrm{Na}_{2} \mathrm{SO}_{4}$, filtered and concentrated in vacuo. The crude product was purified by flash column chromatography (0-30\% EtOAc in hexane) to afford a light yellow solid ( $2.752 \mathrm{~g}, 85 \%$ yield).

${ }^{1} \mathrm{H}$ NMR $\left(400 \mathrm{MHz}, \mathrm{CDCl}_{3}\right) \delta 8.40(\mathrm{~d}, J=8.7 \mathrm{~Hz}), 7.90(\mathrm{~d}, J=8.6 \mathrm{~Hz}, 2 \mathrm{H}, 3.54(\mathrm{~s}, 3 \mathrm{H})$.

${ }^{13} \mathrm{C}$ NMR $\left(101 \mathrm{MHz}, \mathrm{CDCl}_{3}\right) \delta 150.3,150.2,126.9,124.3,50.7$.

HRMS (ESI) Calculated for $\mathrm{C}_{7} \mathrm{H}_{7} \mathrm{NO}_{4} \mathrm{~S}: 201.0096$ Found: 201.0101 [M] ${ }^{-}$

Data is in accordance with literature. ${ }^{15}$

\section{S-(5-nitropyridin-2-yl)-N-phenylthiohydroxylamine (7b)}<smiles>O=[N+]([O-])c1ccc(SNc2ccccc2)nc1</smiles>

Prepared by adaptation of a literature procedure. ${ }^{16}$ a $100 \mathrm{~mL}$ round-bottomed flask was charged with $\mathrm{AgNO}_{3}(350 \mathrm{mg}, 2 \mathrm{mmol}$ ) and 5-methyl-2-((5-nitropyridin-2-yl)disulfaneyl)pyridine (612 mg, $2 \mathrm{mmol})$ and the flask was evacuated under vacuum and filled with nitrogen. Anhydrous DCM $(10 \mathrm{~mL})$ and $\mathrm{MeOH}(20 \mathrm{~mL})$ were added and the flask was stirred at $0{ }^{\circ} \mathrm{C}$. Aniline $(0.9 \mathrm{~mL}, 10 \mathrm{mmol})$ was added dropwise and the reaction mixture was stirred overnight. The reaction mixture was then filtered and concentrated in vacuo. The concentrated product was then extracted with DCM $(30 \mathrm{~mL})$, washed with 
$\mathrm{H}_{2} \mathrm{O}(2 \times 20 \mathrm{~mL})$, dried with anhydrous $\mathrm{MgSO}_{4}$, filtered and concentrated in vacuo. The crude product was purified by flash column chromatography (0-30\% EtOAc in hexane) to afford a yellow solid (189 $\mathrm{mg}, 38 \%$ yield).

${ }^{1} \mathrm{H}$ NMR (500 MHz, DMSO-d 6 ) $\delta 9.23(\mathrm{~d}, J=2.4 \mathrm{~Hz}, 1 \mathrm{H}), 8.49$ (dd, $\left.J=8.9,2.6 \mathrm{~Hz}, 1 \mathrm{H}\right), 8.30(\mathrm{~s}, 1 \mathrm{H}), 7.35$ (d, $J=8.4 \mathrm{~Hz}, 1 \mathrm{H}), 7.23(\mathrm{t}, J=8.3 \mathrm{~Hz}, 2 \mathrm{H}), 7.01(\mathrm{~d}, J=7.6 \mathrm{~Hz}, 2 \mathrm{H}), 6.84(\mathrm{t}, J=7.3 \mathrm{~Hz}, 1 \mathrm{H}$ ).

${ }^{13}$ C NMR (126 MHz, DMSO-d $\left.\mathrm{d}_{6}\right) \delta$ 173.1, 146.0, 145.1, 141.5, 132.3, 129.4, 120.3, 117.0, 114.4.

HRMS (APCI) Calculated for $\mathrm{C}_{11} \mathrm{H}_{10} \mathrm{~N}_{3} \mathrm{O}_{2} \mathrm{~S}: 248.0488$. Found: $248.0477[\mathrm{M}+\mathrm{H}]^{+}$

MP $112-114^{\circ} \mathrm{C}$ 


\section{Poor-yielding and Unsuccessful Substrates}<smiles>O=[N+]([O-])c1ccc(Nc2ccc([N+](=O)[O-])c(Nc3ccc([N+](=O)[O-])c(Nc4ncccn4)c3)c2)cc1</smiles>

\section{S-Aryl Scope}<smiles>O=[N+]([O-])c1ccccc1Nc1ccccc1</smiles><smiles>c1ccc(Nc2ccccn2)cc1</smiles><smiles>O=[SH]c1nc2ccccc2s1</smiles><smiles>O=[N+]([O-])c1ccc(Nc2ccccc2)cc1</smiles><smiles>N#Cc1ccc(Nc2ccccc2)cc1</smiles><smiles>Clc1ccc(Nc2ccccc2)cc1</smiles><smiles>O=[N+]([O-])c1c(F)c(F)c(F)c(Nc2ccccc2)c1F</smiles><smiles>O=[N+]([O-])c1cc(C(F)(F)F)ccc1Nc1ccccc1</smiles> 


\section{NMR Spectra for Synthesised Compounds}

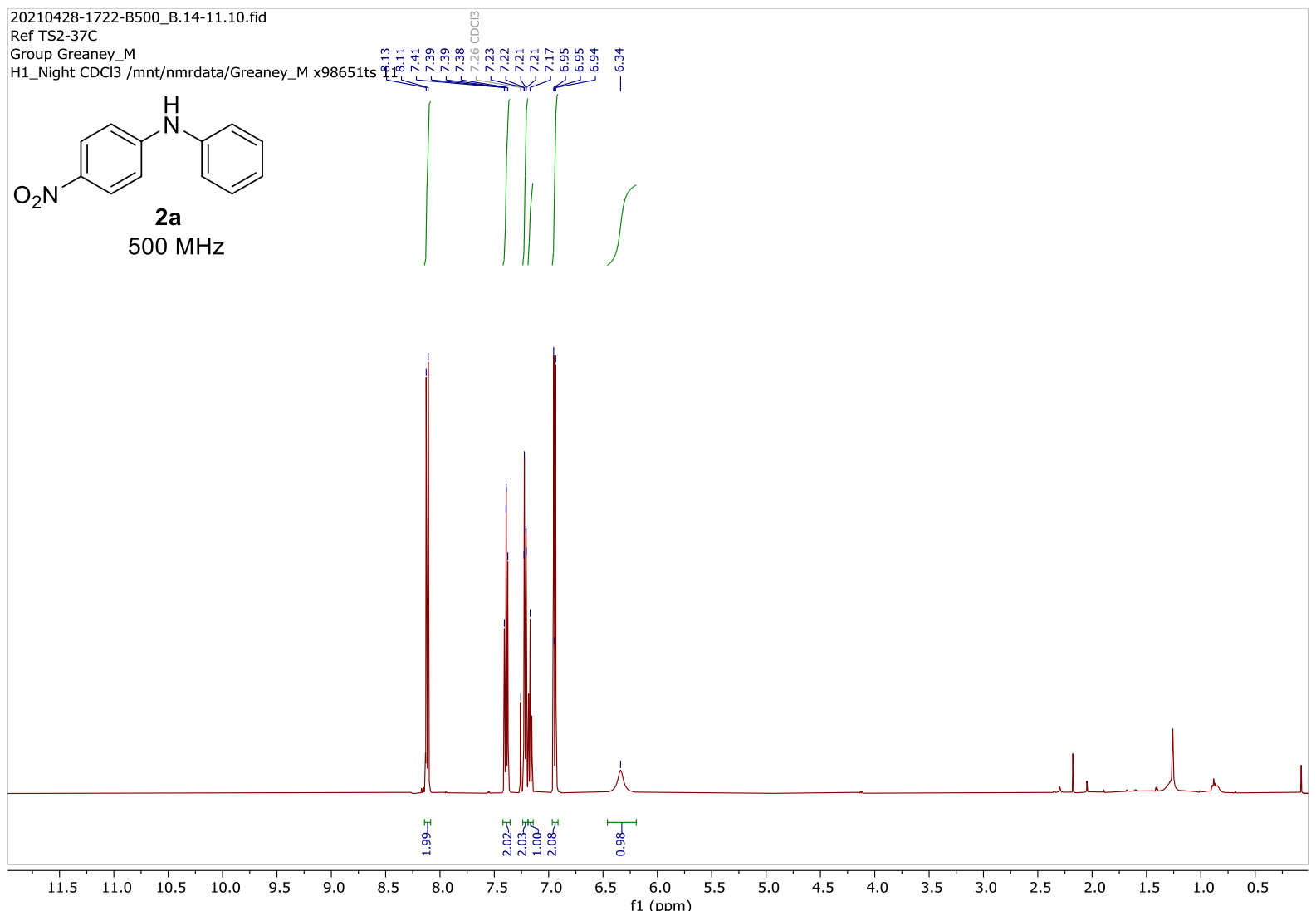

20210428-1722-B500_B.14-11.11.fid

Ref TS2-37C

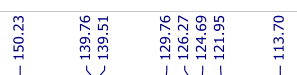

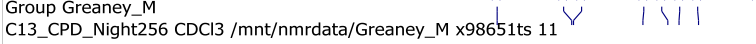<smiles>O=[N+]([O-])c1ccc(Nc2ccccc2)cc1</smiles>

$2 a$

$126 \mathrm{MHz}$

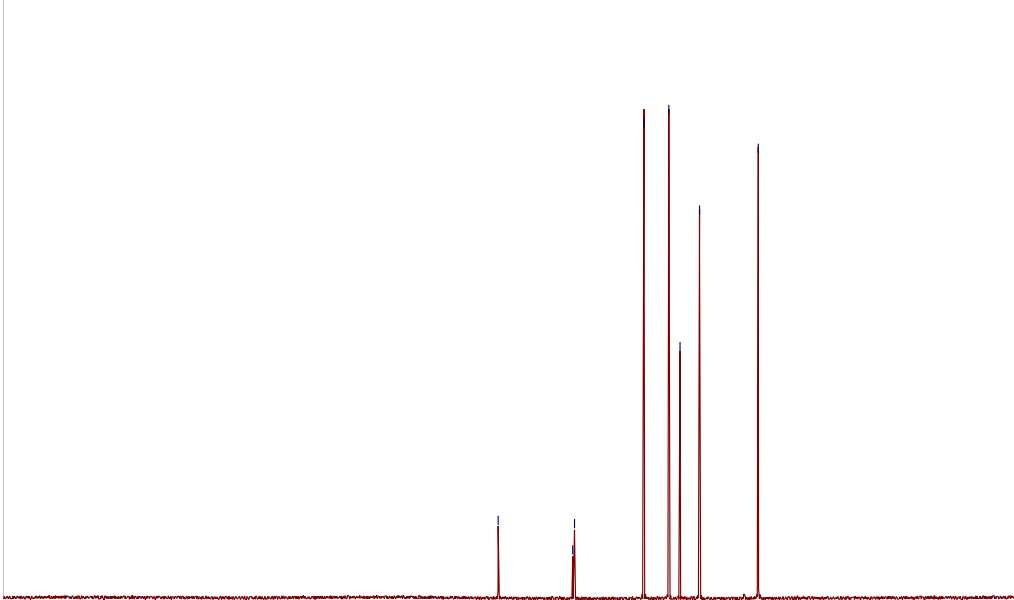

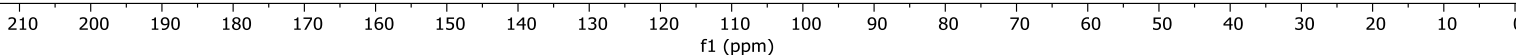


20210604-1124-B400_B.11-31.10.fid

Ref TS2-73C

Group Greaney_M

H1_Night Acetone /mnt/nmrdata/Greaney_M x98651ts 31<smiles>Cc1ccccc1Nc1ccc([N+](=O)[O-])cc1</smiles>

2b

$400 \mathrm{MHz}$
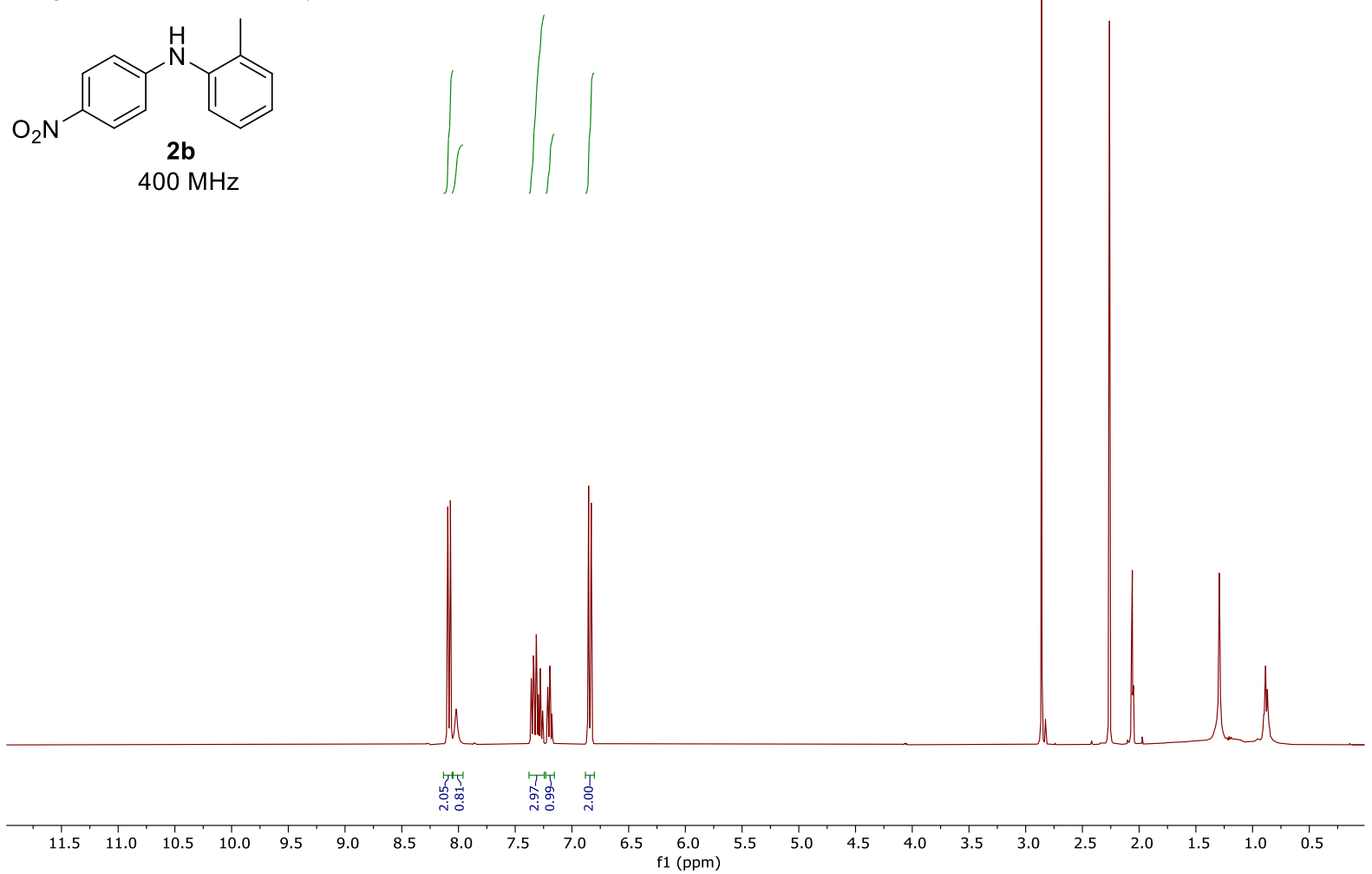

20210604-|124-B400_B.11-31.11.fid

Ref TS2-73C

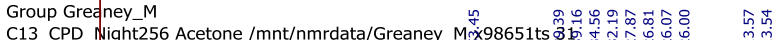

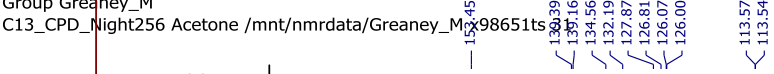

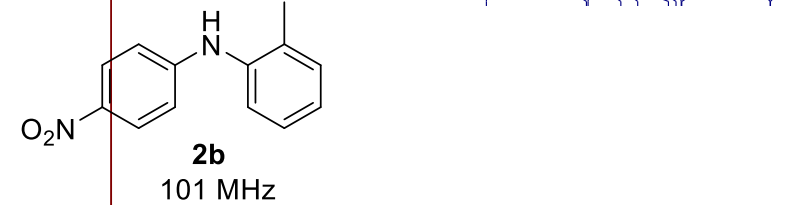

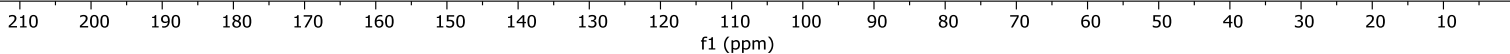


20210526-1548-B400_B.11-38.10.fid

Ref TS2-74C

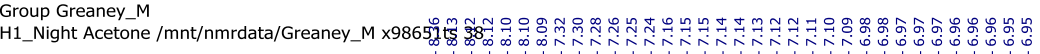<smiles>Cc1cccc(Nc2ccc([N+](=O)[O-])cc2)c1</smiles>

$400 \mathrm{MHz}$
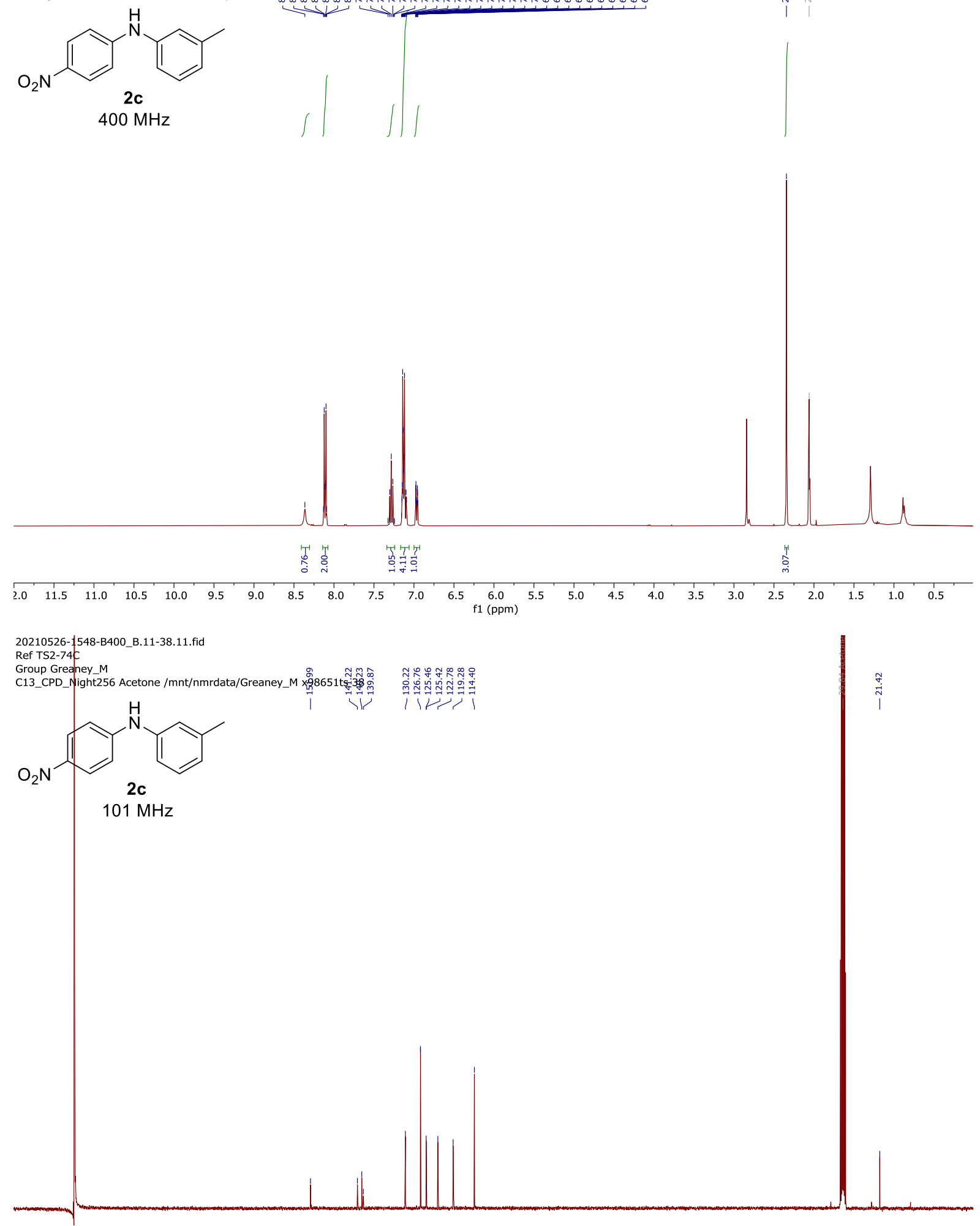

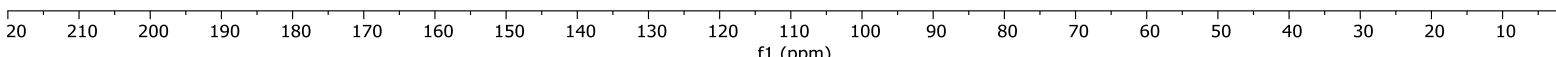


20210514-1121-B400_B.12-10.10.fid

Ref TS2-59

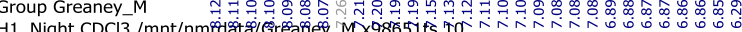

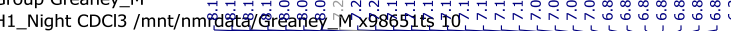<smiles>Cc1ccc(Nc2ccc([N+](=O)[O-])cc2)cc1</smiles>

2d

$400 \mathrm{MHz}$
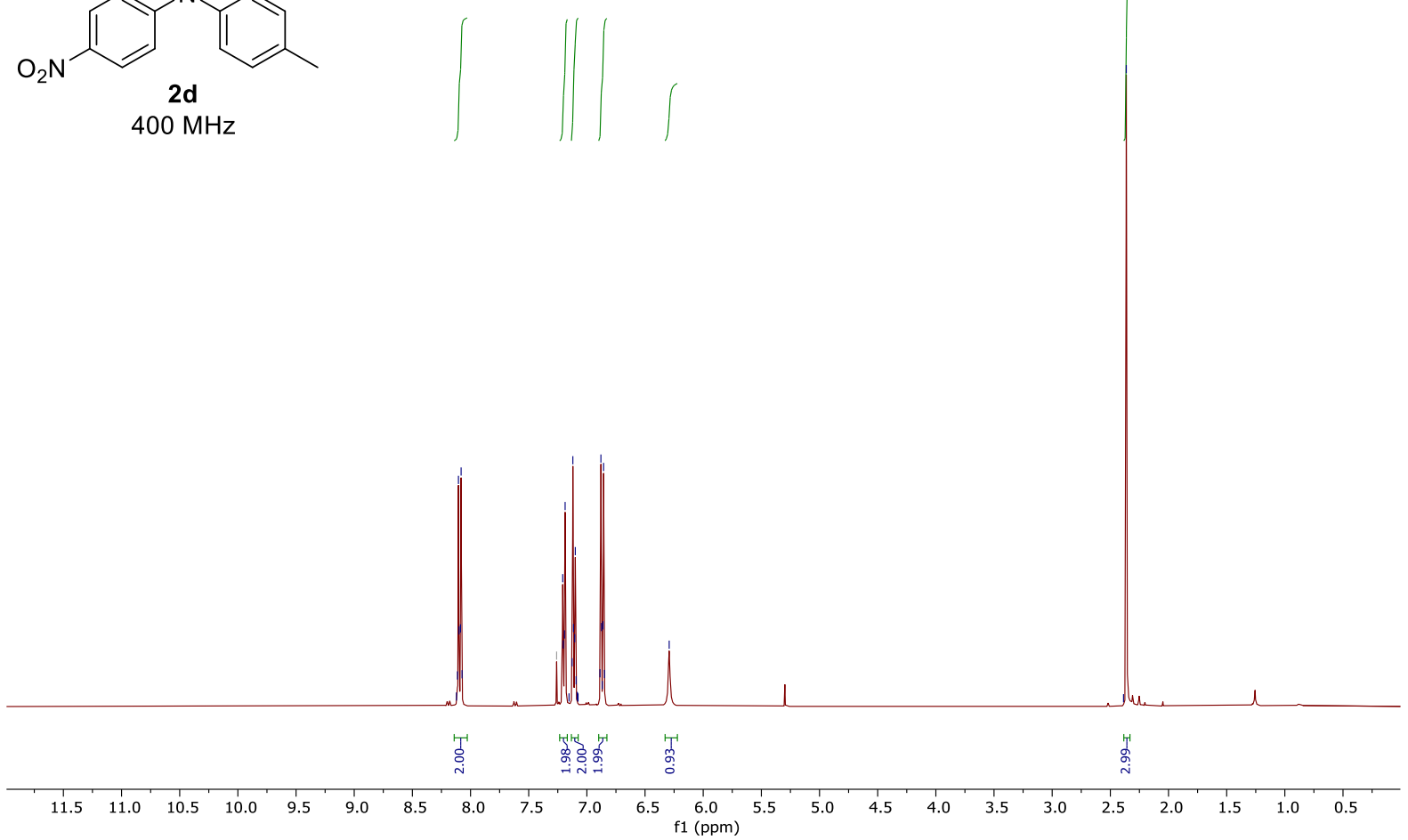

0210514-1121-B400 B.12-10.11 fid

Ref TS2-59

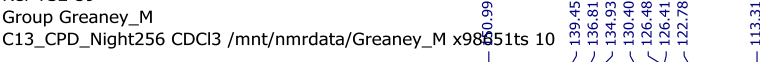<smiles>Cc1ccc(Nc2ccc([N+](=O)[O-])cc2)cc1</smiles>

$101 \mathrm{MHz}$

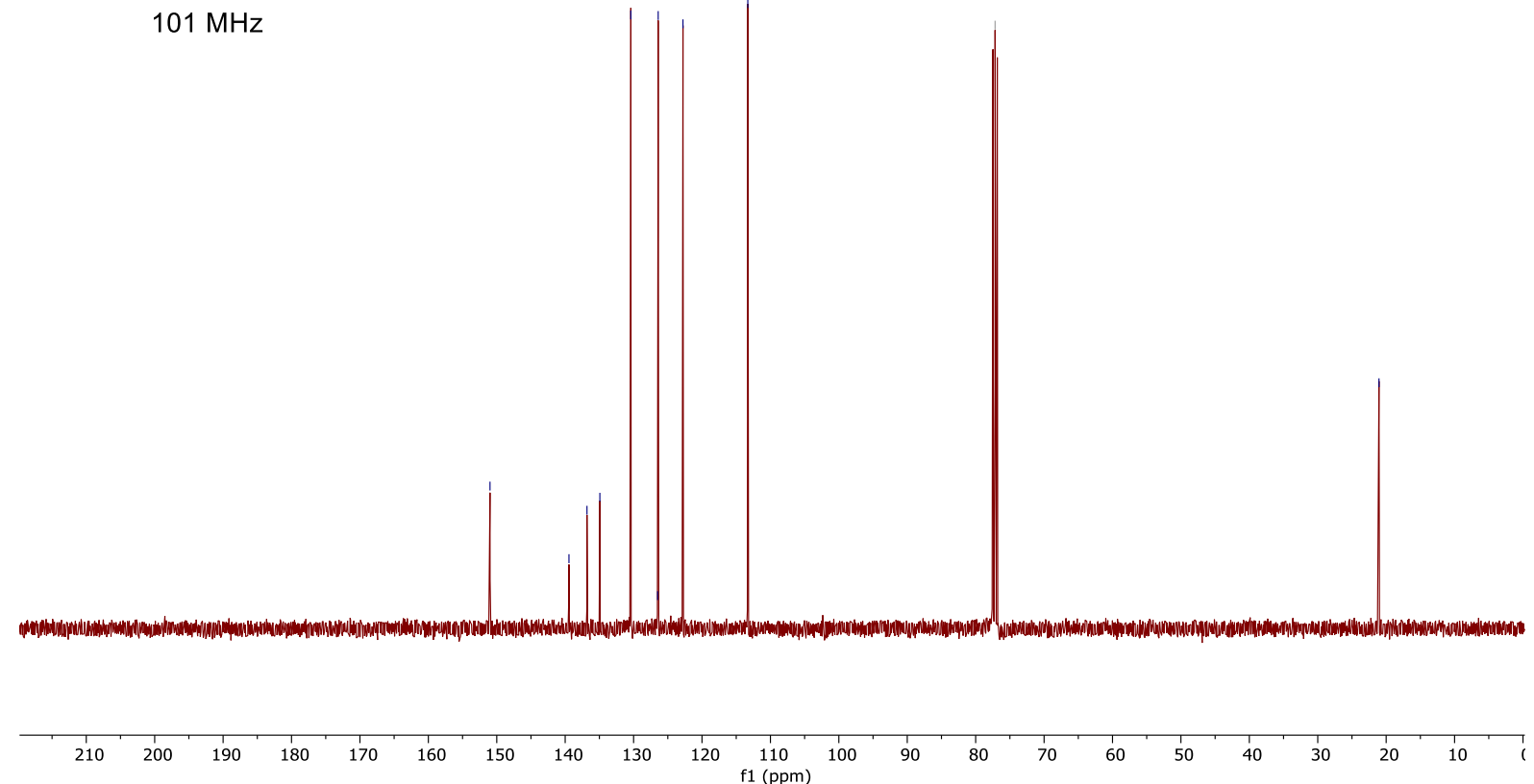


20211018-1119-B500_B.14-46.10.fid

Ref TS3-28a

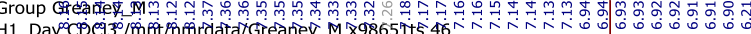

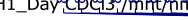<smiles>O=[N+]([O-])c1ccc(Nc2ccc(Cl)cc2)cc1</smiles>

$500 \mathrm{MHz}$

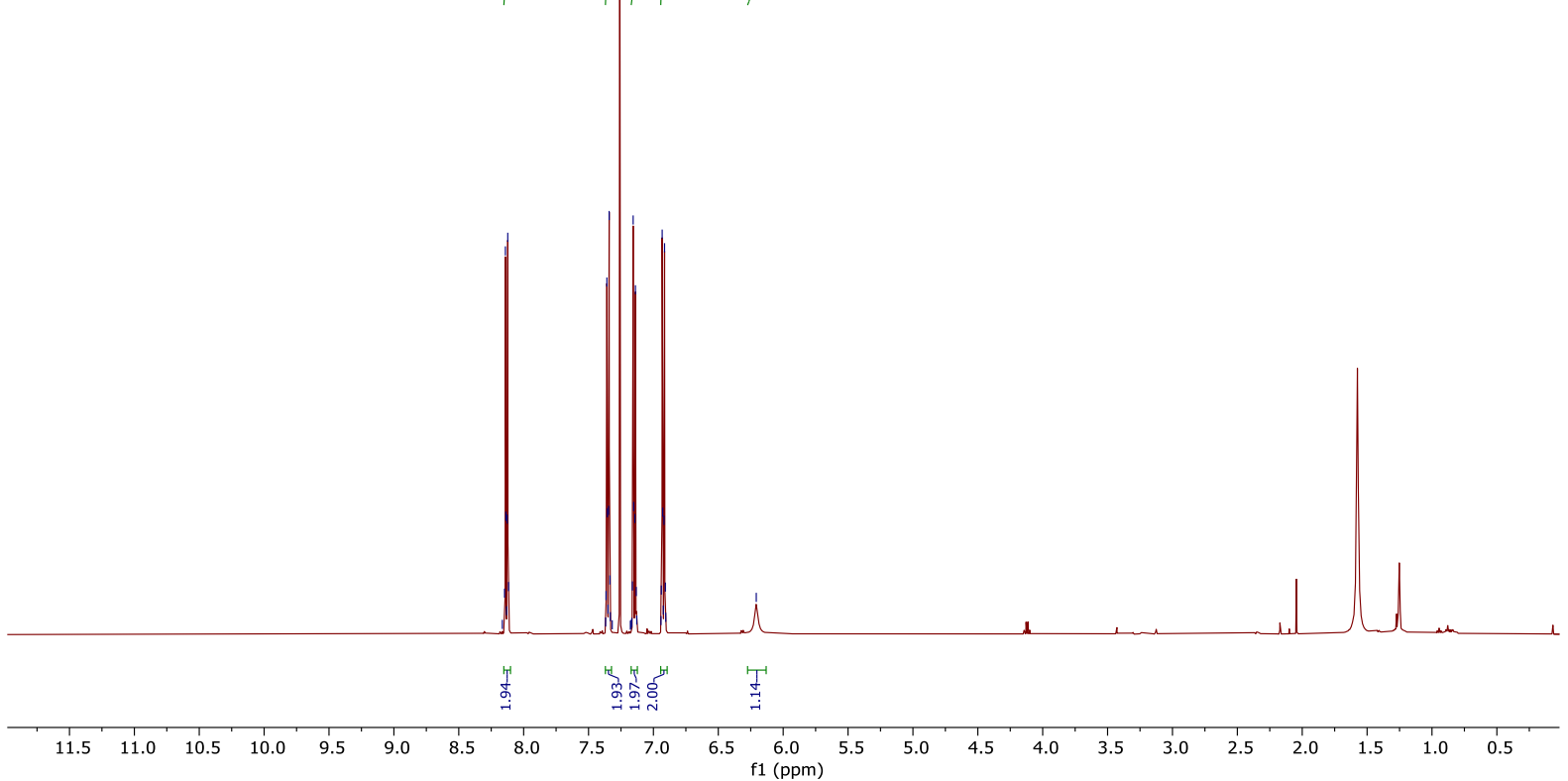

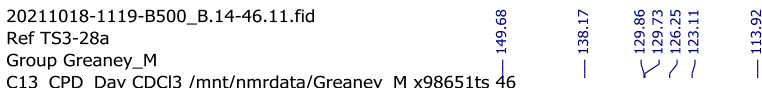<smiles>O=[N+]([O-])c1ccc(Nc2ccc(Cl)cc2)cc1</smiles>

$2 e$

$126 \mathrm{MHz}$

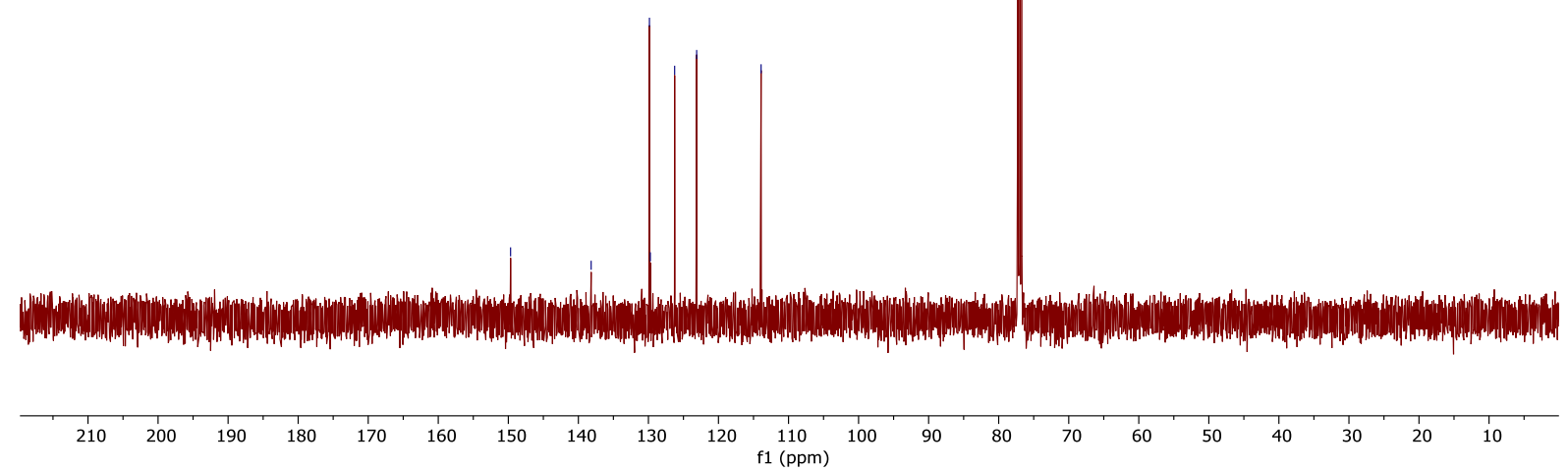


20210520-1810-B400_B.11-12.9.fid

Ref TS2-64C

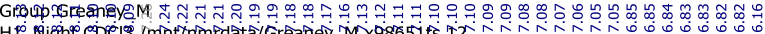

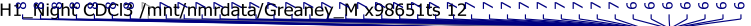

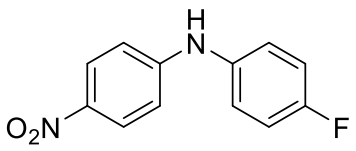

$2 f$

$400 \mathrm{MHz}$
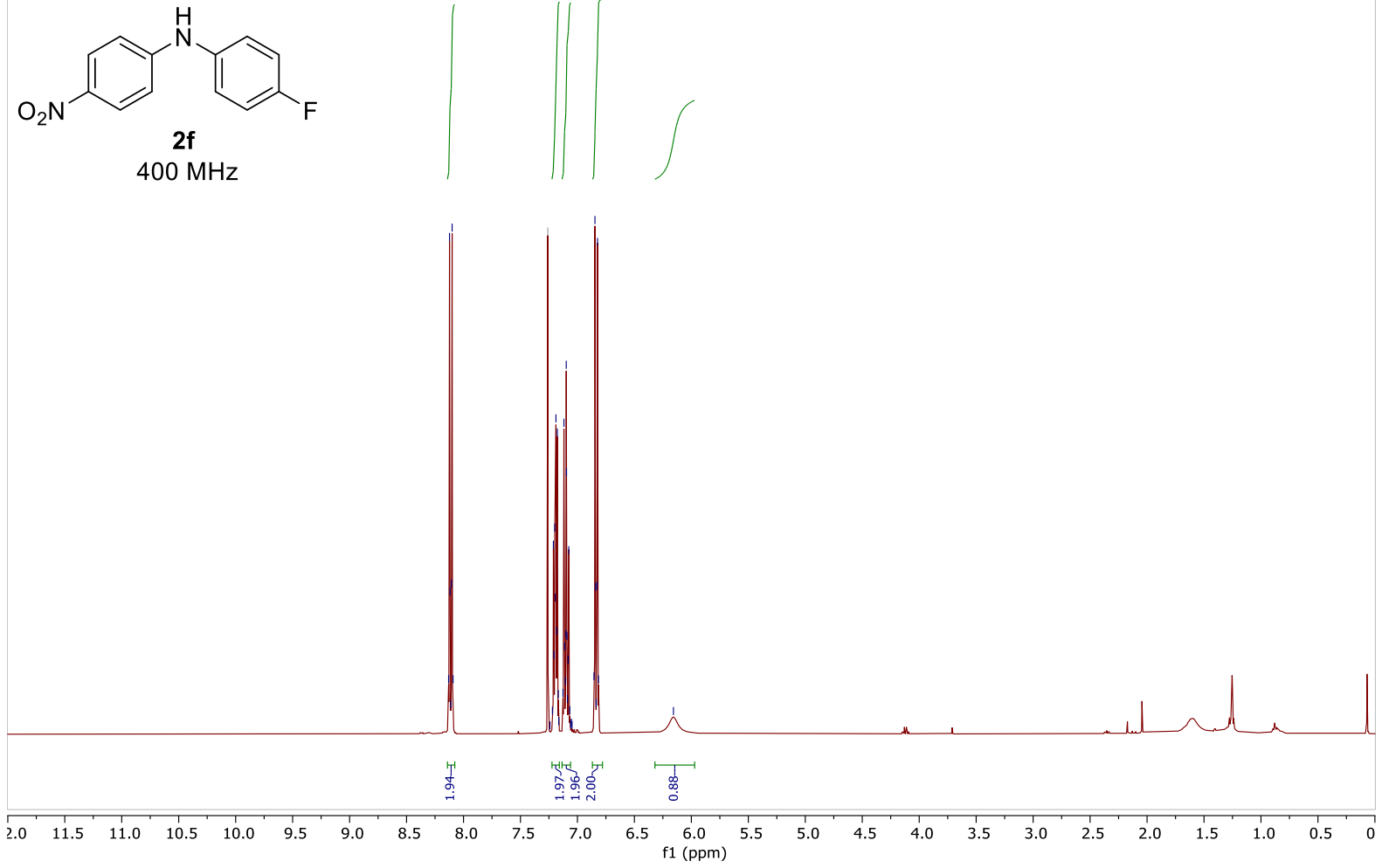

20210520-1810-B400_B.11-12.10.fid

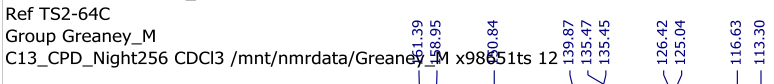<smiles></smiles>

$101 \mathrm{MHz}$

$\begin{array}{lllllllllll}210 & 200 & 190 & 180 & 170 & 160 & 150 & 140 & 130 & 120 & 110 \\ \mathrm{f} 1(\mathrm{ppm}) & 100\end{array}$ 
20210520-1810-B400_B.11-12.15.fid

Ref TS2-64C

Group Greaney_M

F19_NoCPD_Night CDCl3 /mnt/nmrdata/Greaney_M x98651ts 12<smiles>O=[N+]([O-])c1ccc(Nc2ccc(F)cc2)cc1</smiles>



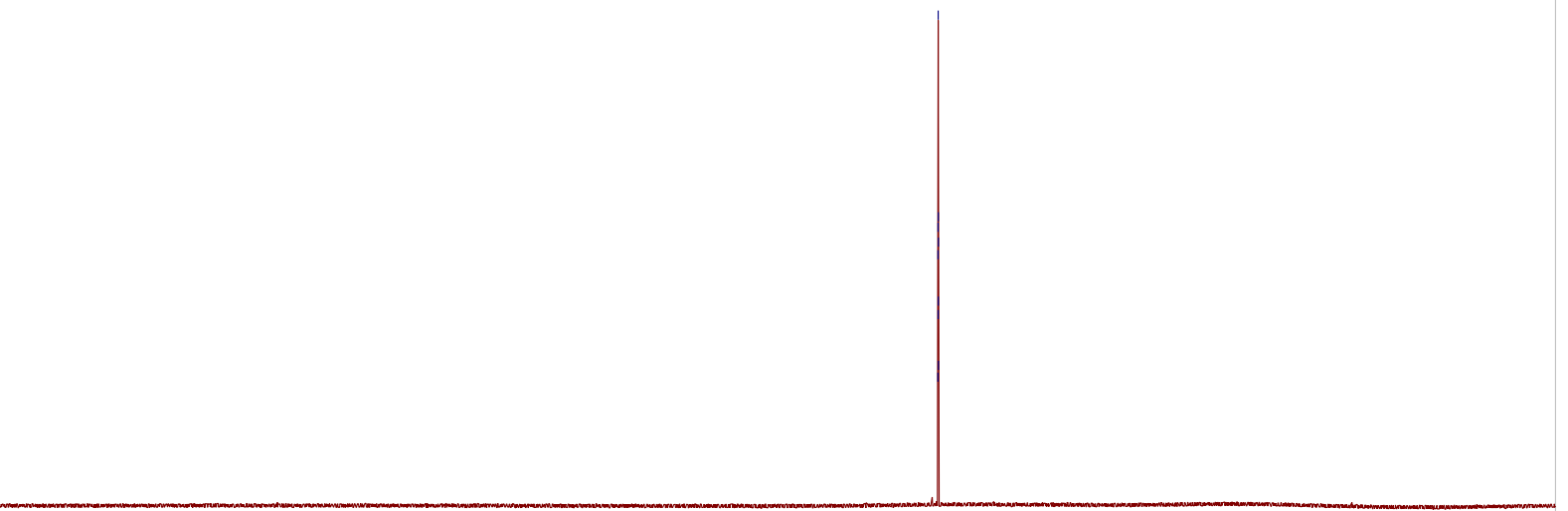

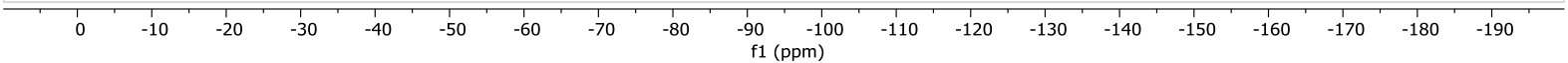

20210611-1526-B400_B.11-9.10.fid

Ref TS2-77C

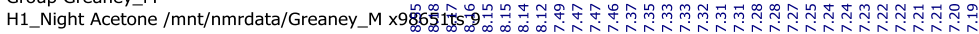<smiles>O=[N+]([O-])c1ccc(Nc2cccc(Br)c2)cc1</smiles>

$2 \mathrm{~g}$

$400 \mathrm{MHz}$
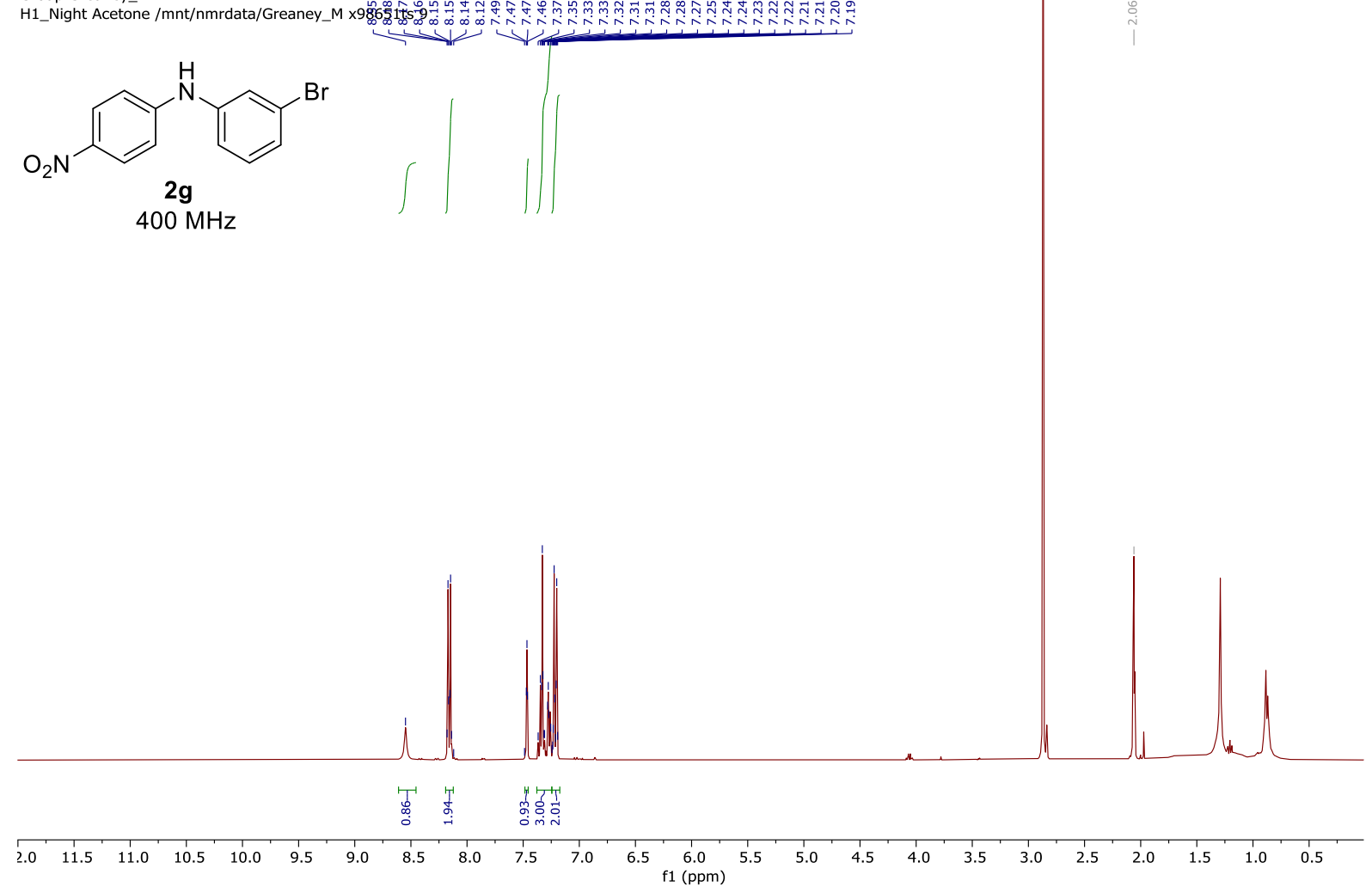
20210611-L526-B400_B.11-9.11.fid

Ref TS2-777

Group_Greaney_M

$\mathrm{H}$

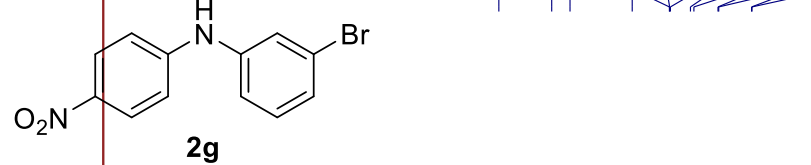

$101 \mathrm{MHz}$

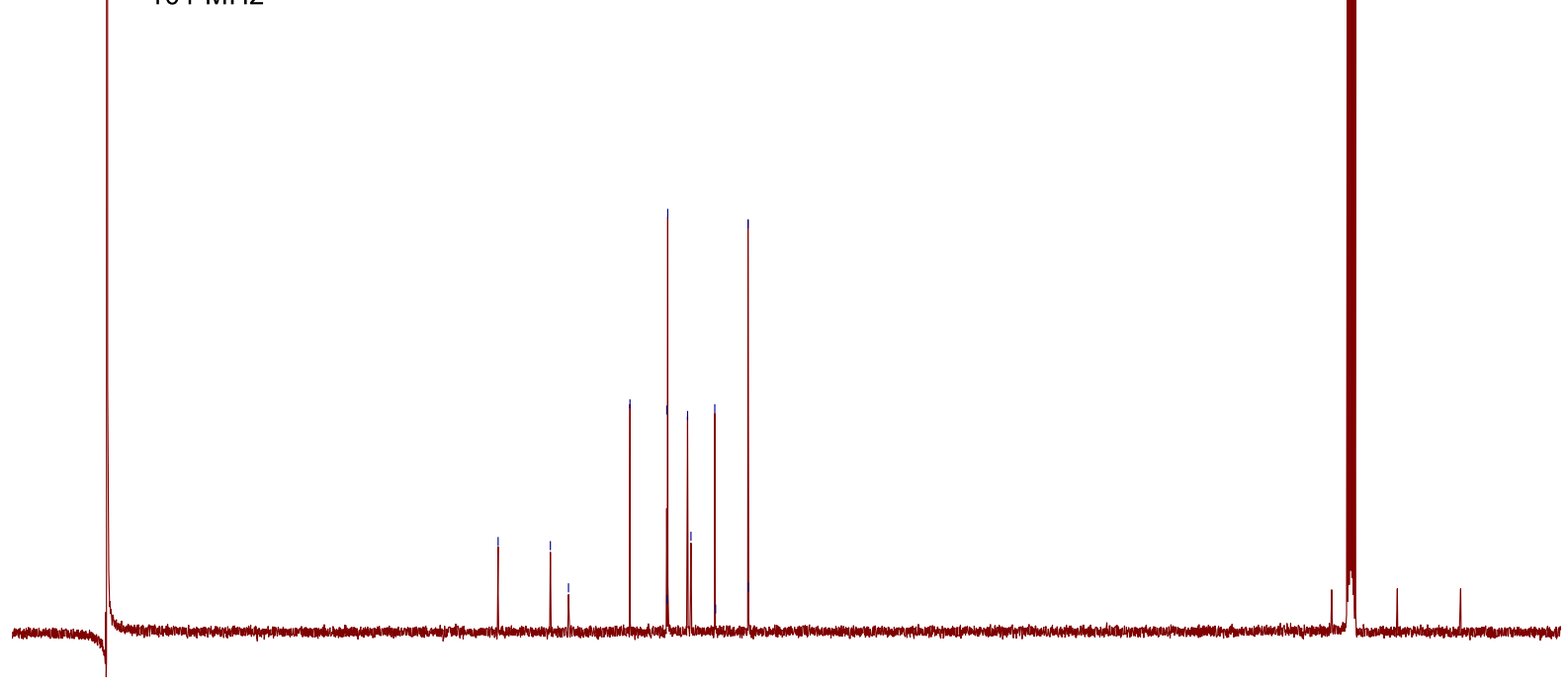

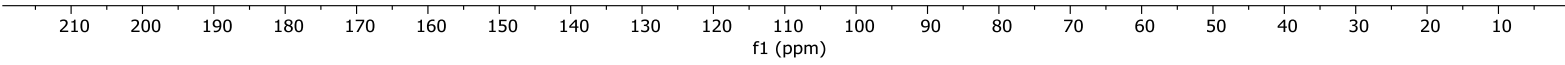

20210611-1527-B400_B.11-56.10.fid

Ref TS2-78C

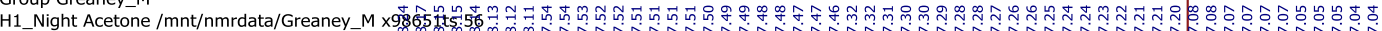<smiles>O=[N+]([O-])c1ccc(Nc2ccccc2F)cc1</smiles>

$2 \mathrm{~h}$

$400 \mathrm{MHz}$
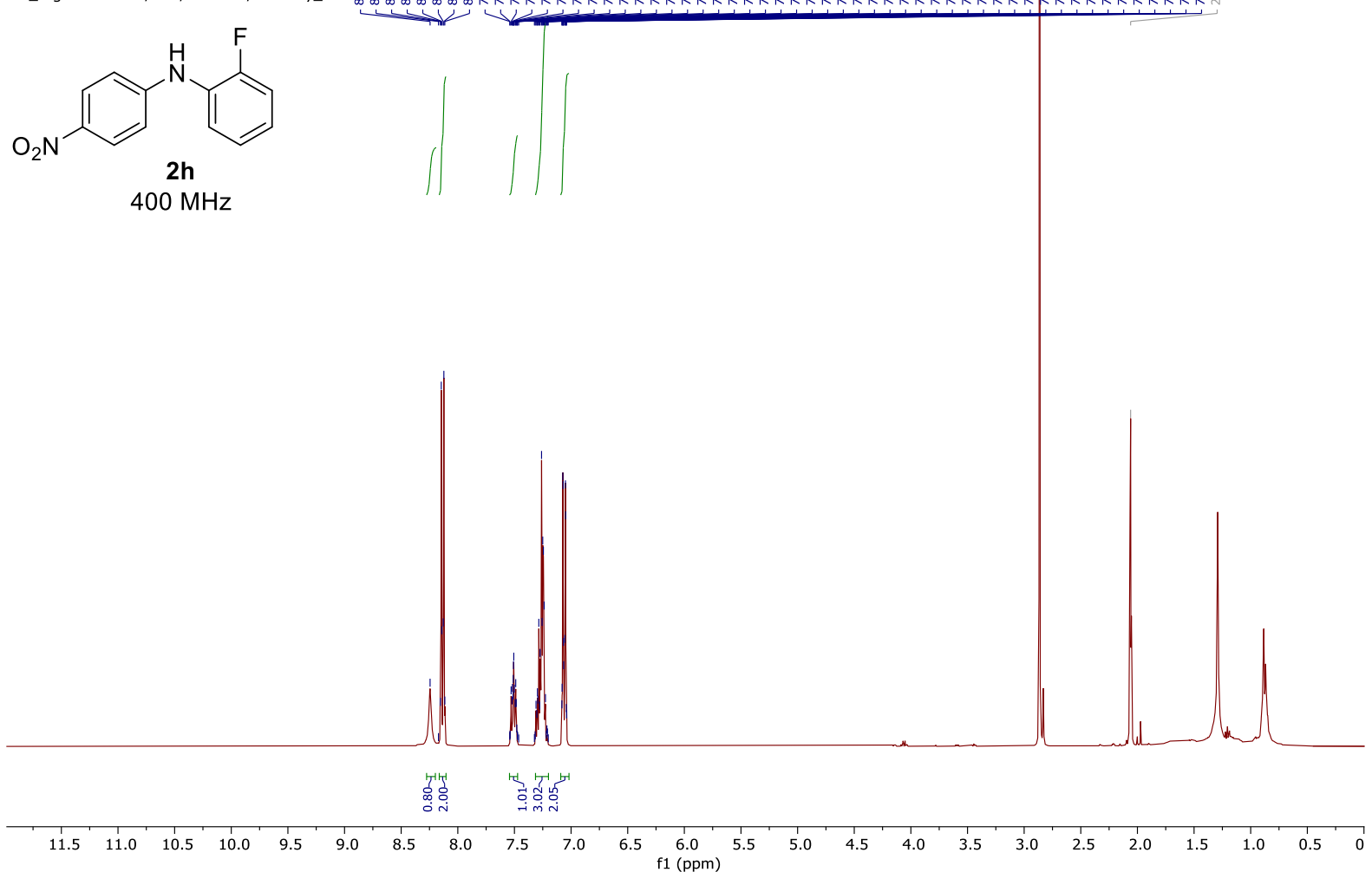
20210611-|527-B400_B.11-56.11.fid

Ref TS2-78C

Group Greaney_M

H F F<smiles>Cc1ccc(Nc2ccccc2F)cc1[N+](=O)[O-]</smiles>

$2 \mathrm{~h}$

$101 \mathrm{MHz}$

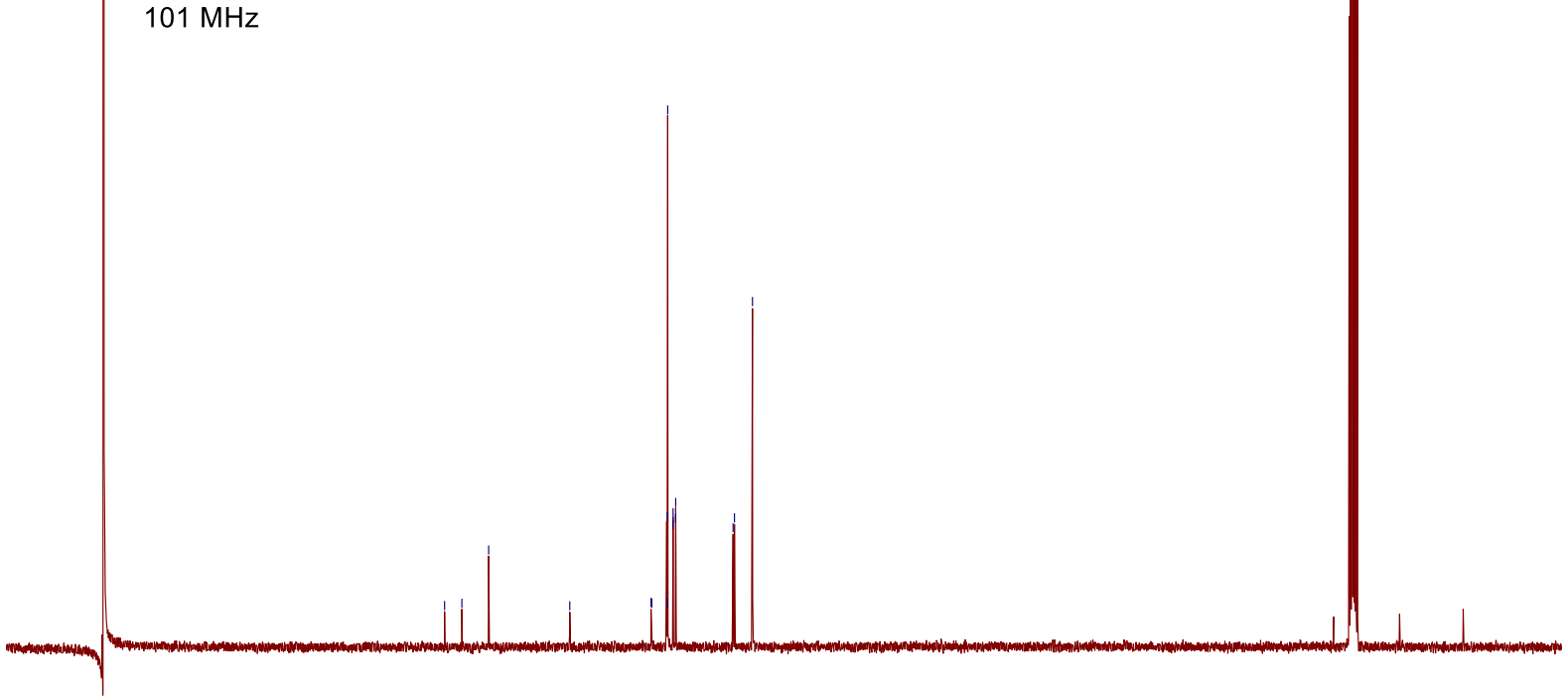

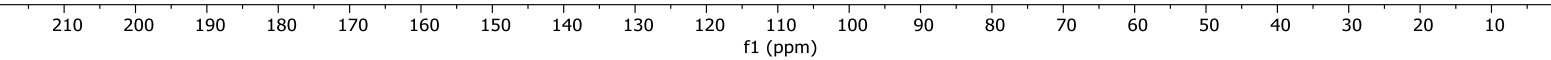

20210611-1527-B400 B.11-56.15 fid

Ref TS2-78C

F19_NoCPD_Night Acetone/mnt/nmrdata/Greaney_M x98651ts 56

(n)

$376 \mathrm{MHz}$
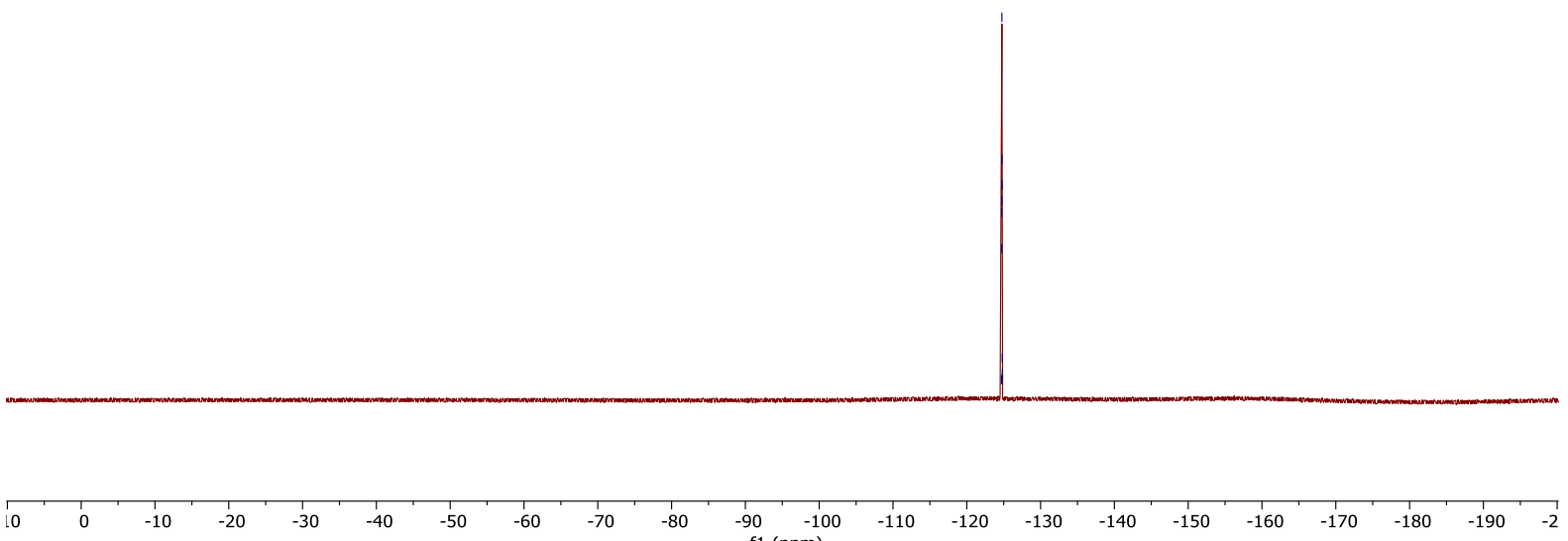

$-40$

$\begin{aligned} & 1 \\ & -60\end{aligned}-70$

$-80$

$\begin{array}{cc}-90 & -100 \\ \mathrm{f} 1(\mathrm{ppm})\end{array}$ 
20210512-1135-B400_B.12-37.10.fid

Ref TS2-56

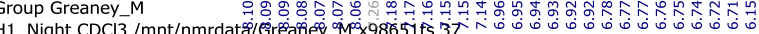<smiles>COc1ccc(Nc2ccc([N+](=O)[O-])cc2)cc1</smiles>

$400 \mathrm{MHz}$
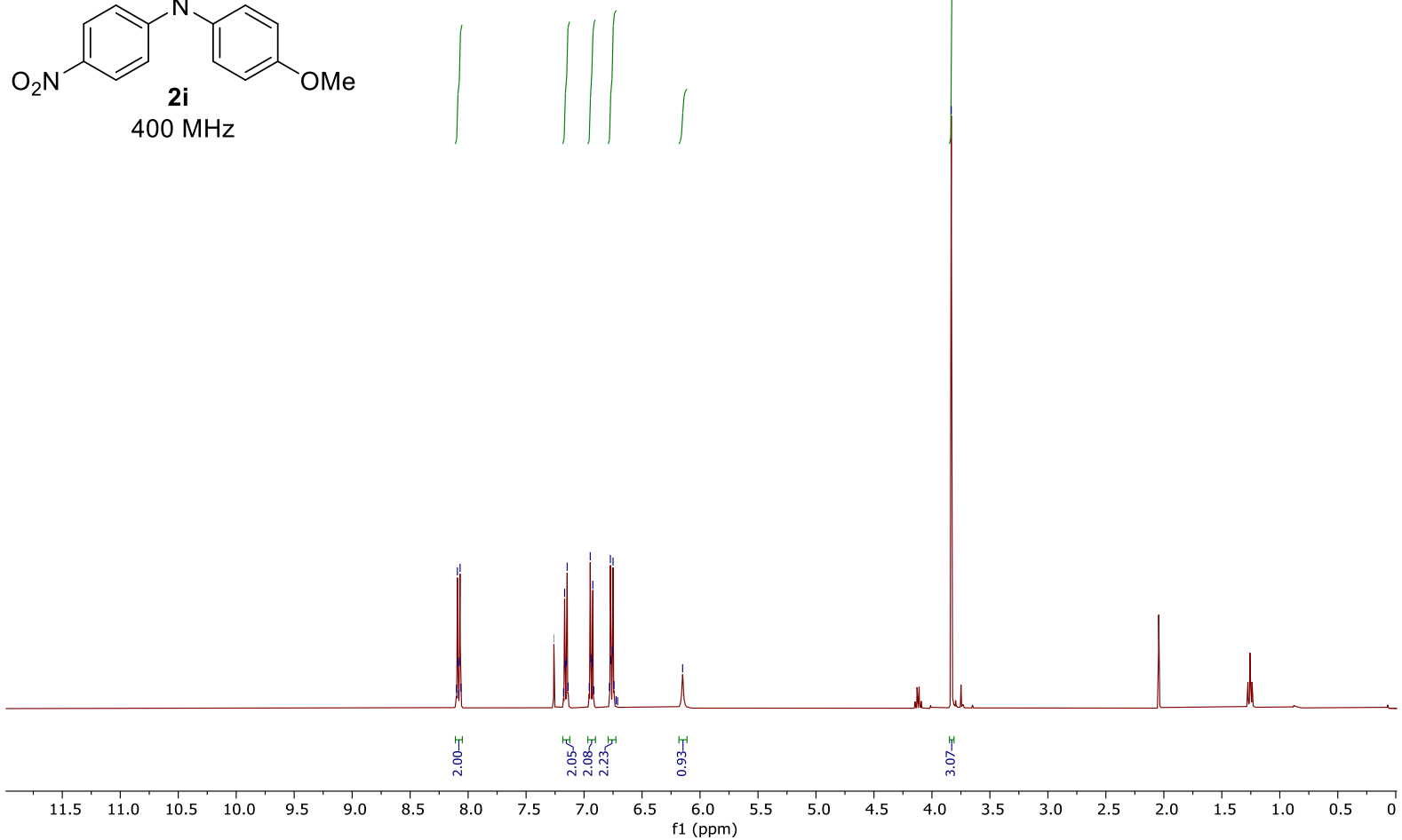

20210512-1135-B400_B.12-37.11.fid

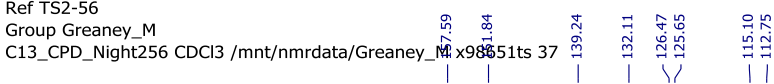<smiles>COc1ccc(Nc2ccc([N+](=O)[O-])cc2)cc1</smiles>

$101 \mathrm{MHz}$

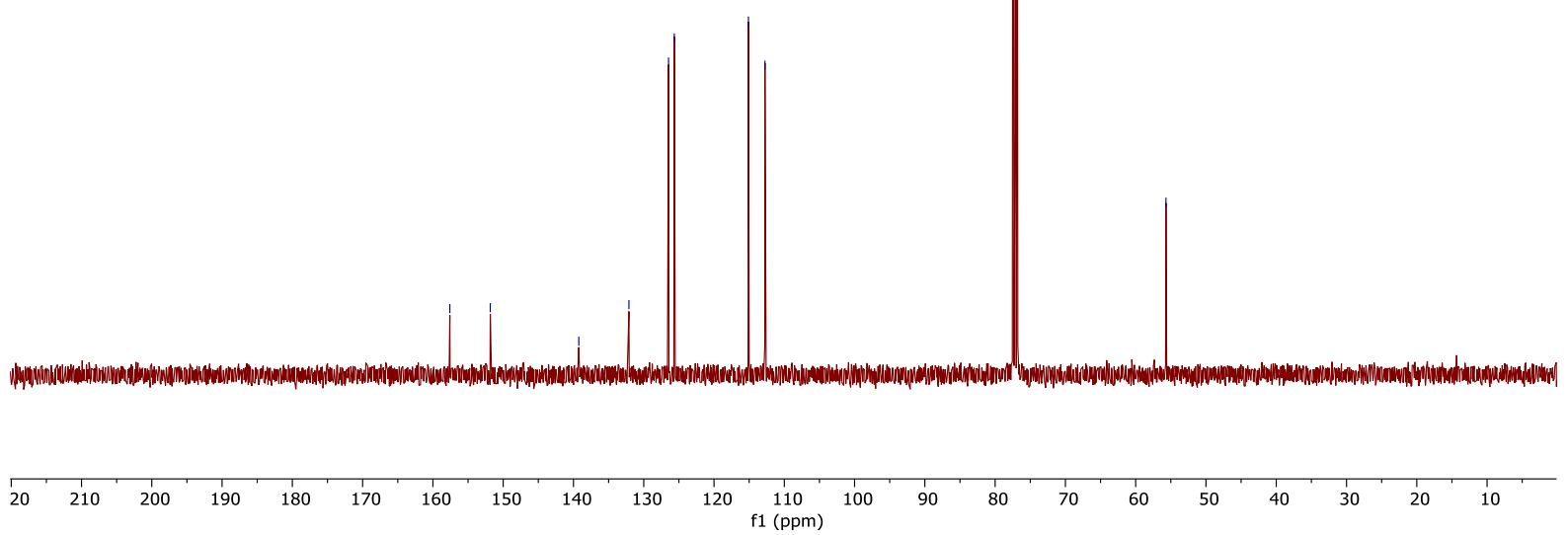


20210823-1152-B500_B.14-28.10.fid

Ref TS2-139FC

Group Greaney_M<smiles>COc1cccc(Nc2ccc([N+](=O)[O-])cc2)c1</smiles>

2

$500 \mathrm{MHz}$
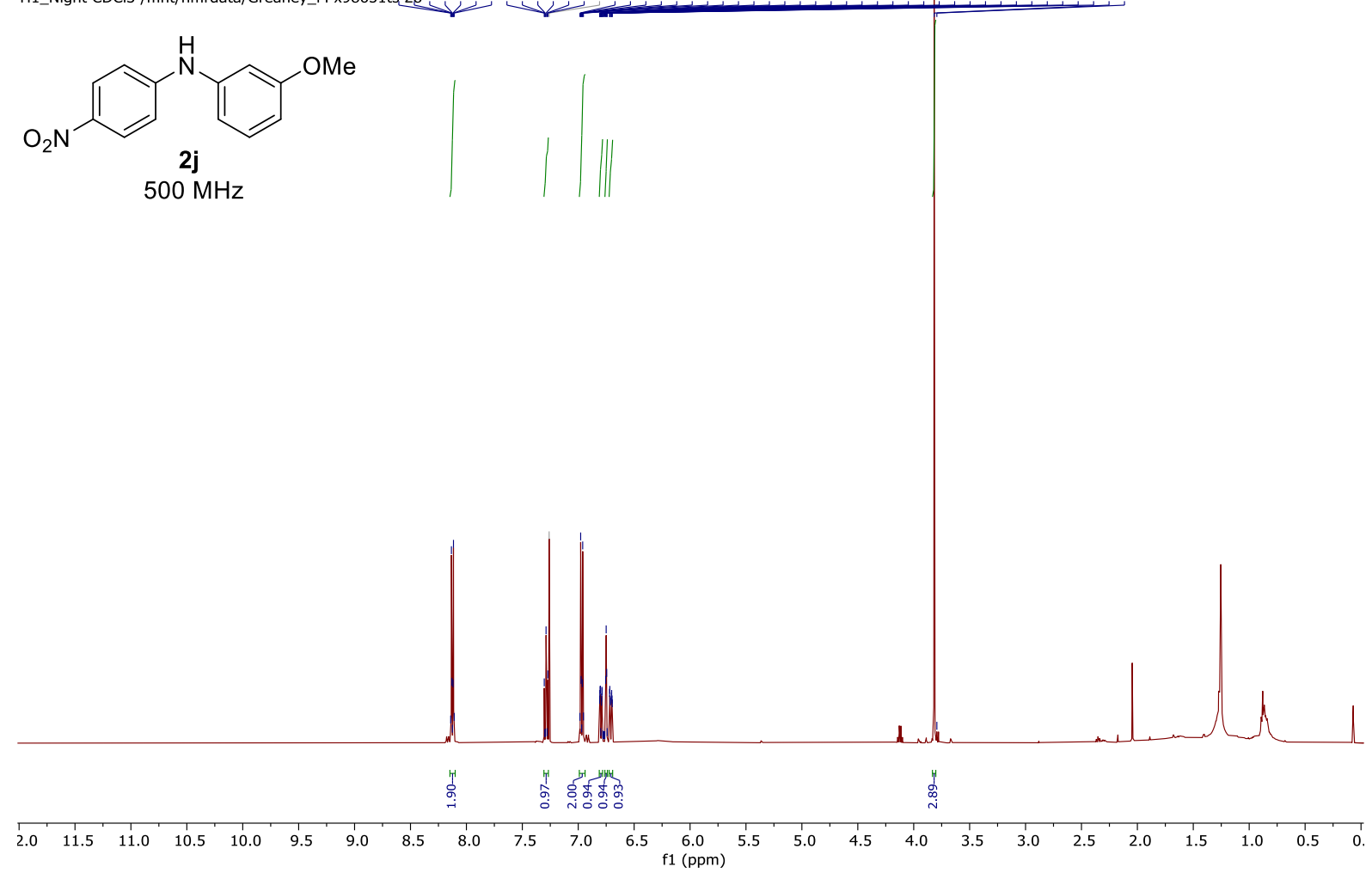

20210823-1152-B500_B.14-28.11.fid

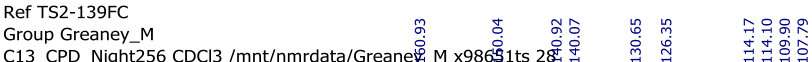

l $\vec{i} \vec{i} \quad \vec{i}$<smiles>COc1cccc(Nc2ccc([N+](=O)[O-])cc2)c1</smiles>

$126 \mathrm{MHz}$

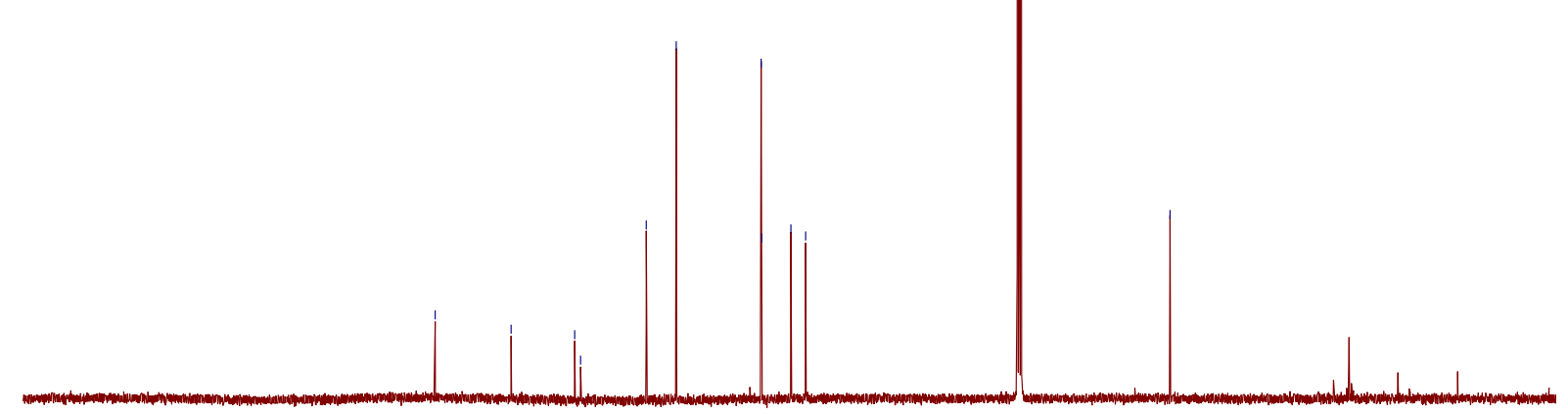

$20210 \quad 200 \quad 190$

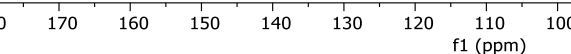

90

10 
20210823-1151-B500_B.14-27.10.fid

Ref TS2-138FC

Group Greaney_M

H1_Night DMSO /mnt/nmrdata/Greaney_M x98651ts 27

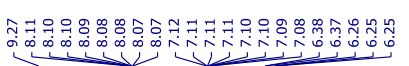<smiles>COc1cc(Nc2ccc([N+](=O)[O-])cc2)cc(OC)c1</smiles>

$500 \mathrm{MHz}$
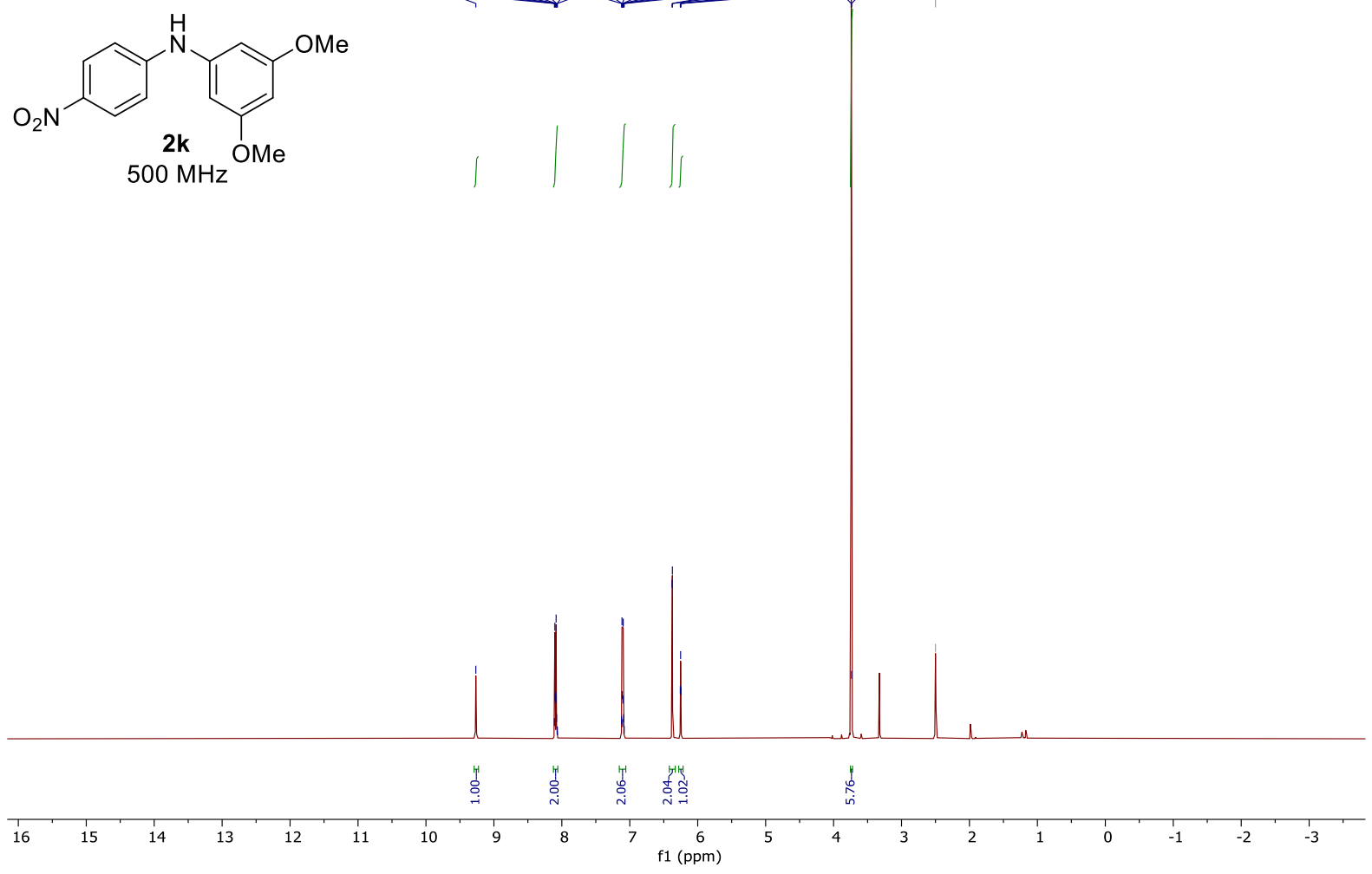

20210823-1151-B500_B.14-27.11.fid

Ref TS2-138FC

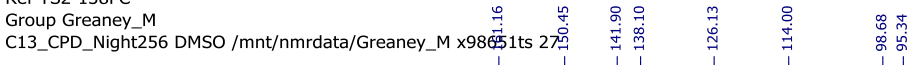<smiles>COc1cc(Nc2ccc([N+](=O)[O-])cc2)cc(OC)c1</smiles>

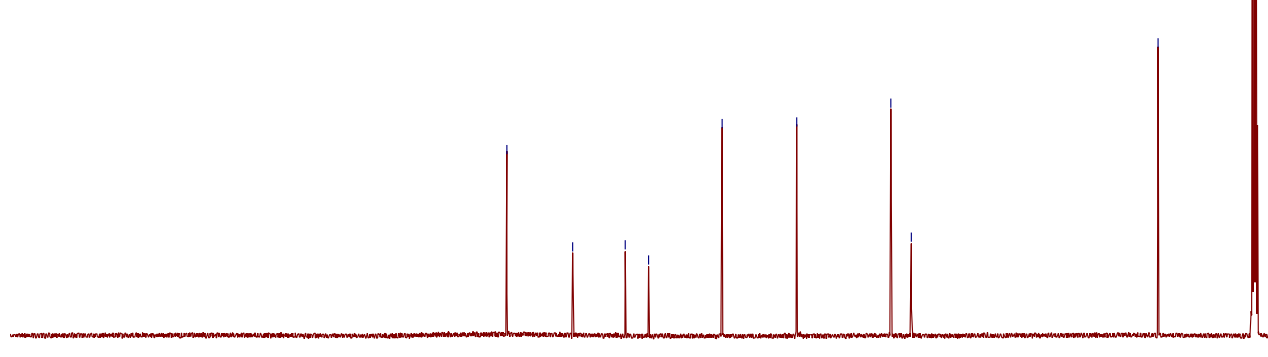

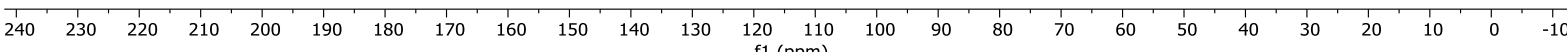


20210526-1550-B400_B.11-37.10.fid

Ref TS2-65C

Group Greaney_M

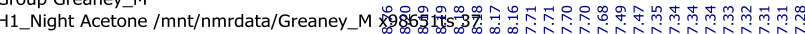<smiles>CC(=O)O[Na]</smiles>
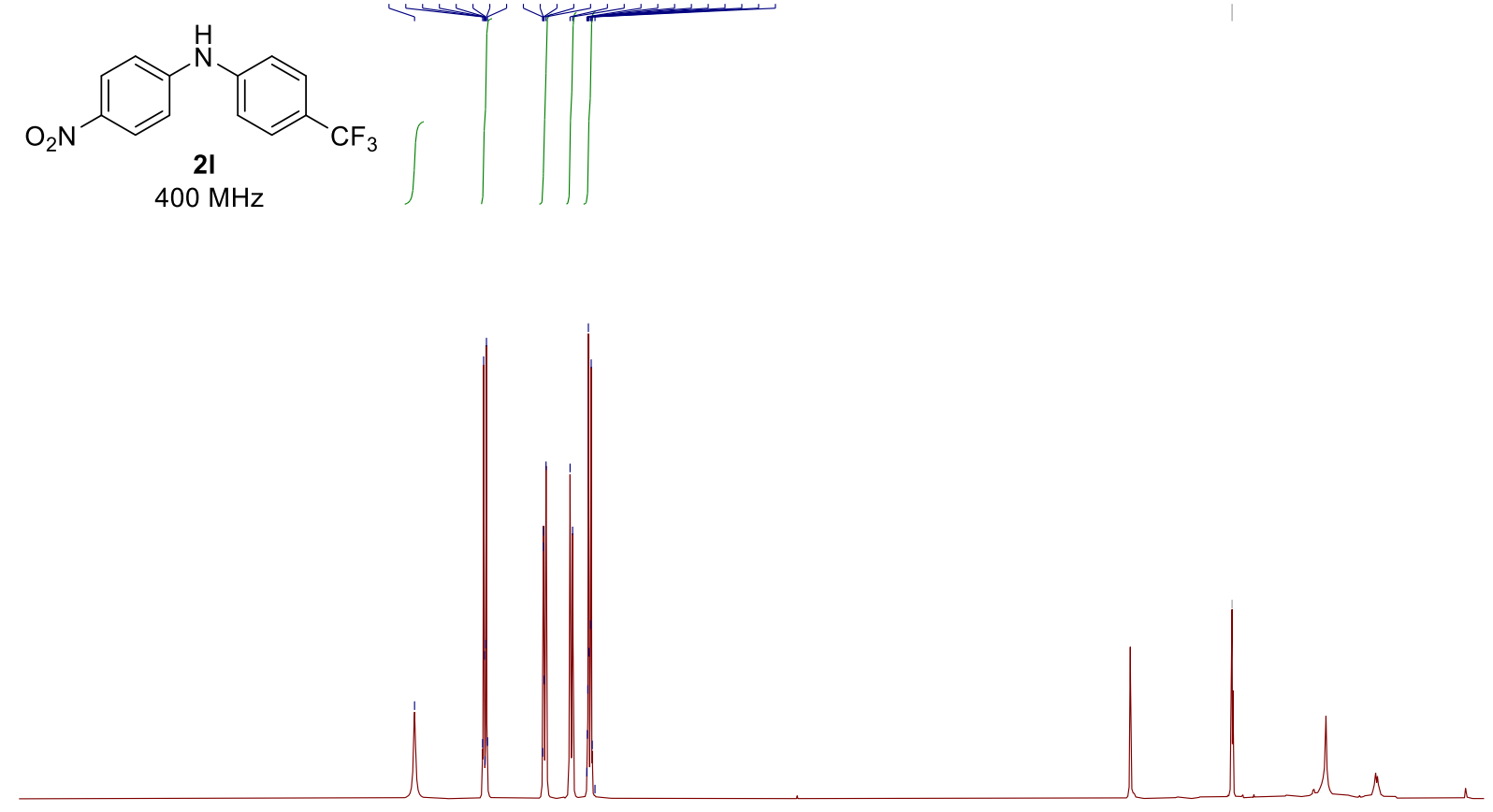

$\begin{array}{llllllllllllllllllllllllll}2.0 & 11.5 & 11.0 & 10.5 & 10.0 & 9.5 & 9.0 & 8.5 & 8.0 & 7.5 & 7.0 & 6.5 & 6.0 & 5.5 & 5.0 & 4.5 & 4.0 & 3.5 & 3.0 & 2.5 & 2.0 & 1.5 & 1.0 & 0.5 & 0\end{array}$

20210526-1550-B400_B.11-37.11.fid

Ref TS2-65C

Group Greaney_M

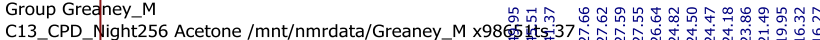

C13_CPD_high256 Acetone/mnt/nmrdata/Greaney_M x986)<smiles>Cc1cc(Nc2ccc(C(F)(F)F)cc2)cc([N+](=O)[O-])c1C</smiles>

$101 \mathrm{MHz}$

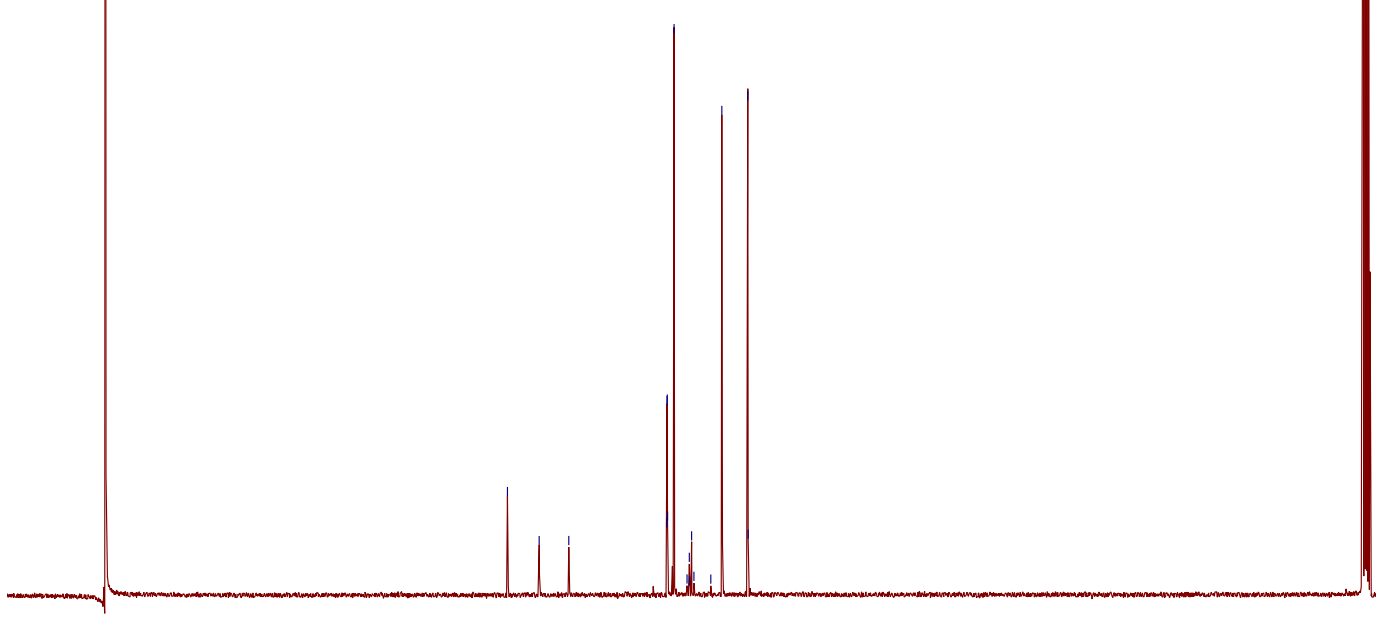

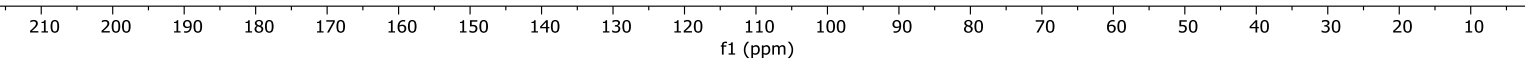


20210526-1550-B400_B.11-37.15.fid

Ref TS2-65C

Group Greaney_M

F19_NoCPD_Day Acetone /mnt/nmrdata/Greaney_M x 98651 ts 37<smiles>O=[N+]([O-])c1ccc(Nc2ccc(C(F)(F)F)cc2)cc1</smiles>

$376 \mathrm{MHz}$

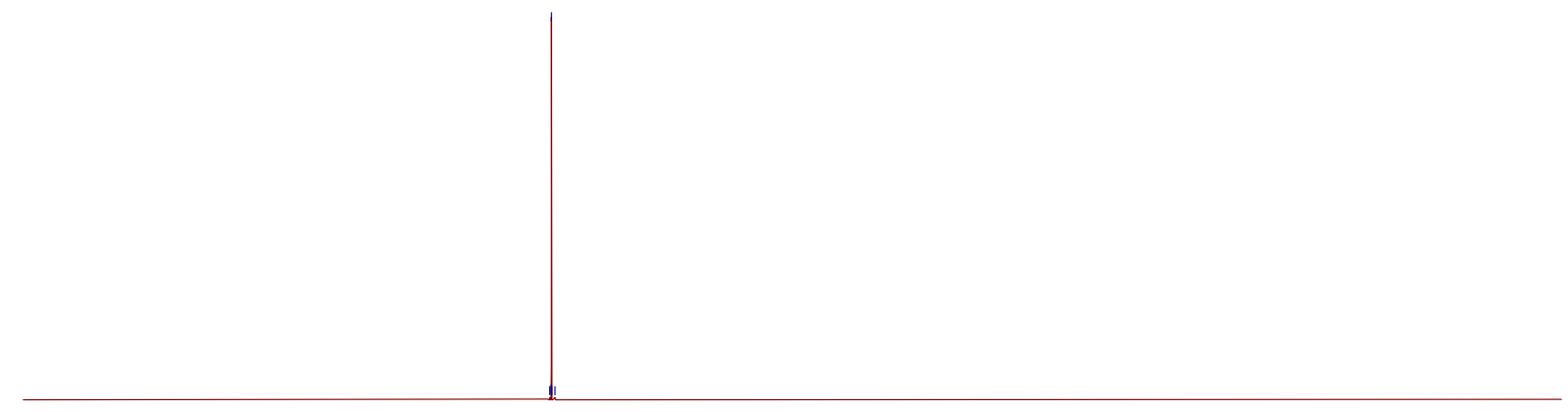

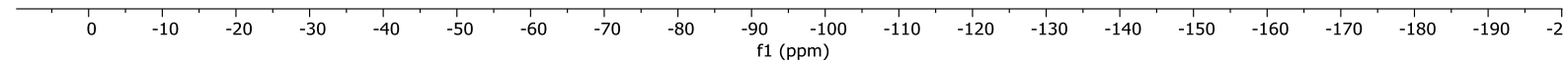

20210625-1523-B400_B.11-50.10.fid

Ref TS2-88C

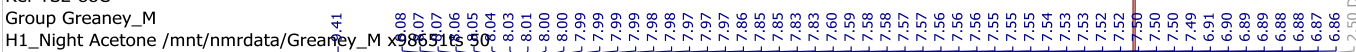<smiles>O=[N+]([O-])c1ccc(Nc2cccc3ccccc23)cc1</smiles>

$400 \mathrm{MHz}$
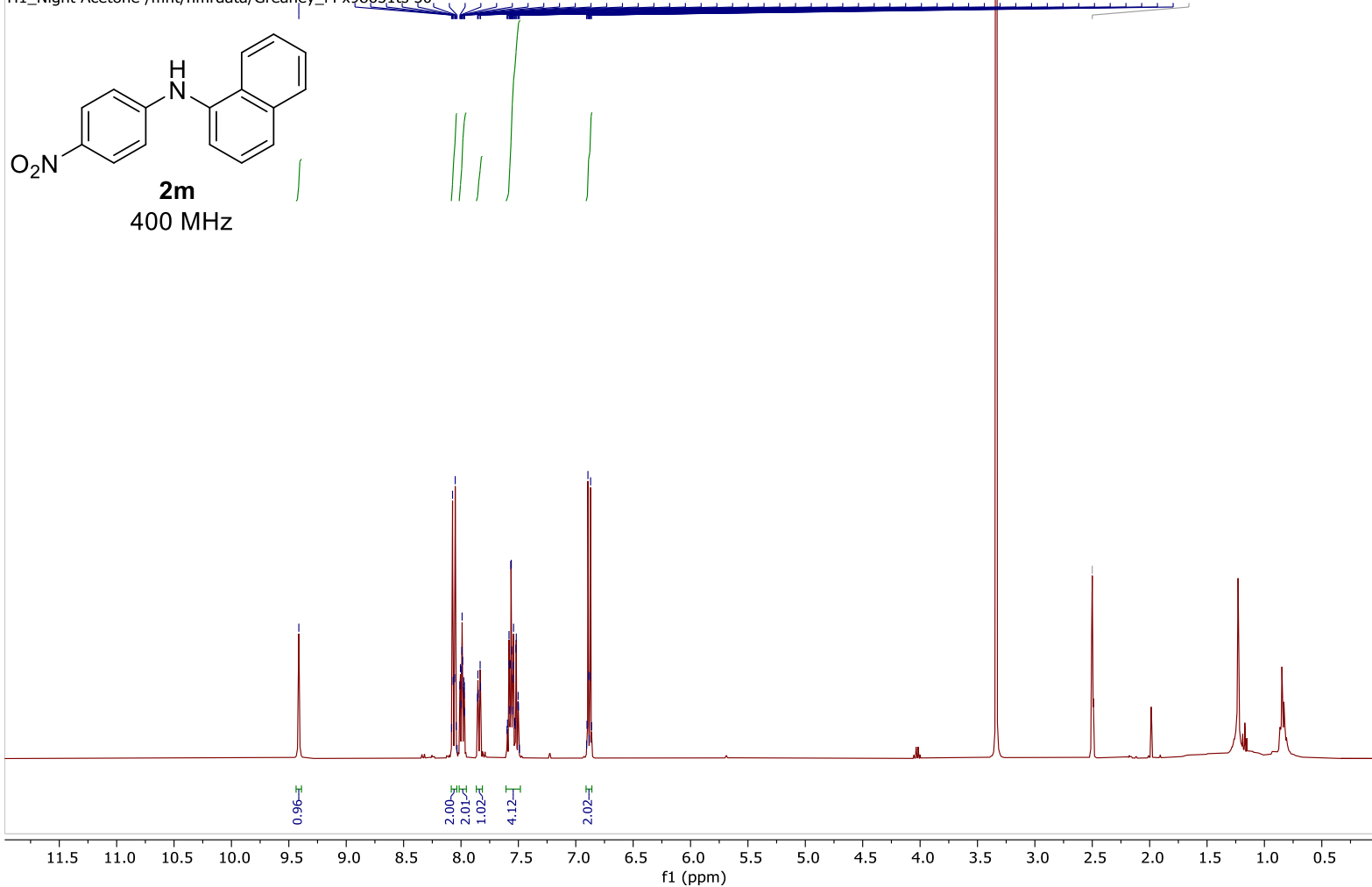
20210625-1523-B400_B.11-50.11.fid

Ref TS2-88C

Group Greaney_M<smiles>O=[N+]([O-])c1ccc(Nc2cccc3ccccc23)cc1</smiles>

$2 \mathrm{~m}$

$101 \mathrm{MHz}$
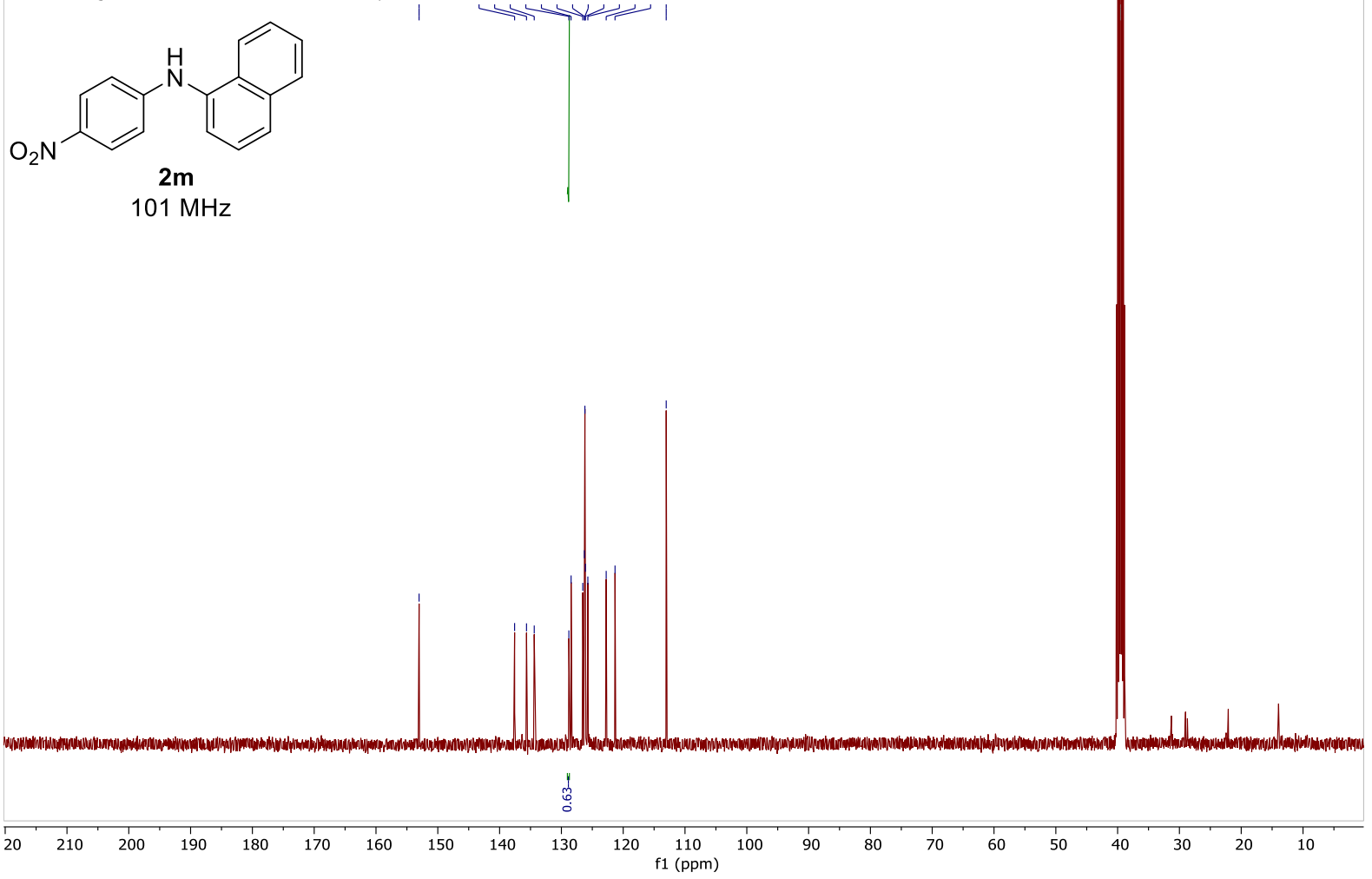

20210723-1806-B500_B.14-35.10.fid

Ref TS2-102FC

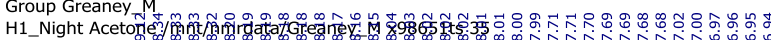<smiles>O=[N+]([O-])c1ccc(Nc2ccccn2)cc1</smiles>

$500 \mathrm{MHz}$
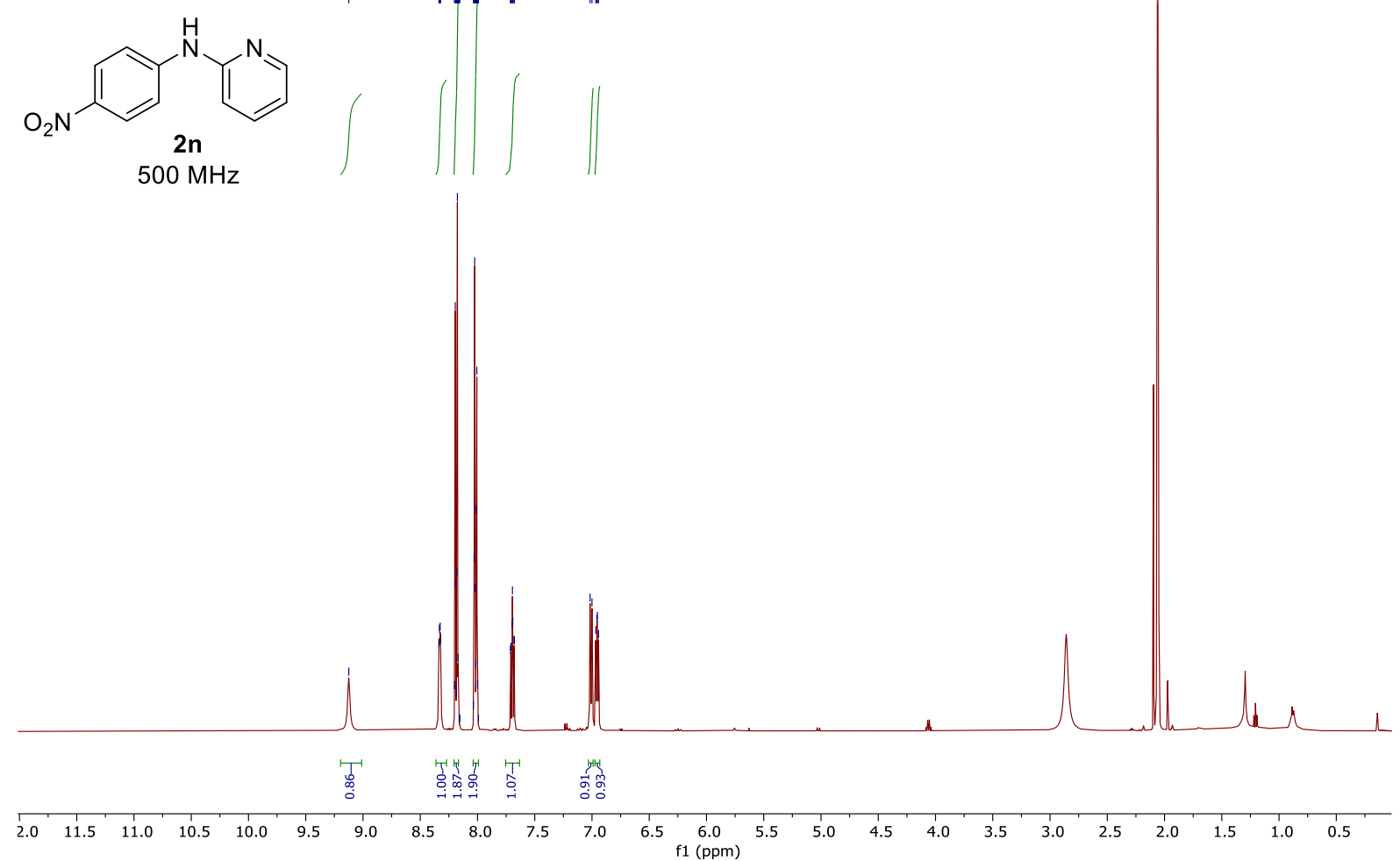


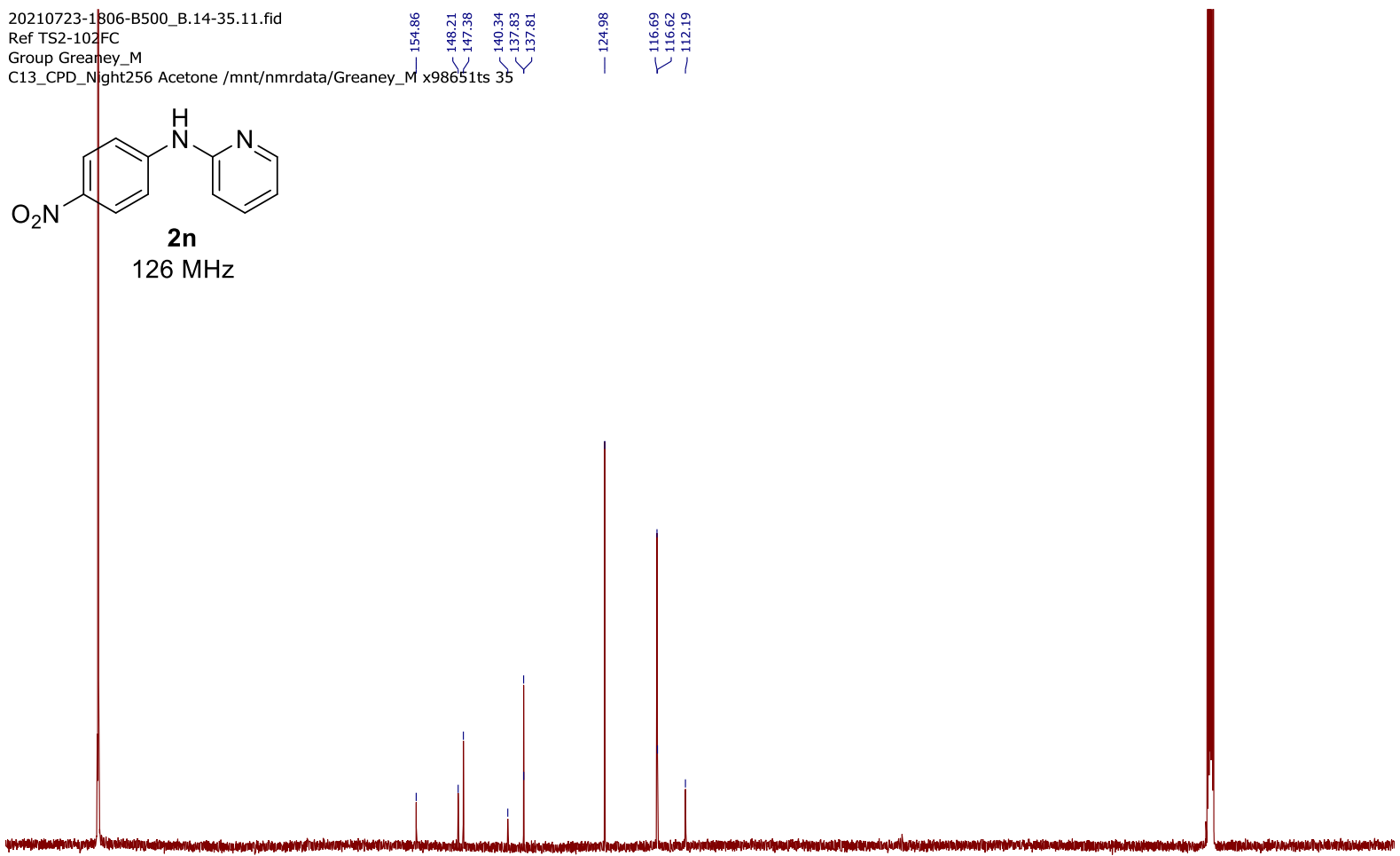

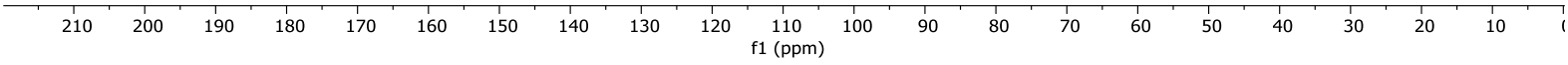

20210728-1448-B400_B.11-41.10.fid

Ref TS2-115FC

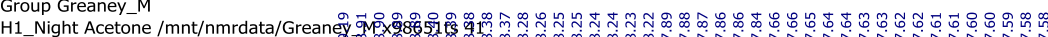

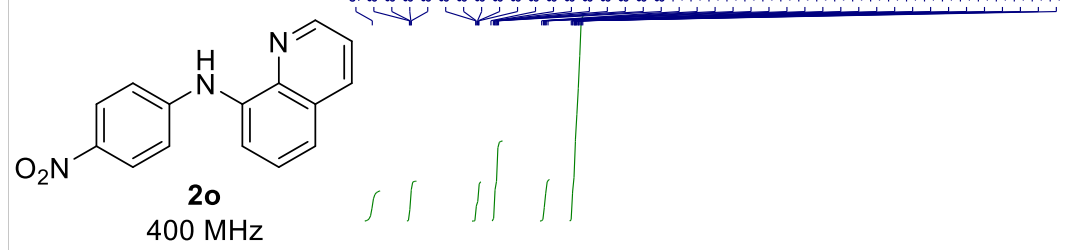

$400 \mathrm{MHz}$

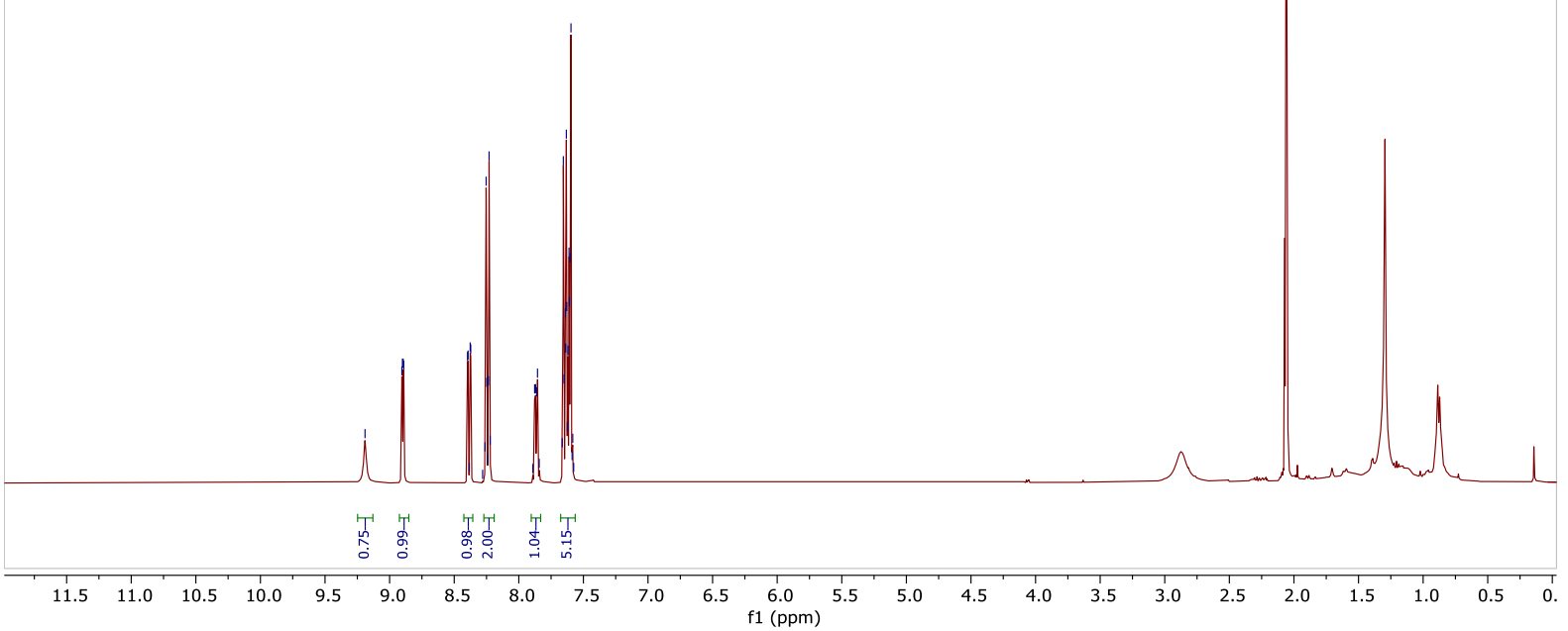


20210728-4448-B400_B.11-41.11.fid

Ref TS2-115FC

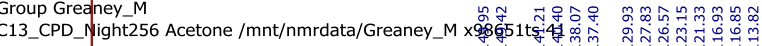

$\mathrm{N}$ र रा। रा।

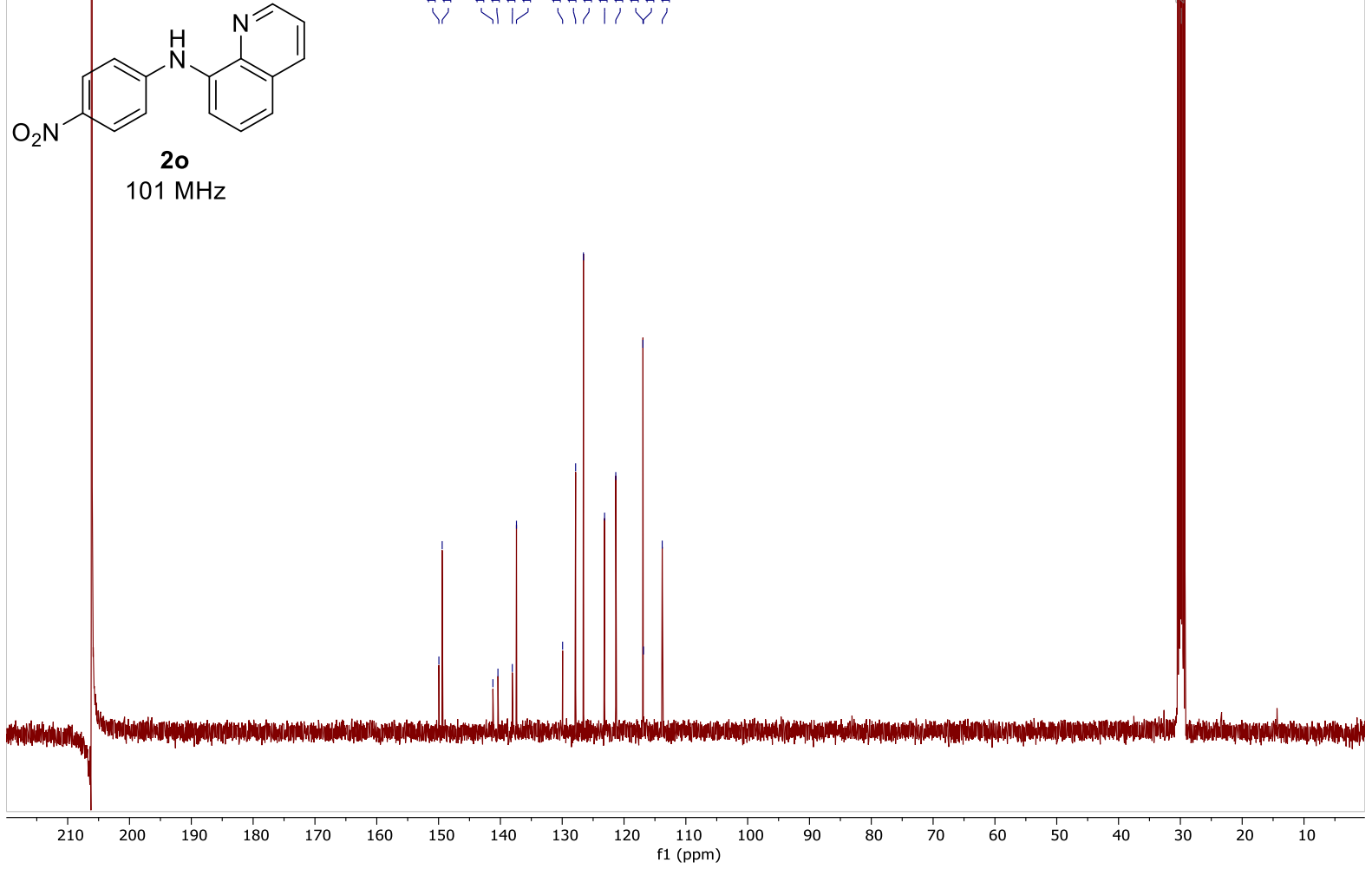

20210621-1145-B500_B.14-21.10.fid

Ref TS2-85C

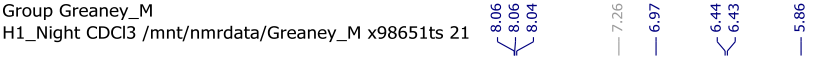<smiles>Cc1cc(C)c(Nc2ccc([N+](=O)[O-])cc2)c(C)c1</smiles>

$2 p$

$500 \mathrm{MHz}$

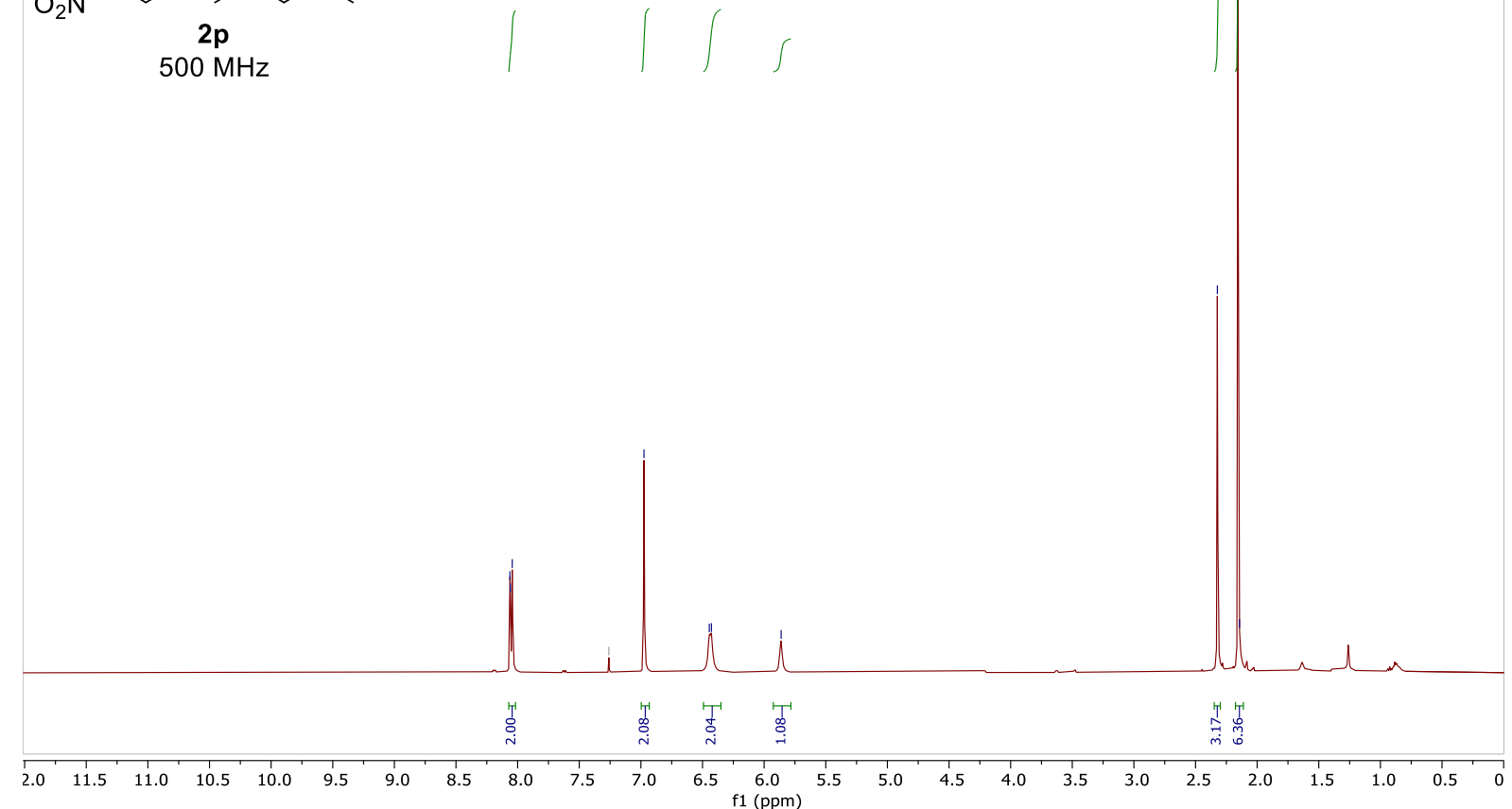



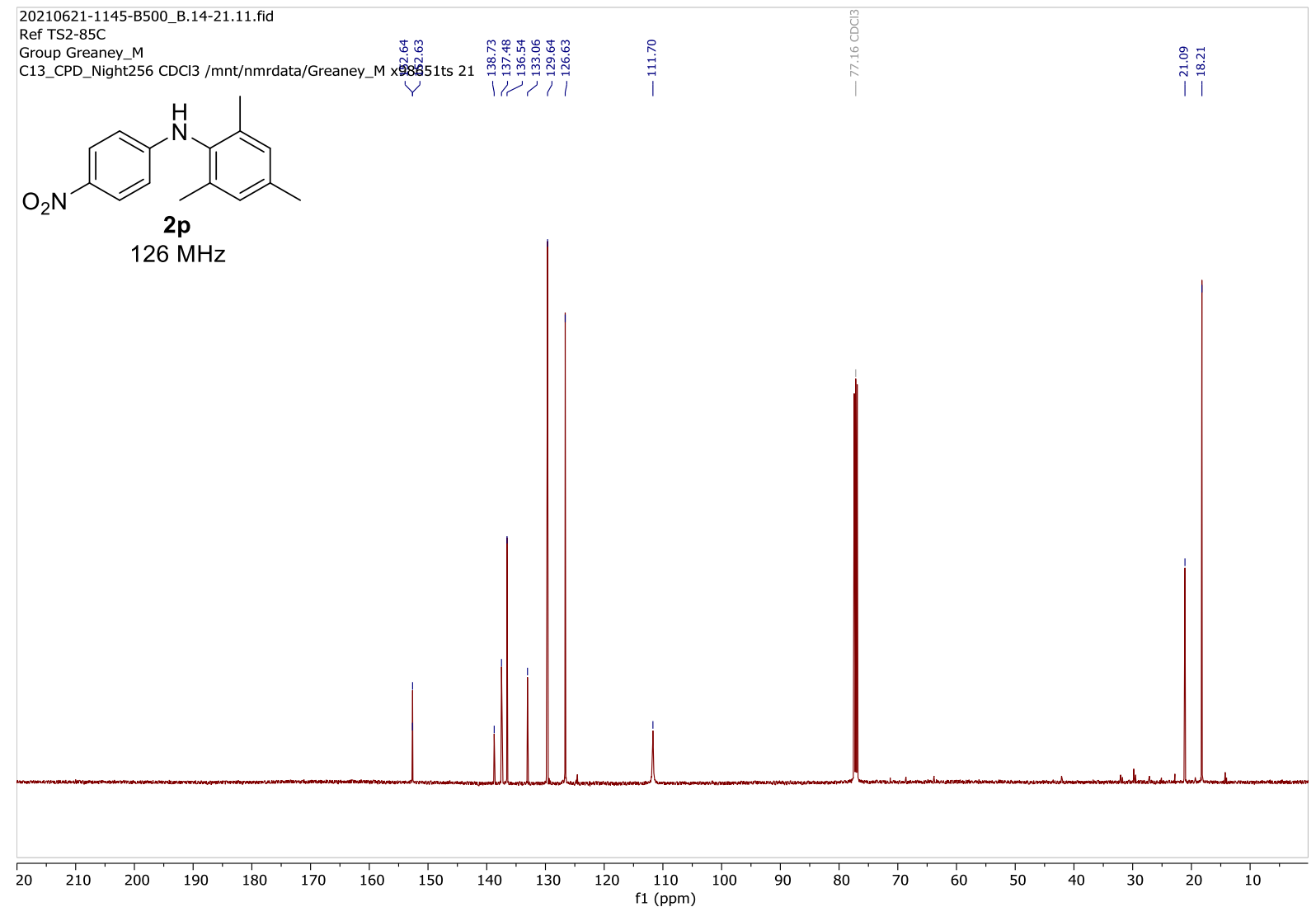

20211001-1506-B400_B.11-57.10.fid

Ref TS3-4FC

Group Greaney_M

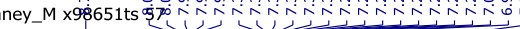<smiles>CC(C)c1cccc(P(N)(=O)OCc2ccc([N+](=O)[O-])cc2)c1Nc1ccccc1</smiles>

$400 \mathrm{MHz}$
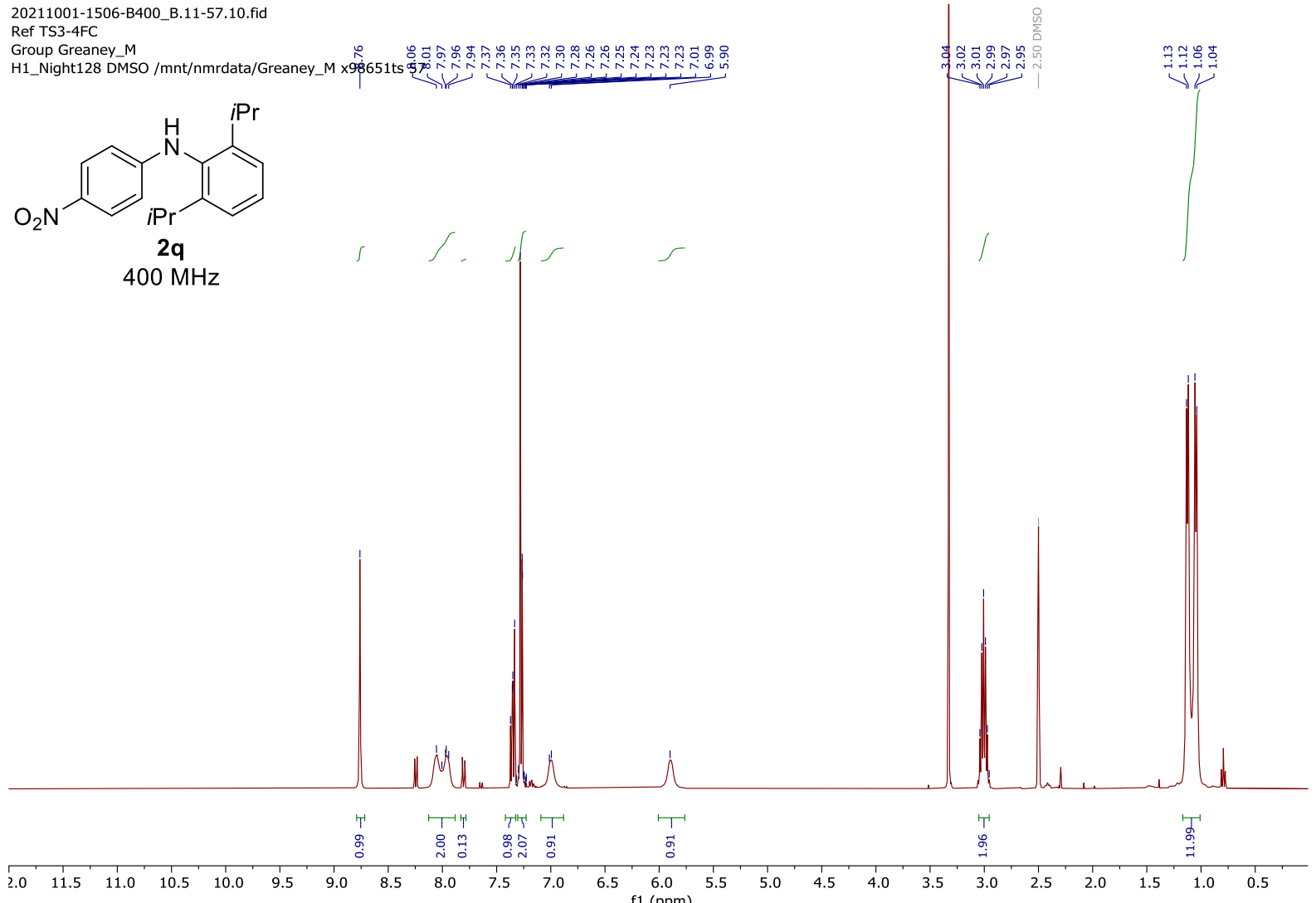
20211001-1506-B400_B.11-57.11.fid

Ref TS3-4FC

Group Greaney_M

(1)

2 q

$101 \mathrm{MHz}$

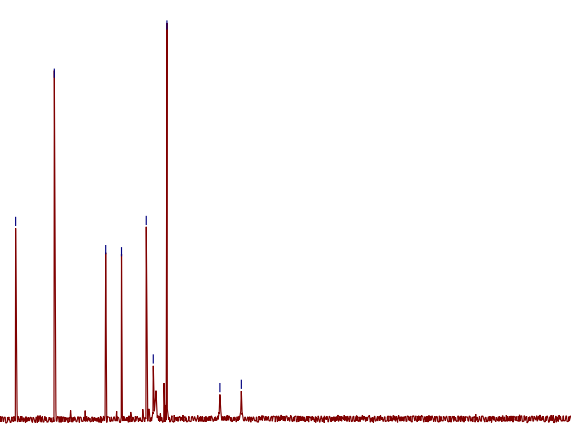

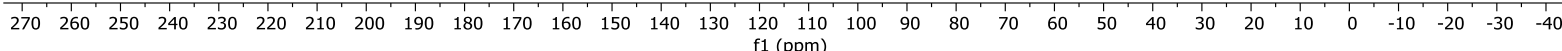

20210816-1120-B500_B.14-11.10.fid

Ref TS2-128FC

H1_Night DMSO /mnt/nmrdata/Greaney_M x98651ts 11

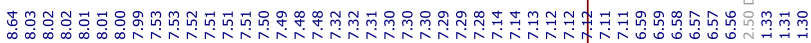

(N)

$2 r$

$500 \mathrm{MHz}$
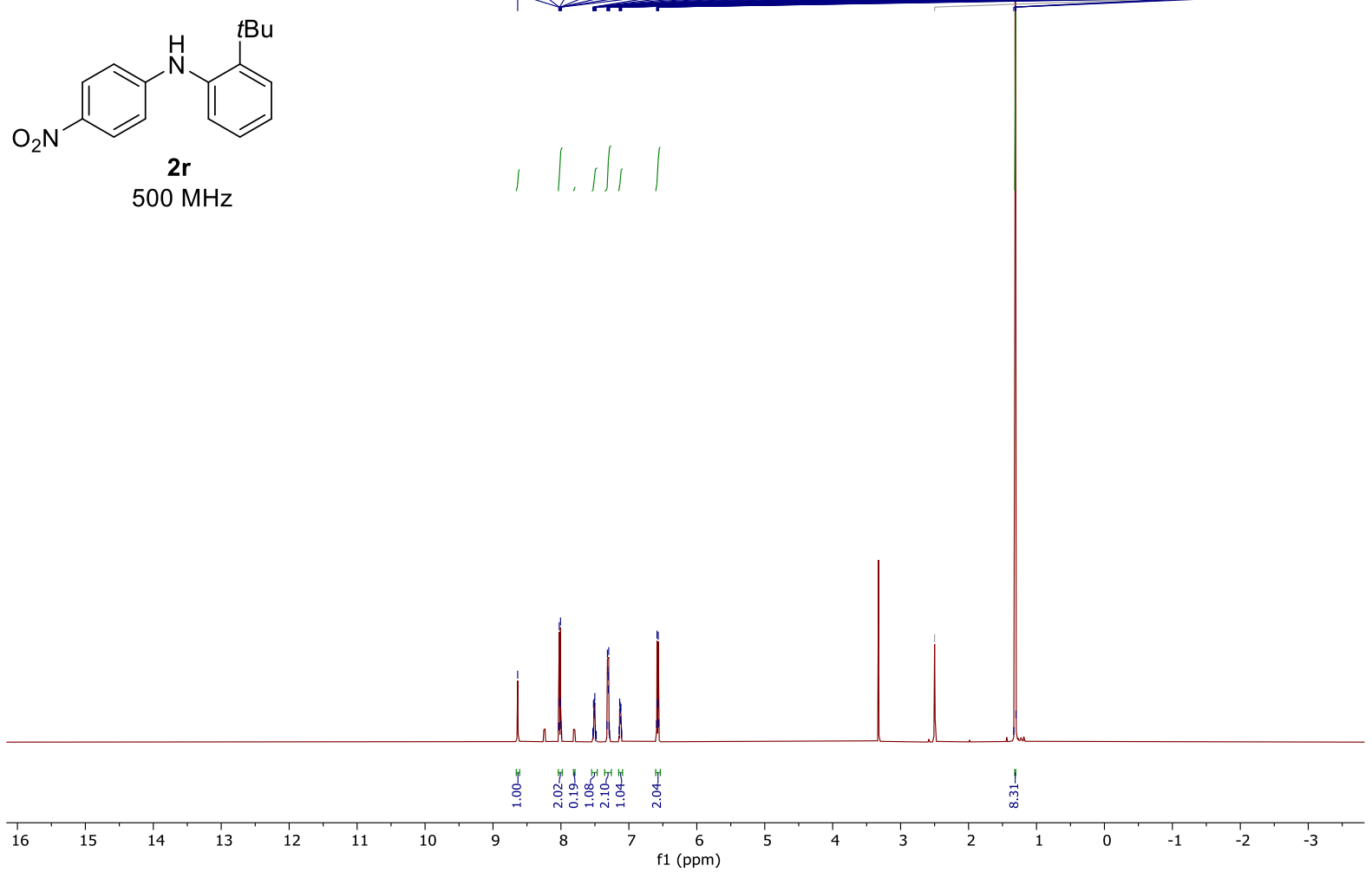
20210816-1120-B500_B.14-11.11.fid

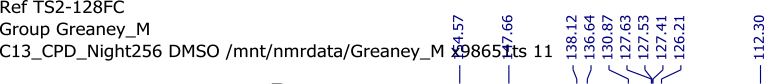

Group Greaney_M<smiles>CC(C)(C)c1ccccc1Nc1ccc([N+](=O)[O-])cc1</smiles>

$126 \mathrm{MHz}$

$\mathrm{Bu}$

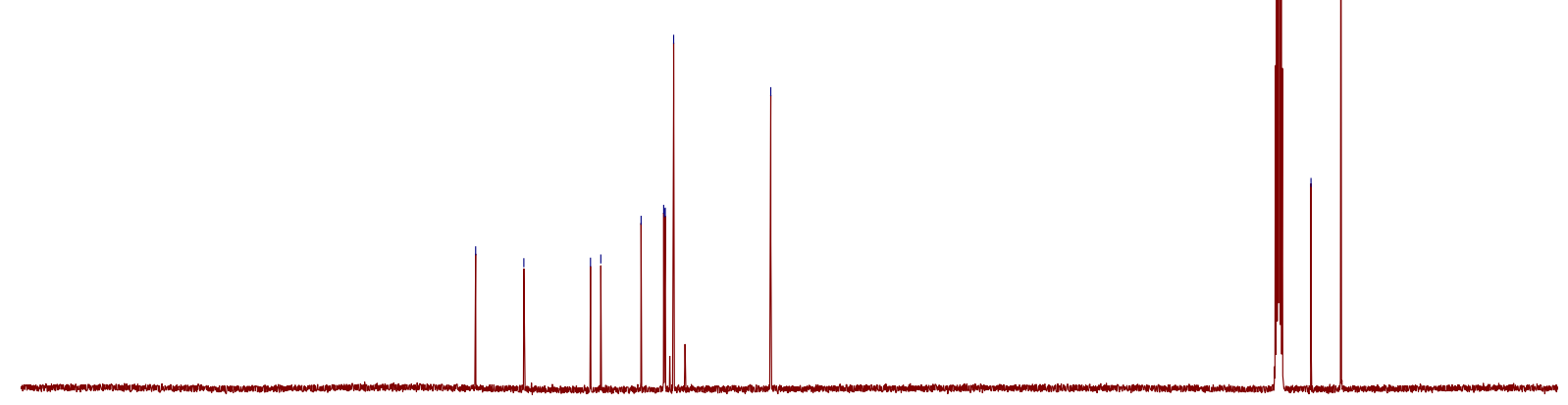

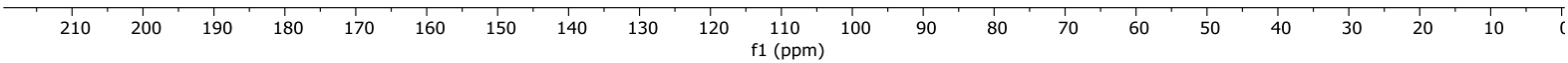

20210913-1458-B400_B.14-49.10.fid

Ref TS2-159FC

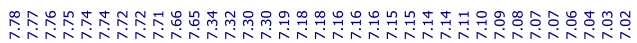

H1_Night CDCl3/mnt/nmrdata/Greaney_M X98651ts 49<smiles>COc1cc([N+](=O)[O-])ccc1Nc1ccccc1</smiles>

$400 \mathrm{MHz}$

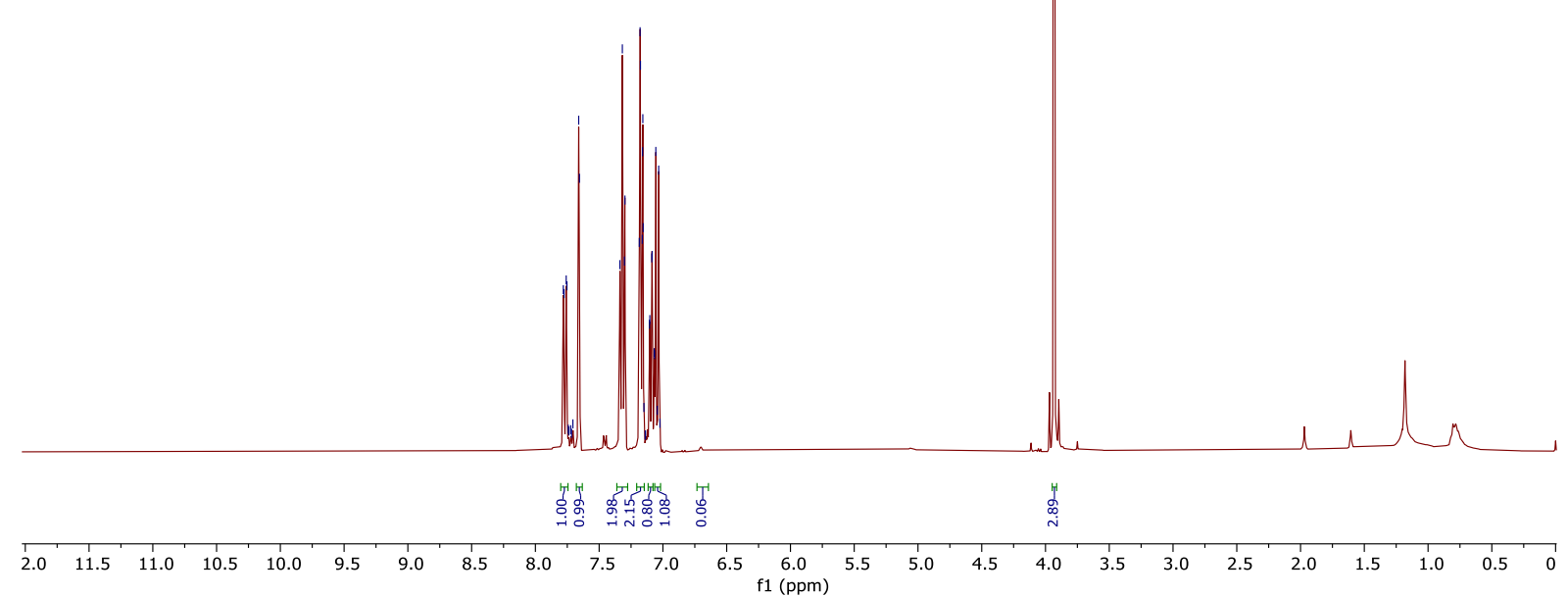


20210913-1458-B400_B.14-49.12.fid

Ref TS2-159FC

Group Greaney_M

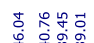

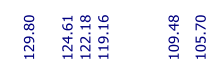

C13_CPD_Night256 CDCl3/mnt/nmrdata/Greaney_M x98651t得, 48

$\mathrm{O}_{2} \mathrm{~N}$<smiles>COc1cc(C)ccc1Nc1ccccc1</smiles>

2s

$101 \mathrm{MHz}$

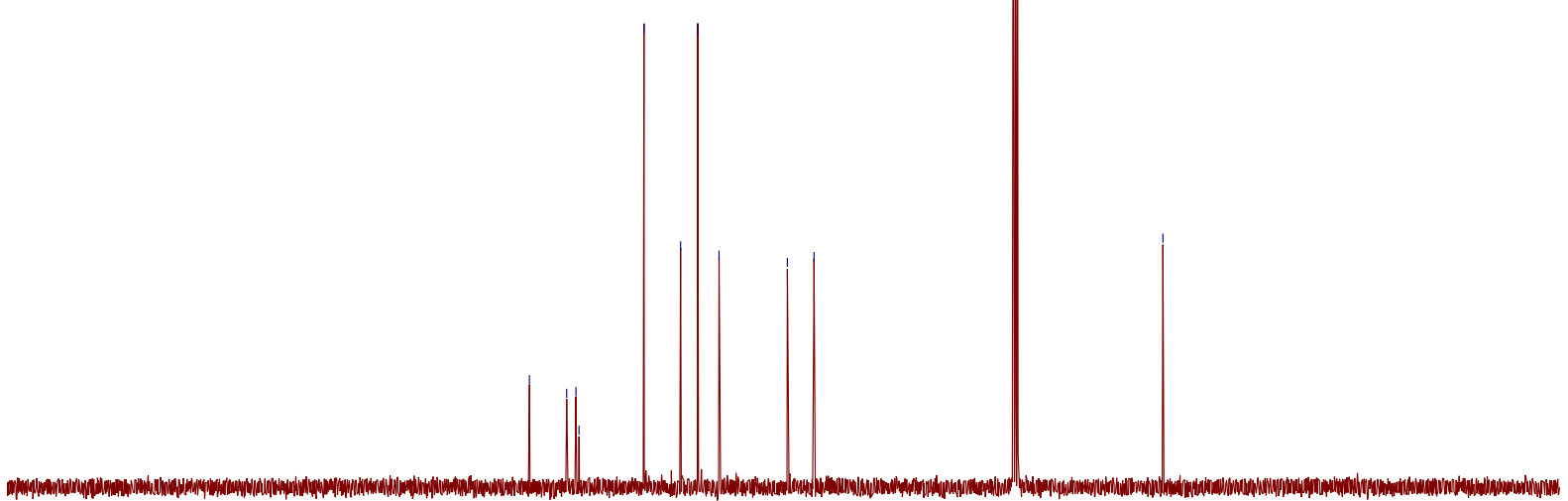

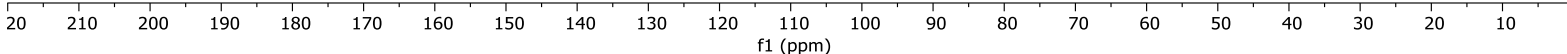

20210917-1438-B500_B.14-52.10.fid

Ref TS2-178FC

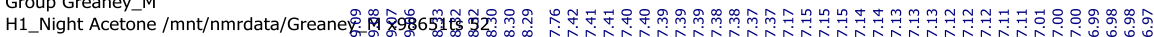

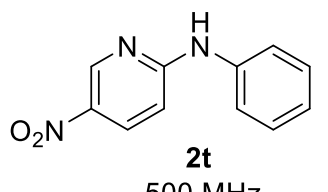

$500 \mathrm{MHz}$
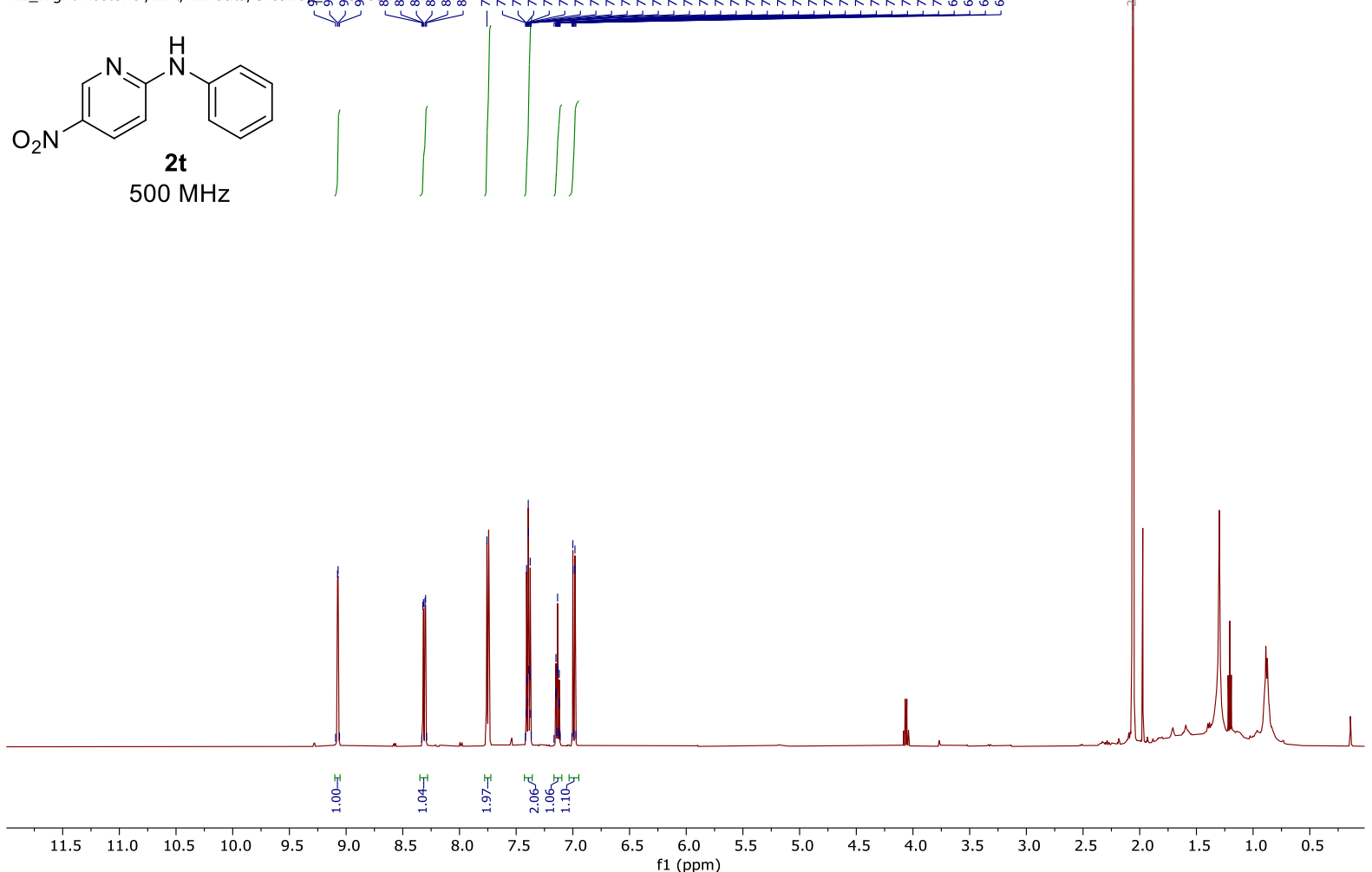
20210917-1438-B500_B.14-52.11.fid

Ref TS2-173FC

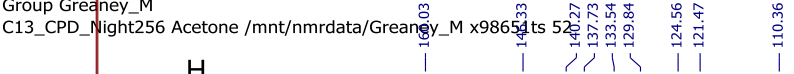<smiles>[R4][R4]([X])([R])C</smiles>

$126 \mathrm{MHz}$

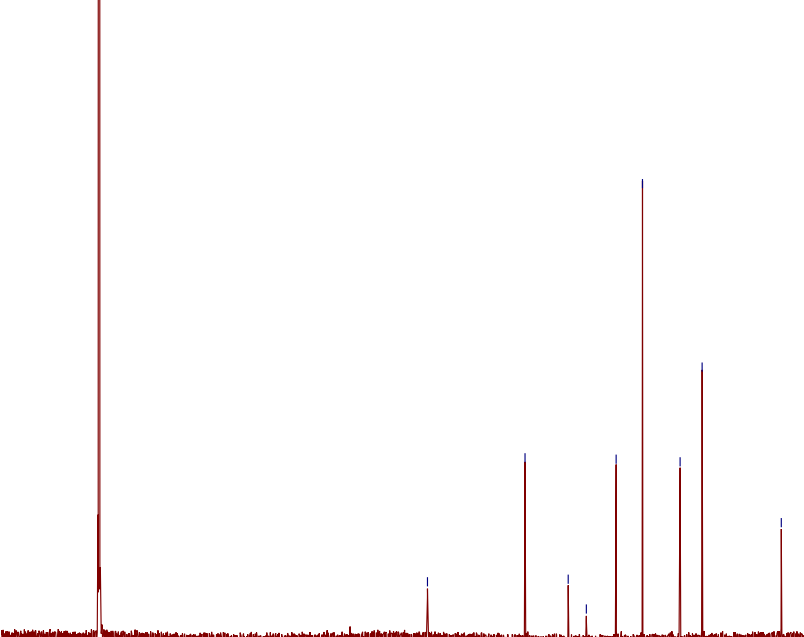

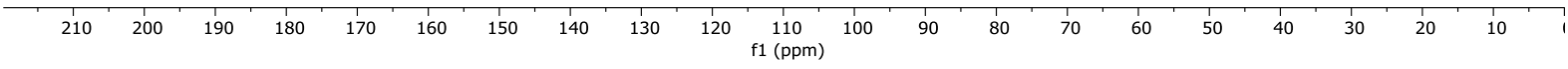

20210329-1732-B500_B.14-9.10.fid

Ref TS2-15

Group Greaney_M<smiles></smiles>
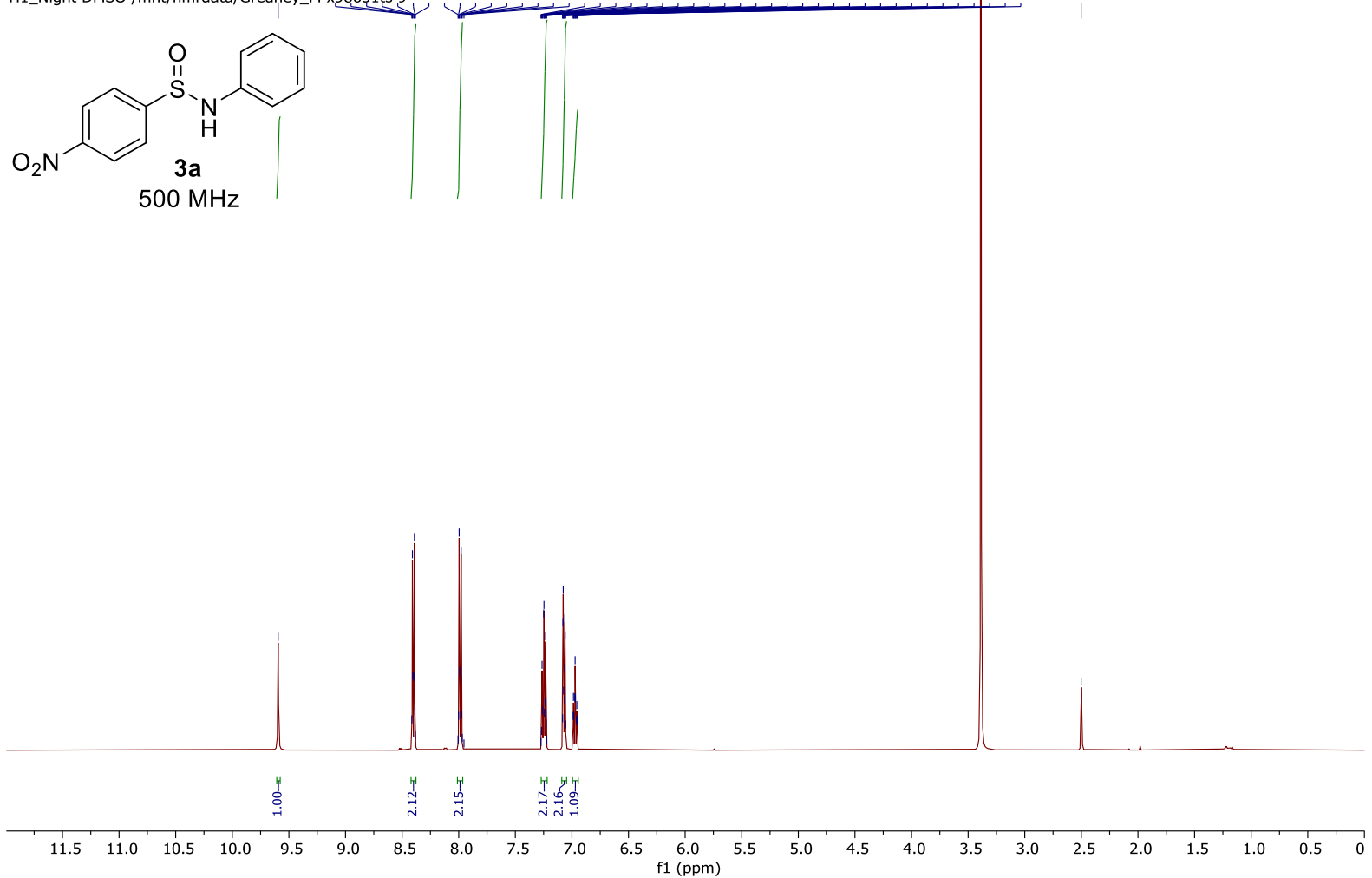
20210329-1732-B500_B.14-9.11.fid

Ref TS2-15

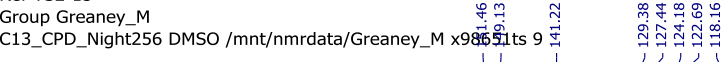<smiles>O=[N+]([O-])c1ccc(S(=O)Nc2ccccc2)cc1</smiles>

$126 \mathrm{MHz}$

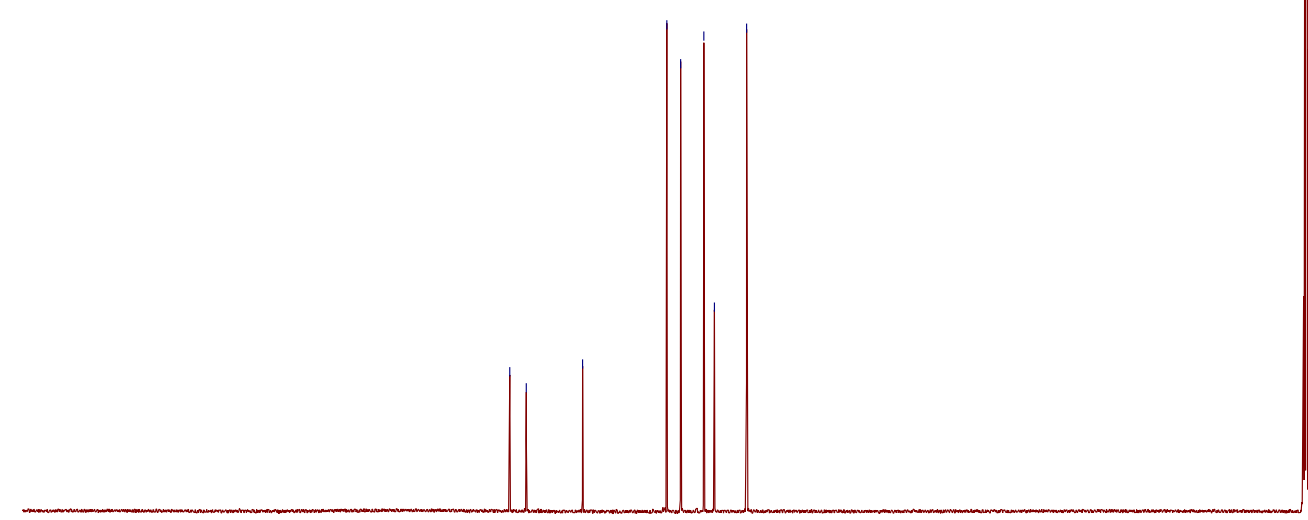

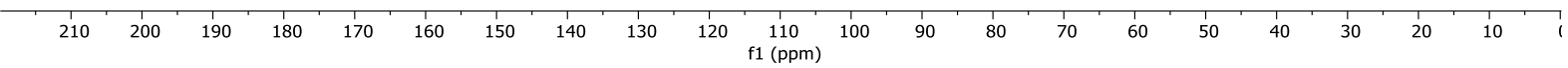

20210924-1445-B500_B.14-45.12.fid

Ref TS2-190FC

Group Greaney M<smiles>Cc1ccccc1NS(=O)c1ccc([N+](=O)[O-])cc1</smiles>

$500 \mathrm{MHz}$

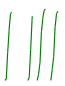

\section{1}

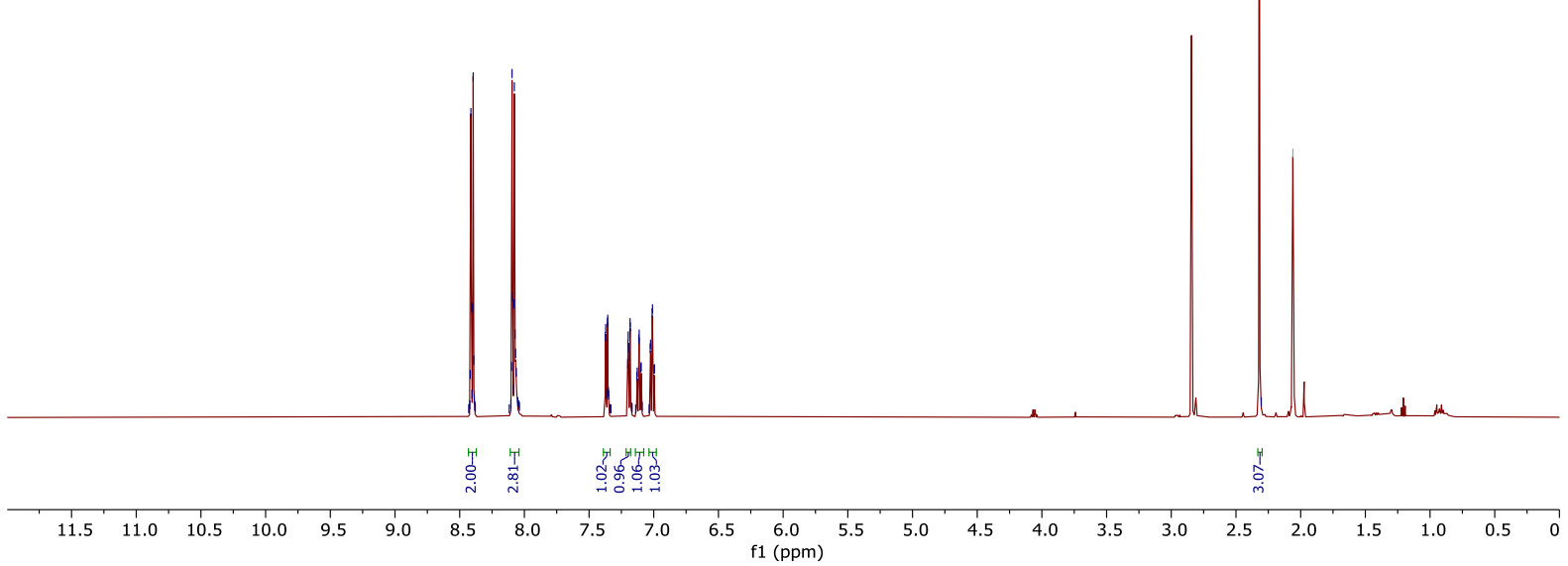


20210924-1445-B500_B.14-45.13.fid

Ref TS2-190FC

Group Greaney_M
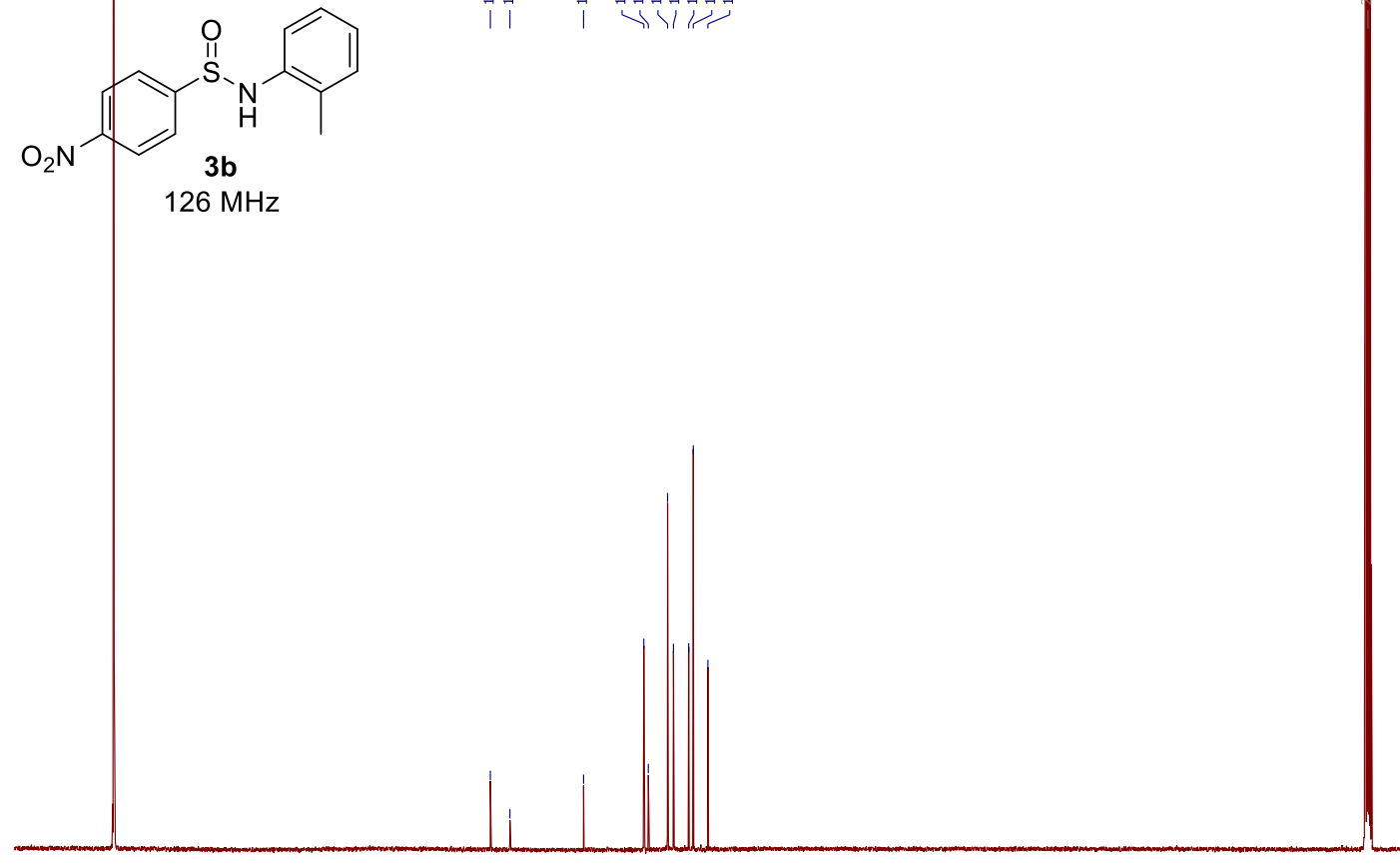

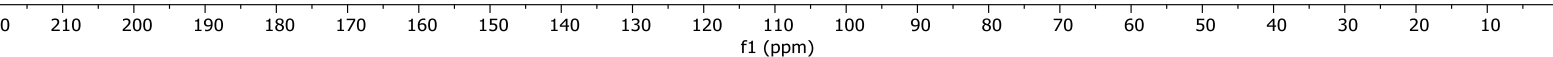

20210524-1144-B500_B.14-31.10.fid Ref TS2-68

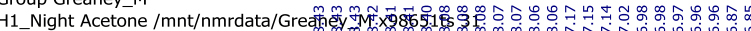<smiles>Cc1cccc(NS(=O)c2ccc([N+](=O)[O-])cc2)c1</smiles>

$500 \mathrm{MHz}$

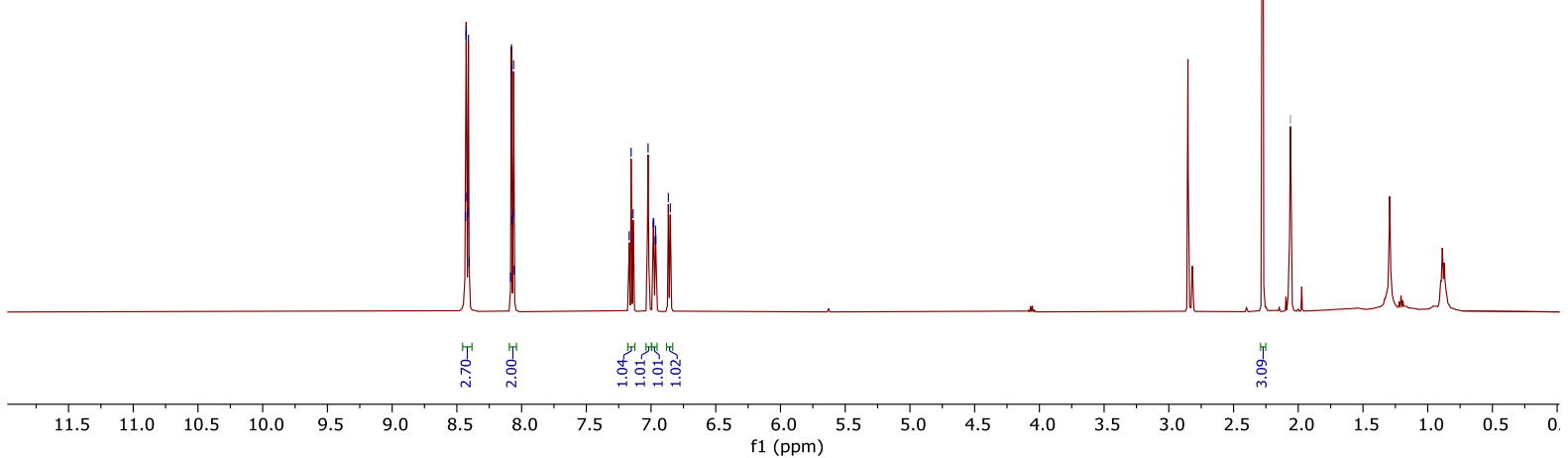


20210524-|144-B500_B.14-31.11.fid

Ref TS2-68

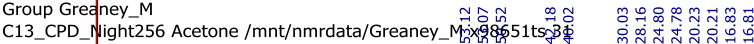

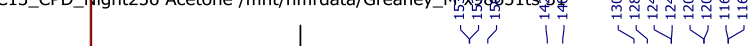

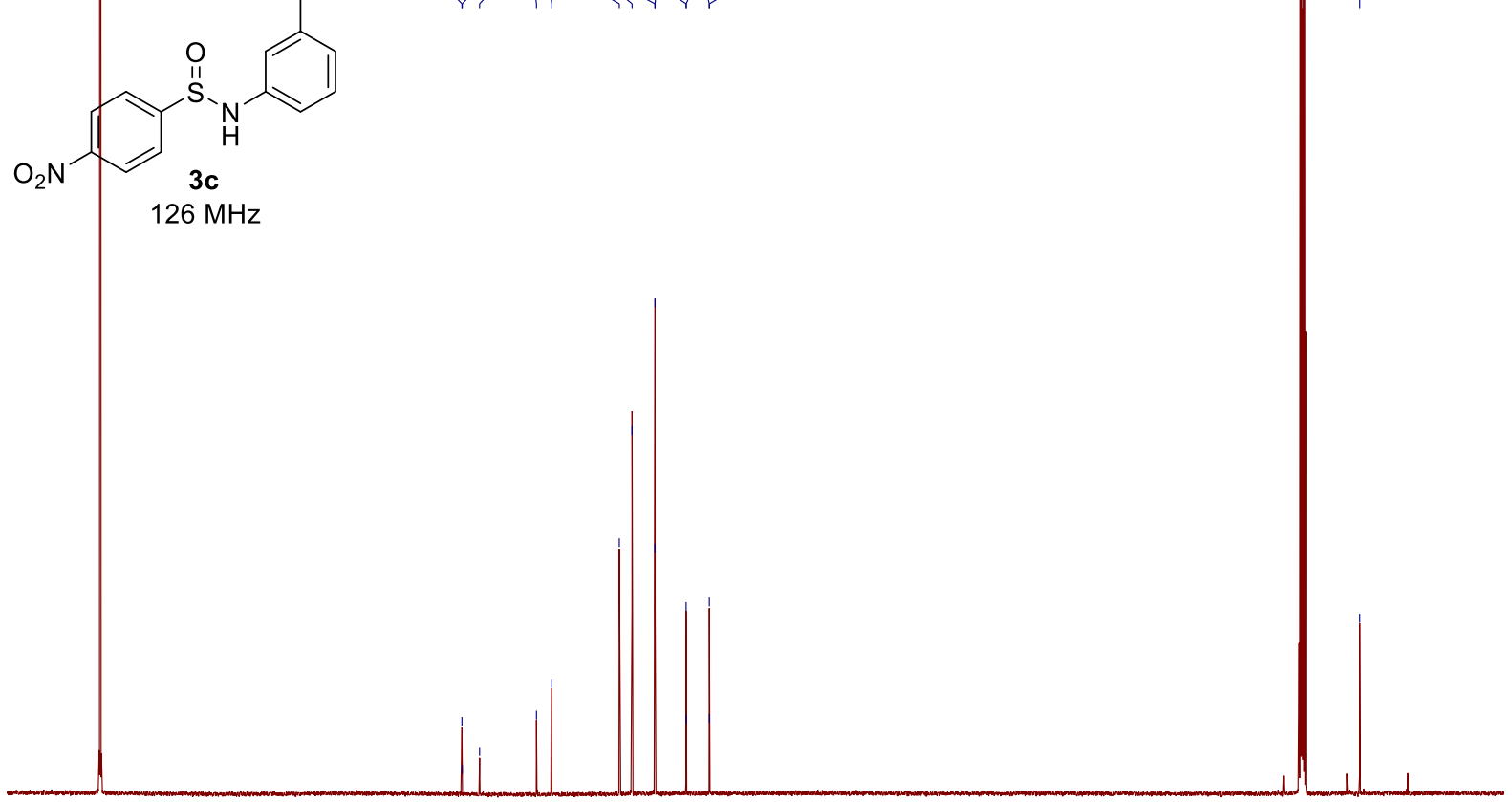

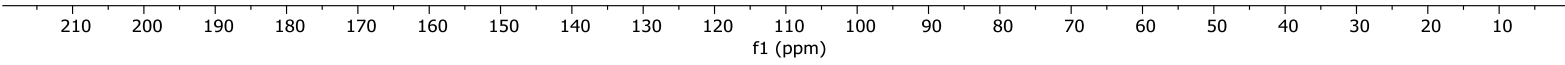

20210513-1610-B400_B.11-21.10.fid

Ref TS2-57

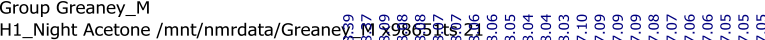
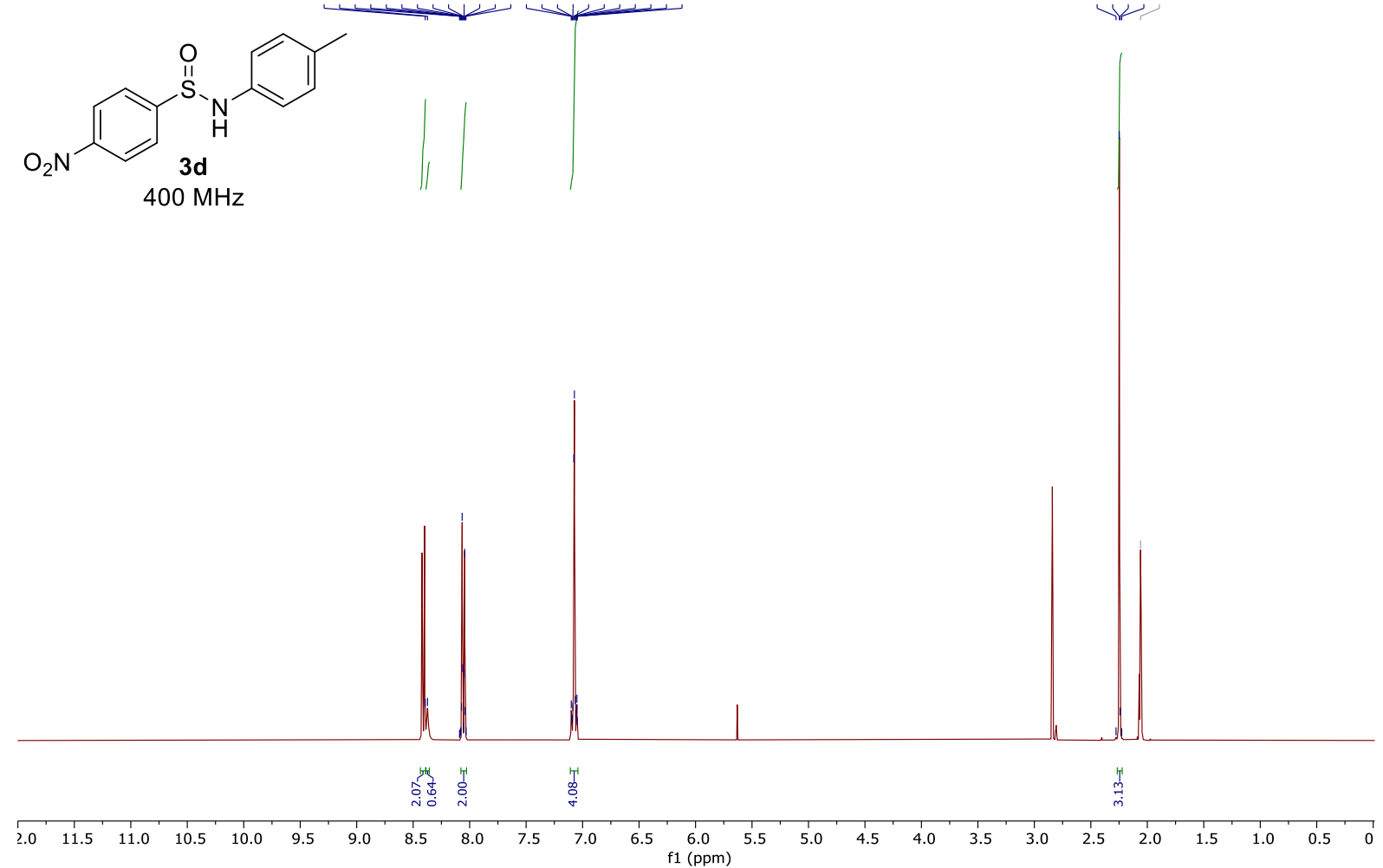
20210513-1610-B400_B.11-21.11.fid

Ref TS2-57

Group Greaney_M

O<smiles>Cc1ccc(NS(=O)c2ccc([N+](=O)[O-])c(C)c2)cc1</smiles>

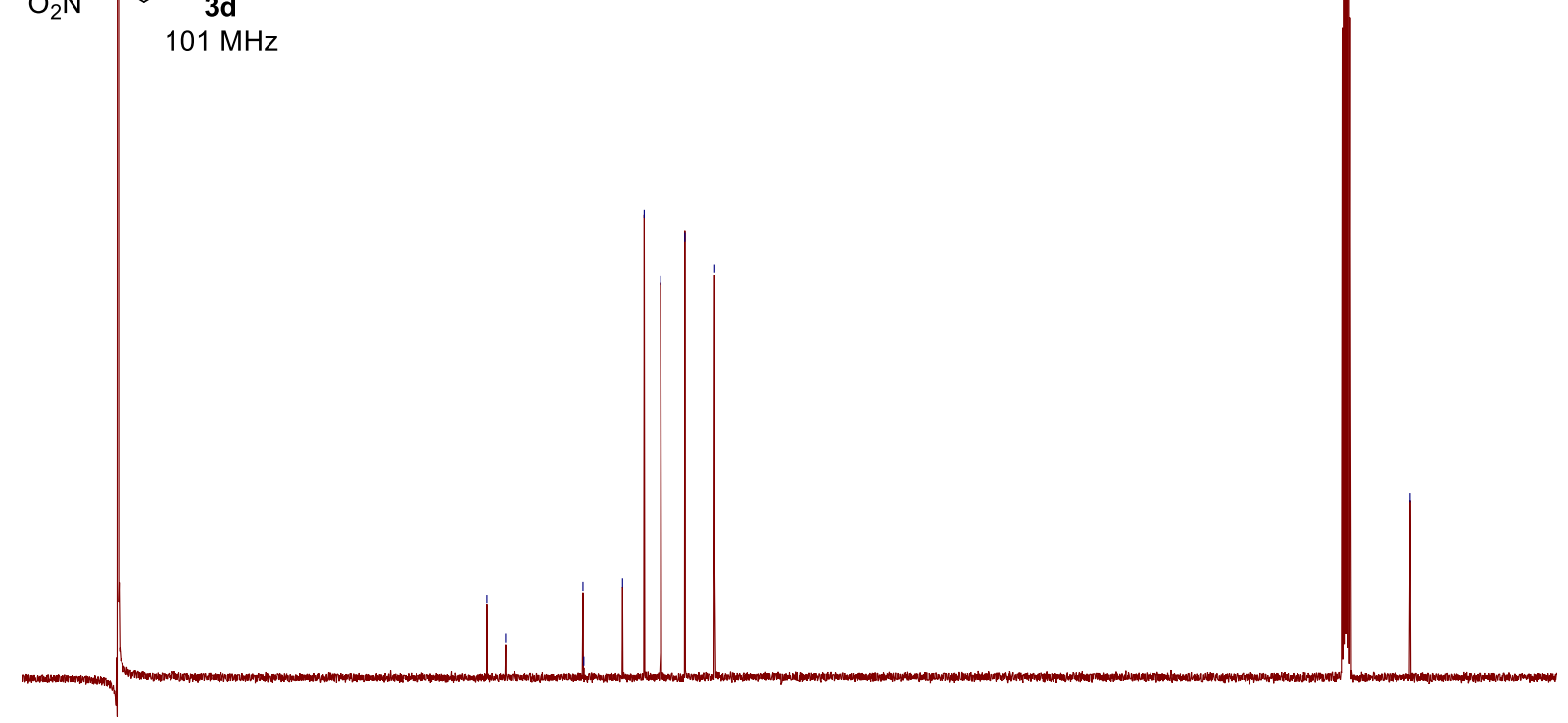

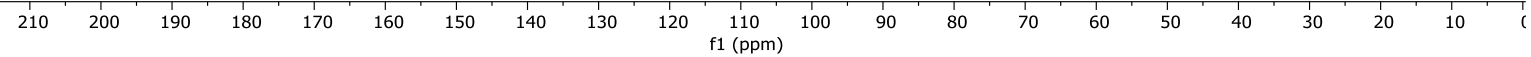

20220104-1515-B500_B.14-8.10.fid

Ref TS3-99a

H1_Day Acetone /mnt/nmrdata/Greaney_M x988ิm<smiles>C#CCCCC</smiles>

$3 e$

$400 \mathrm{MHz}$

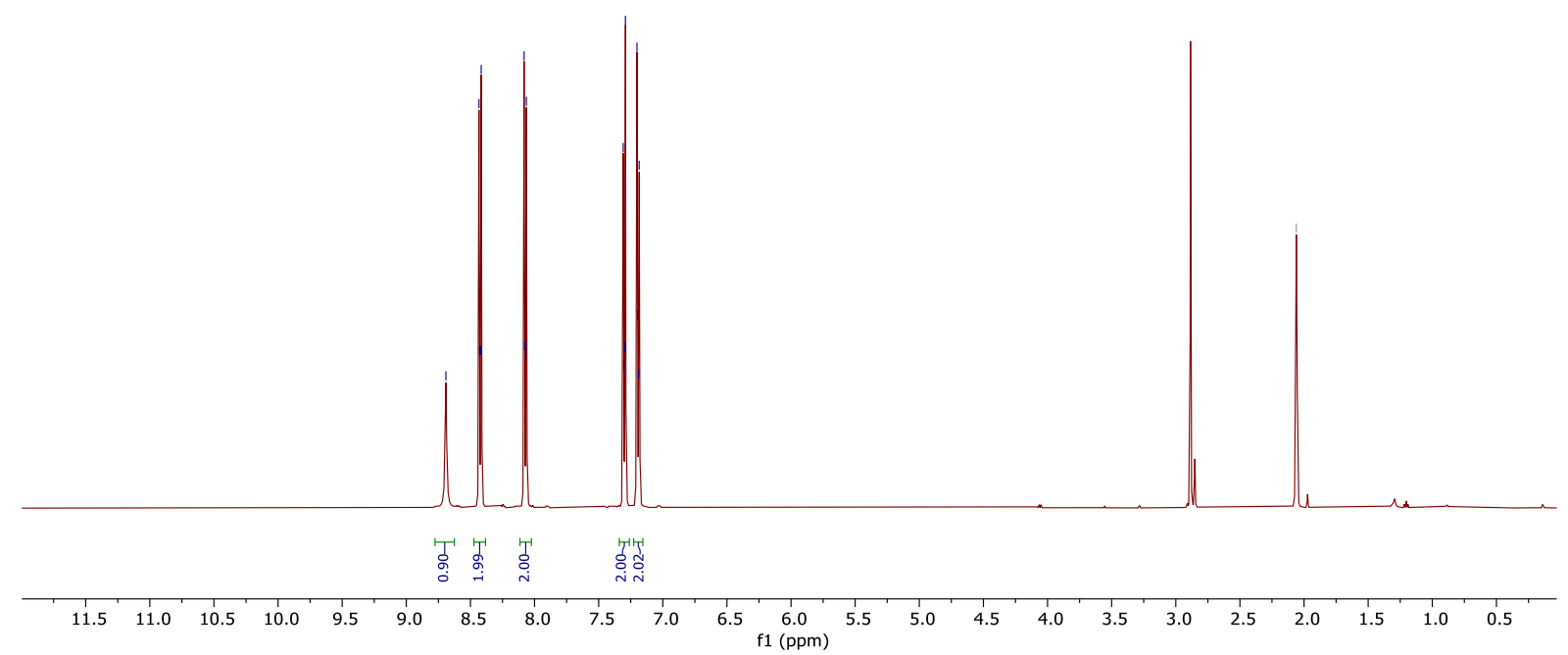


20220104-|515-B500_B.14-8.11.fid

Ref TS3-99a

Group Greaney_M

(I) ।<smiles>Cc1ccc(S(=O)Nc2ccc(Cl)cc2)cc1[N+](=O)[O-]</smiles>

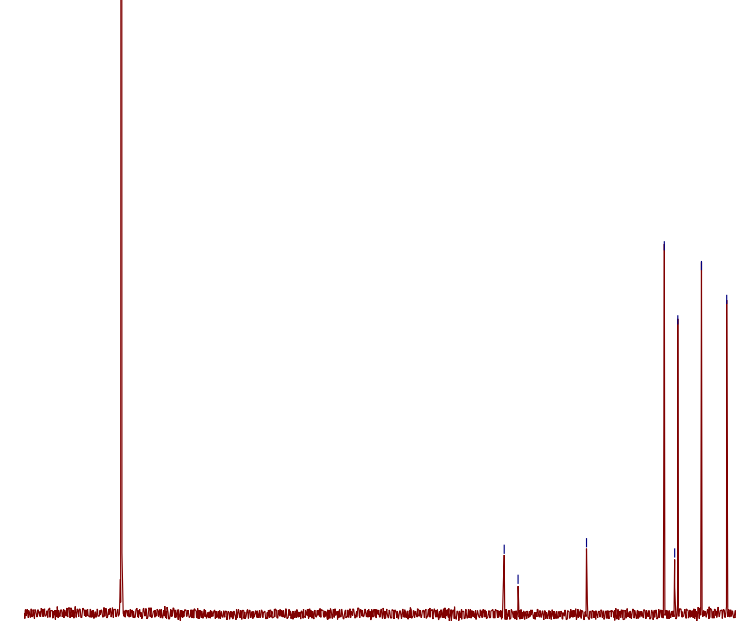

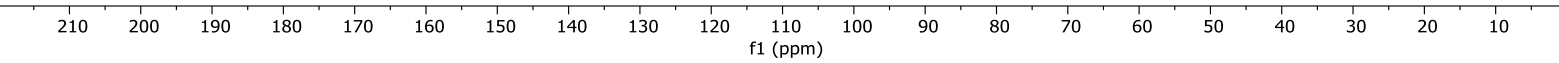

20210312-1738-B400_B.12-29.10.fid

Ref TS1-154 C

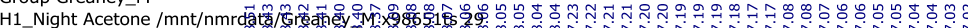<smiles>C[14C](=O)Nc1ccc([N+](=O)[O-])cc1</smiles>

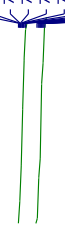

$400 \mathrm{MHz}$

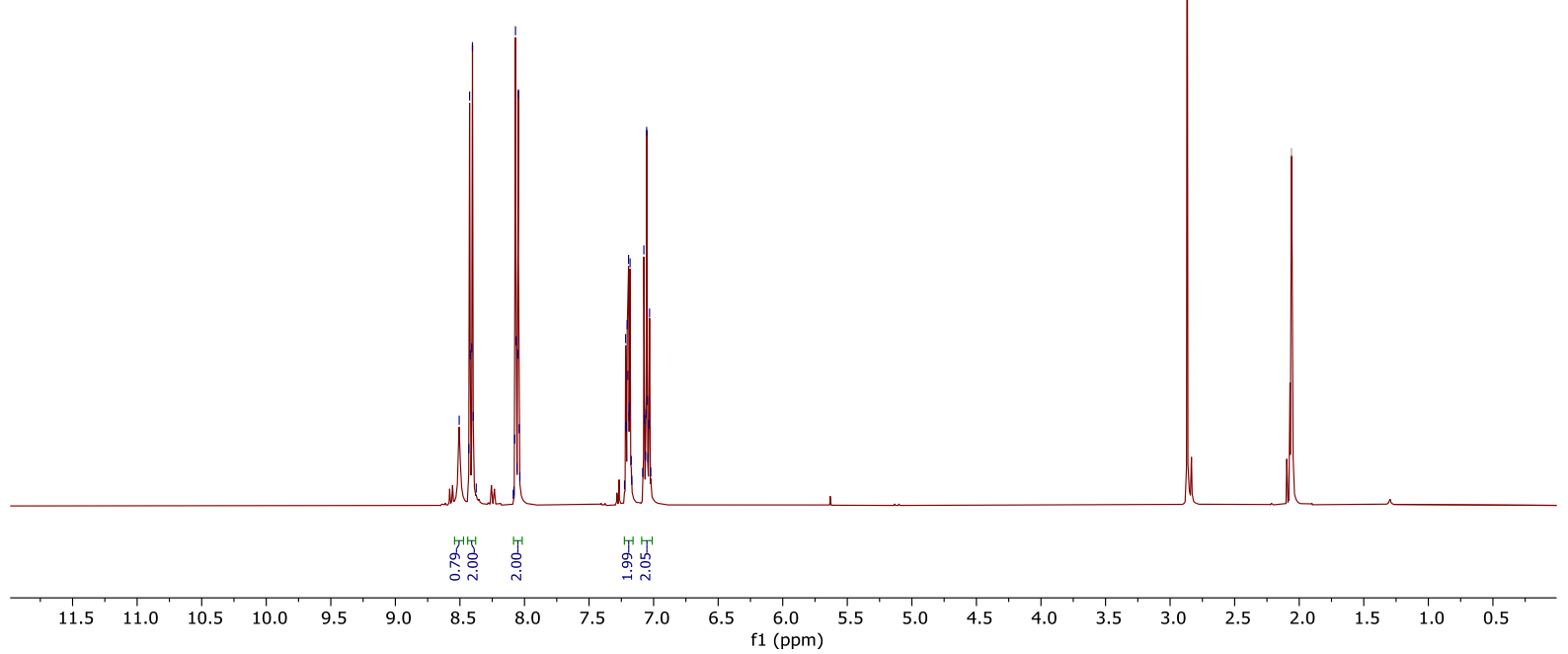


20210312-1738-B400_B.12-29.11.fid

Ref TS1-154 C

Group_Greaney_M

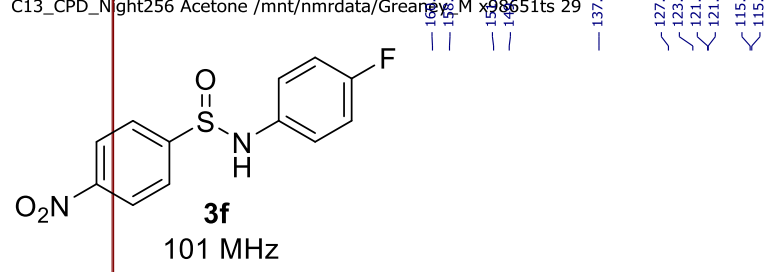

ind

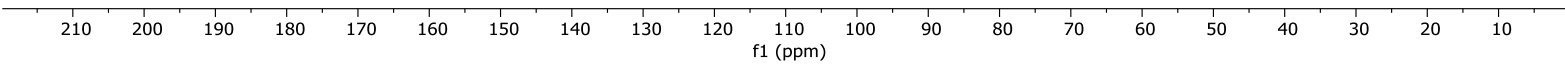

20210312-1738-B400_B.12-29.12.fid

Ref TS1-154 C

F19_CPD_Night Acetone / $\mathrm{mnt} / \mathrm{nmrdata} /$ Greaney_M X98651ts 29<smiles>O=[N+]([O-])c1ccc(S(=O)Nc2ccc(F)cc2)cc1</smiles>

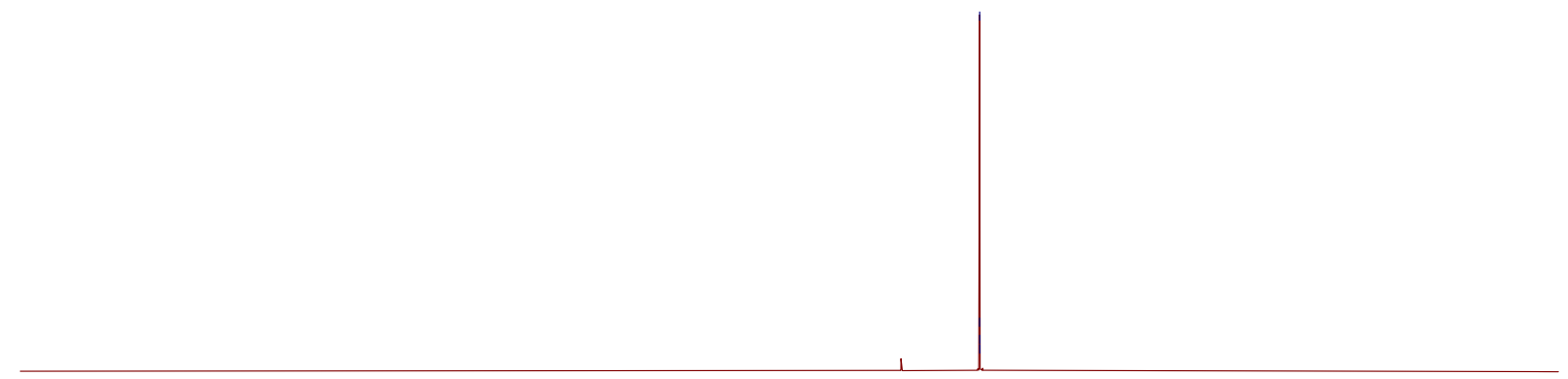

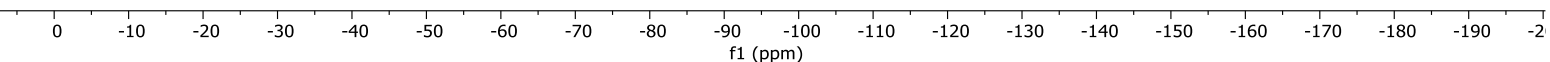


20210625-0950-B500_B.14-41.10.fid

Ref TS2-75C2

Group Greaney_M<smiles>O=[N+]([O-])c1ccc(S(=O)Nc2cccc(Br)c2)cc1</smiles><smiles>C1#CC#C1</smiles>

$500 \mathrm{MHz}$

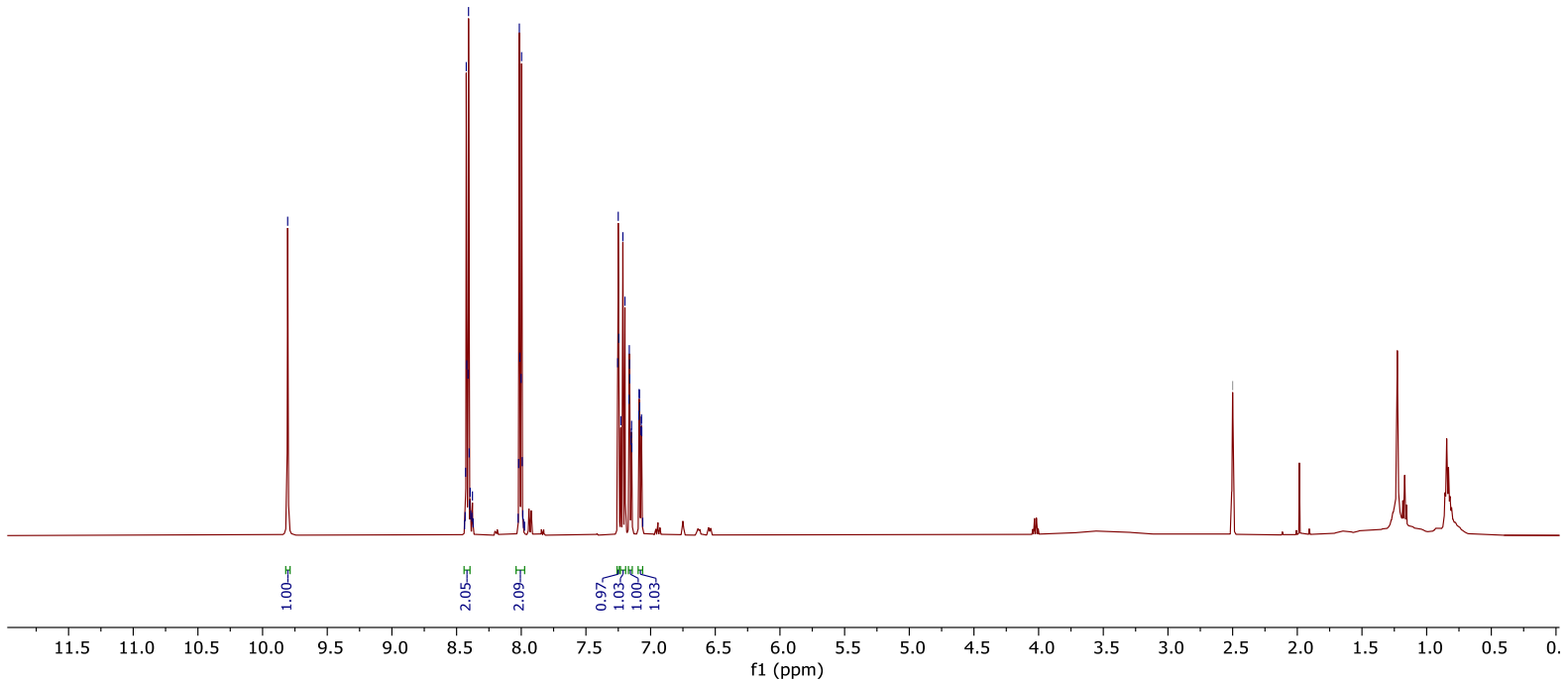

20210625-0950-B500_B.14-41.11.fid

Ref TS2-75C2

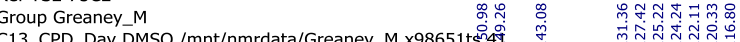<smiles>O=[N+]([O-])c1ccc(S(=O)Nc2cccc(Br)c2)cc1</smiles>

$126 \mathrm{MHz}$

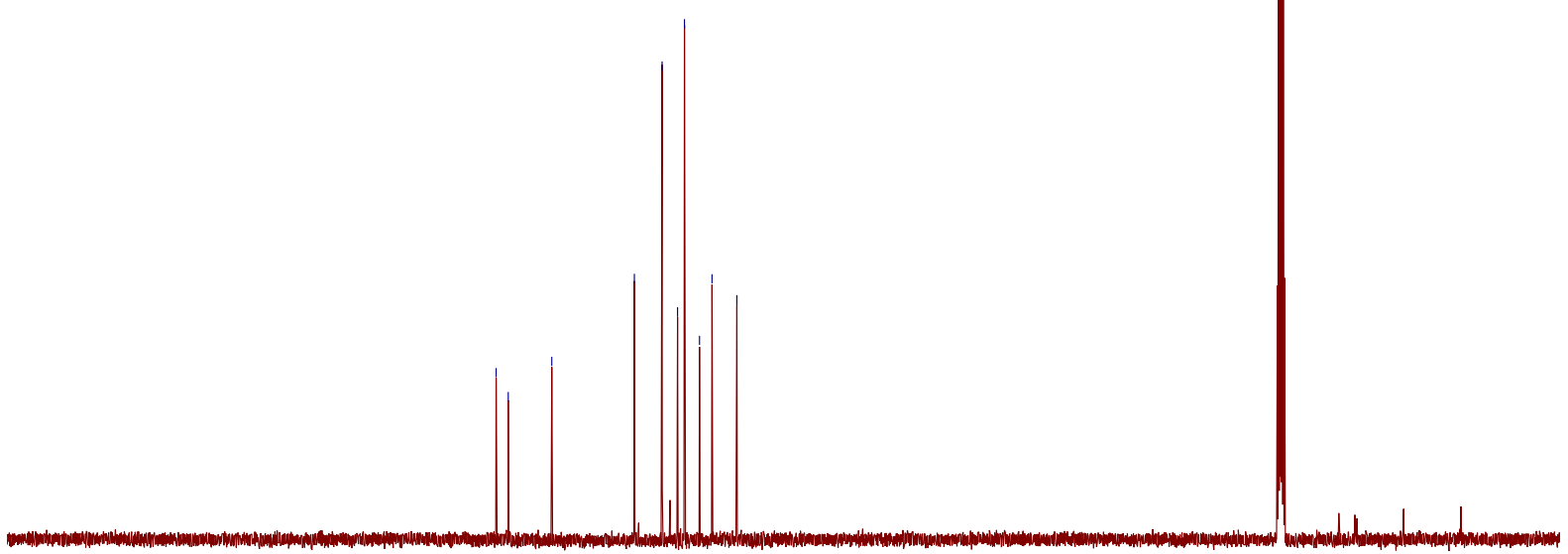

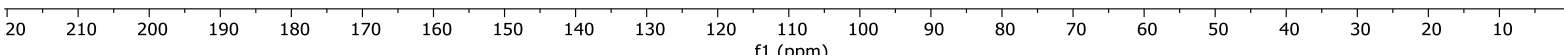


20210625-0950-B500_B.14-42.10.fid

Ref TS2-76C2

Group Greaney_M<smiles>[R14]OS(=O)(=O)c1ccc(S(=O)Nc2ccccc2F)cc1</smiles>

$500 \mathrm{MHz}$

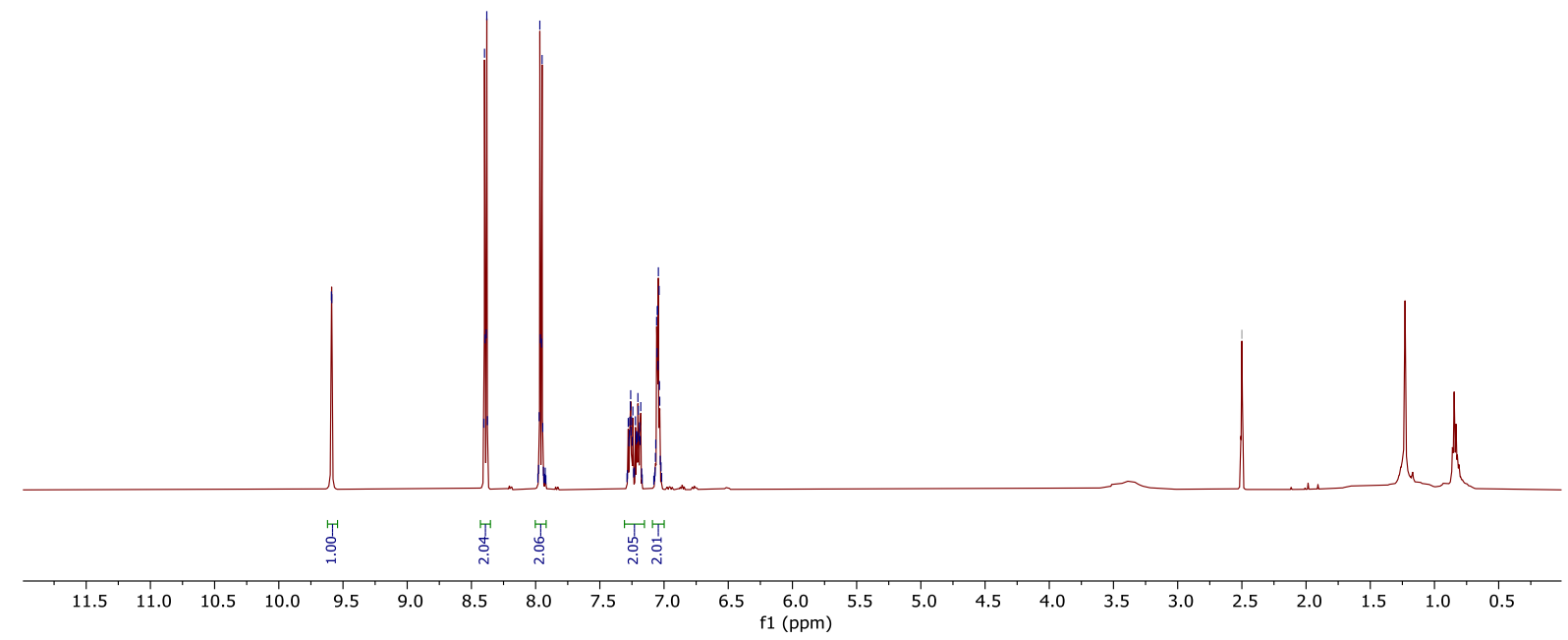

20210625-0950-B500_B.14-42.11.fid Ref TS2-76C2

Group Greaney_M<smiles>O=[N+]([O-])c1ccc(S(=O)Nc2ccccc2F)cc1</smiles>

$126 \mathrm{MHz}$

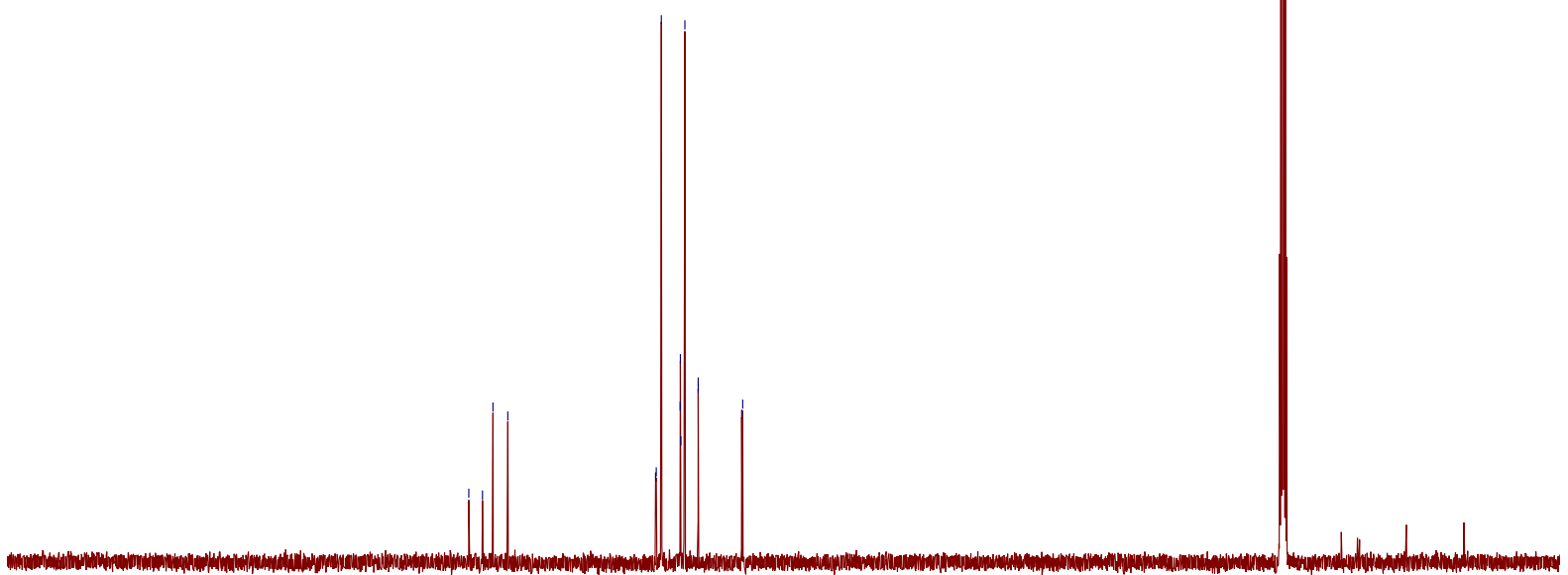

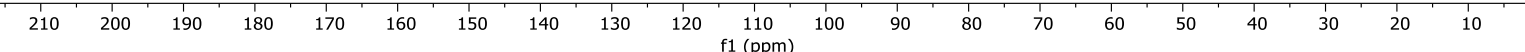


20210625-0950-B500_B.14-42.12.fid

Ref TS2-76C2

Group Greaney_M

F19_NoCPD_Day DMSO /mnt/nmrdata/Greaney_M x98651ts 42<smiles>O=[N+]([O-])c1ccc(S(=O)Nc2ccccc2F)cc1</smiles>

$3 \mathrm{~h}$

$376 \mathrm{MHz}$

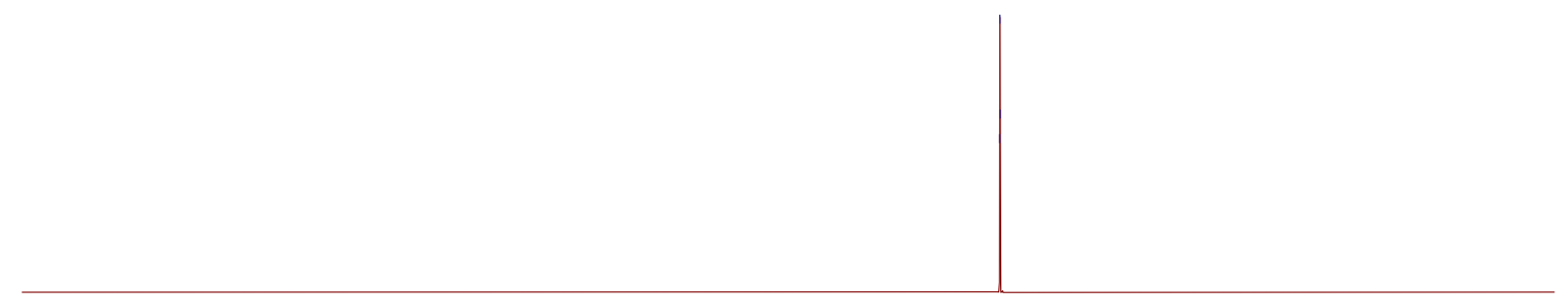

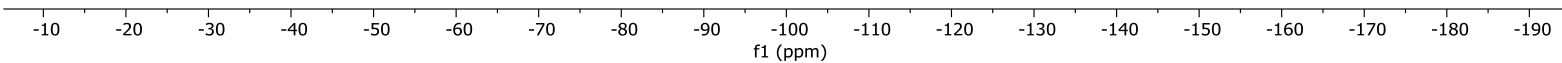

20210510-1614-B400_B.11-42.10.fid Ref TS2-51C

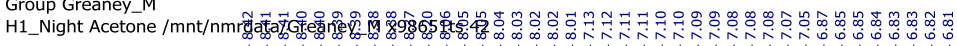<smiles>COc1ccc(NS(=O)c2ccc([N+](=O)[O-])cc2)cc1</smiles>

$400 \mathrm{MHz}$

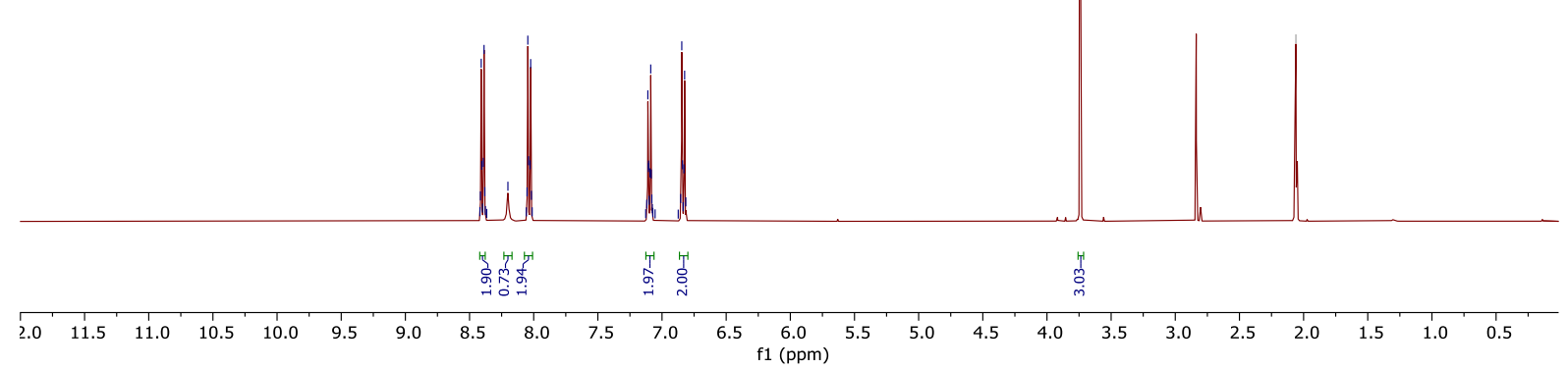


20210510-1614-B400_B.11-42.11.fid

Ref TS2-51C

Group Greaney_M

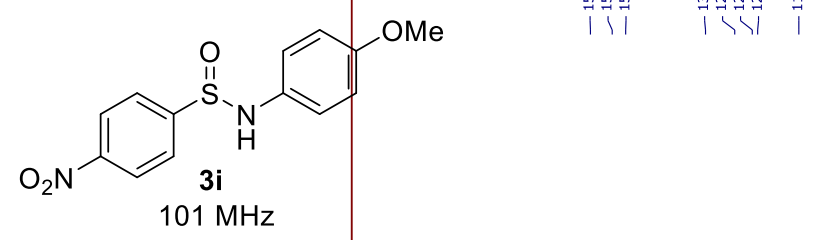

|

$101 \mathrm{MHz}$

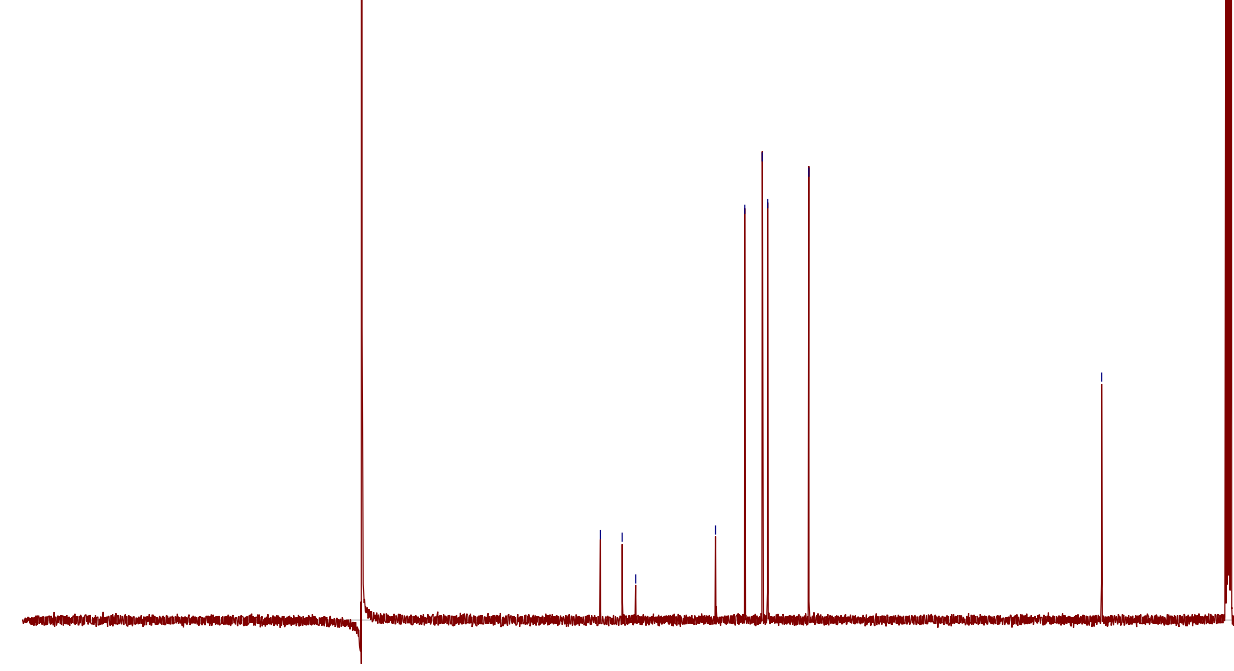

\begin{tabular}{lllllllllllllllllllllllllllllllllllllllllllllllllll}
\hline 270 & 260 & 250 & 240 & 230 & 220 & 210 & 200 & 190 & 180 & 170 & 160 & 150 & 140 & 130 & 120 & 110 & 100 & 90 & 80 & 70 & 60 & 50 & 40 & 30 & 20 & 10 & 0 & -10 & -20 & -30 & -40
\end{tabular}

20210809-1553-B400_B.11-12.10.fid

Ref TS2-136FC

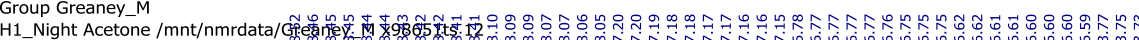

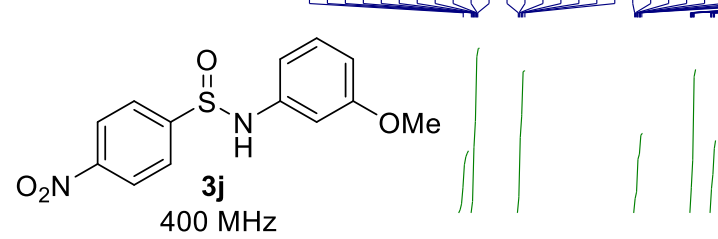

$400 \mathrm{MHz}$

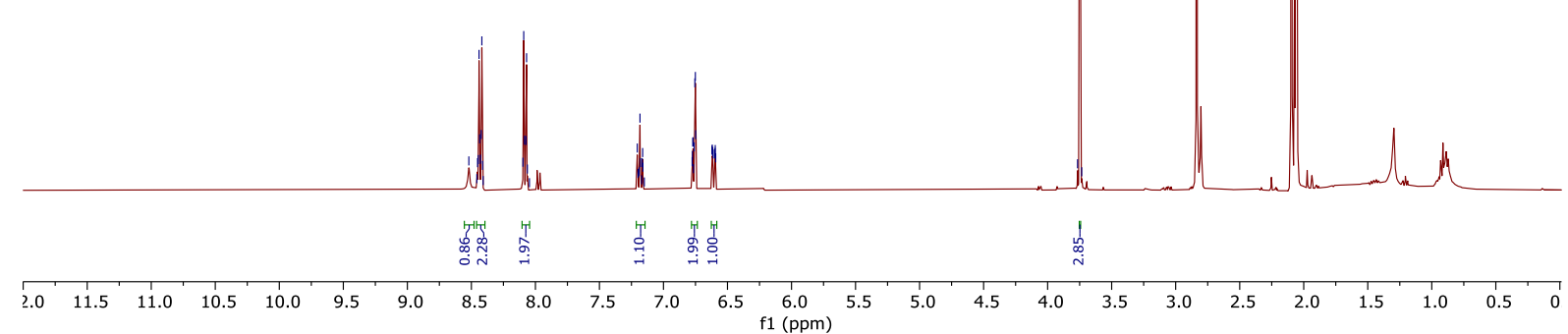


20210809-1553-B400_B.11-12.11.fid

Ref TS2-136FC

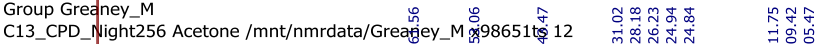

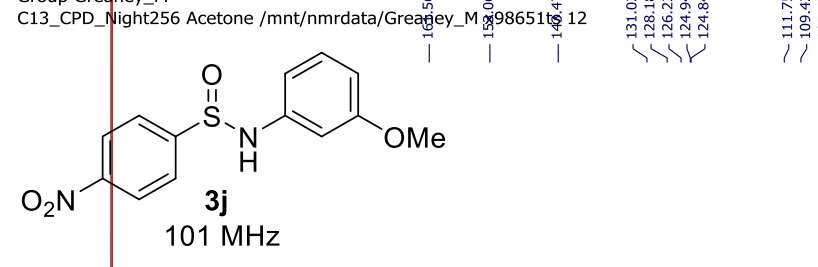

11

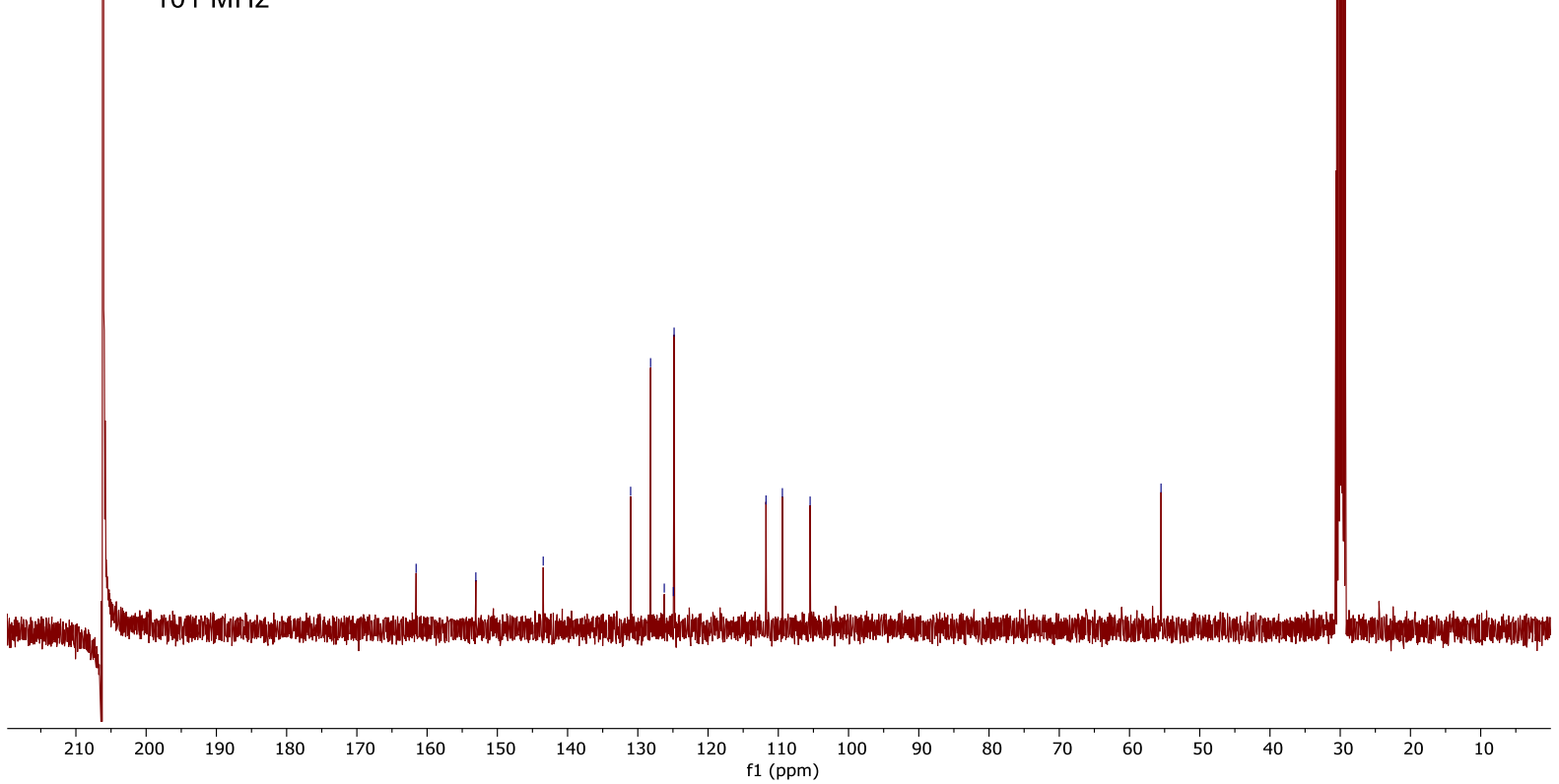

20210818-1446-B400_B.11-50.10.fid

Ref TS2-135FC

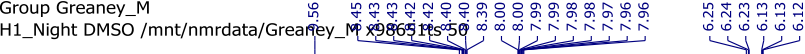<smiles>COc1cc(NS(=O)c2ccc([N+](=O)[O-])cc2)cc(OC)c1</smiles>

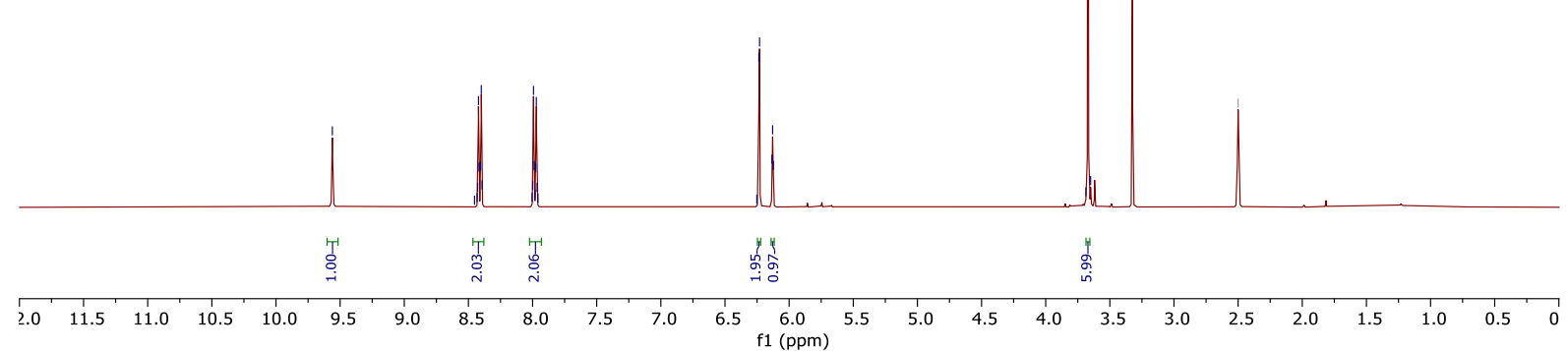


20210818-1446-B400_B.11-50.11.fid

Ref TS2-135FC

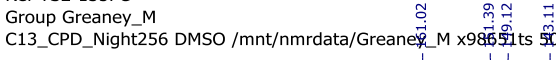

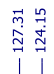

ते
过
11

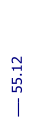<smiles>COc1cc(NS(=O)c2ccc([N+](=O)[O-])cc2)cc(OC)c1</smiles>

$101 \mathrm{MHz}$

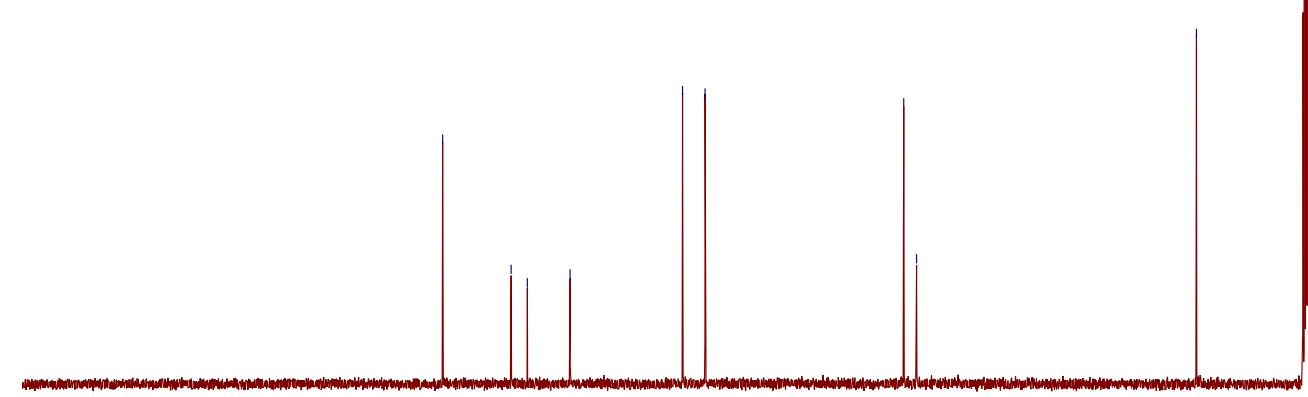

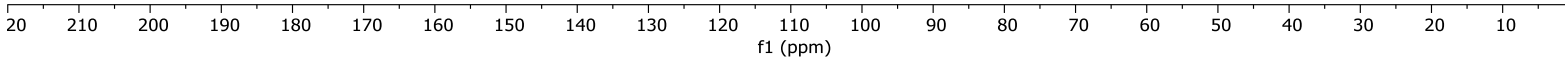

20210520-1809-B400_B.11-11.10.fid

Ref TS2-67C

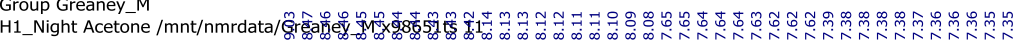<smiles>O=[N+]([O-])c1ccc(S(=O)Nc2ccc(C(F)(F)F)cc2)cc1</smiles>

$3 \mathbf{I}$

$400 \mathrm{MHz}$

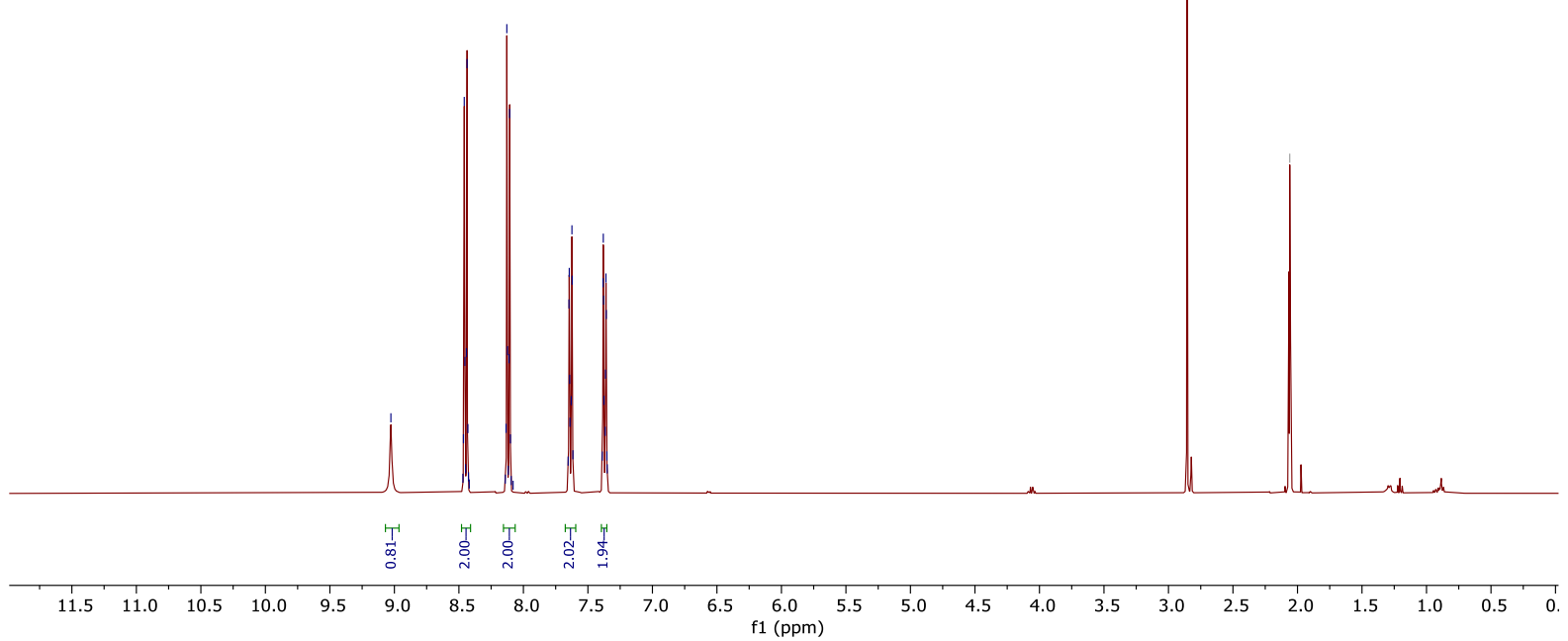


20210520-_809-B400_B.11-11.11.fid

Ref TS2-67)

Group Greaney_M

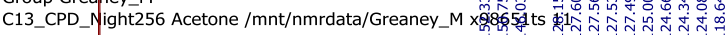

1)<smiles>Cc1ccc(S(=O)Nc2ccc(C(F)(F)F)cc2)cc1[N+](=O)[O-]</smiles>

(n)

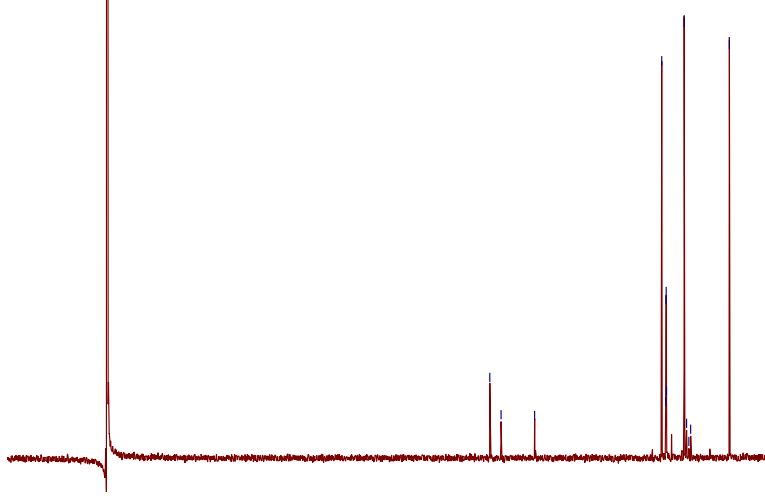

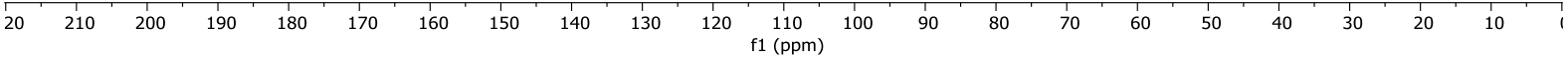

20210520-1809-B400_B.11-11.15.fid

Ref TS2-67C

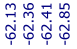

F19_NoCPD_Night Acetone/mnt/nmrdata/Greaney_M x98651ts 11<smiles></smiles>

$376 \mathrm{MHz}$

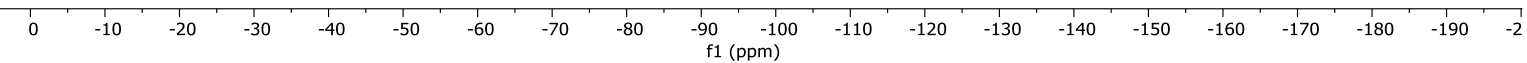


20210625-1422-B500_B.14-6.10.fid

Ref TS2-86C2

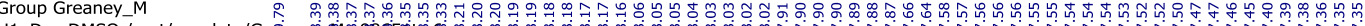

H1_Day DMSO/mnt/nmrdata/Greaney<smiles></smiles>

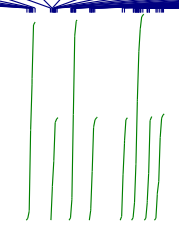

$500 \mathrm{MHz}$

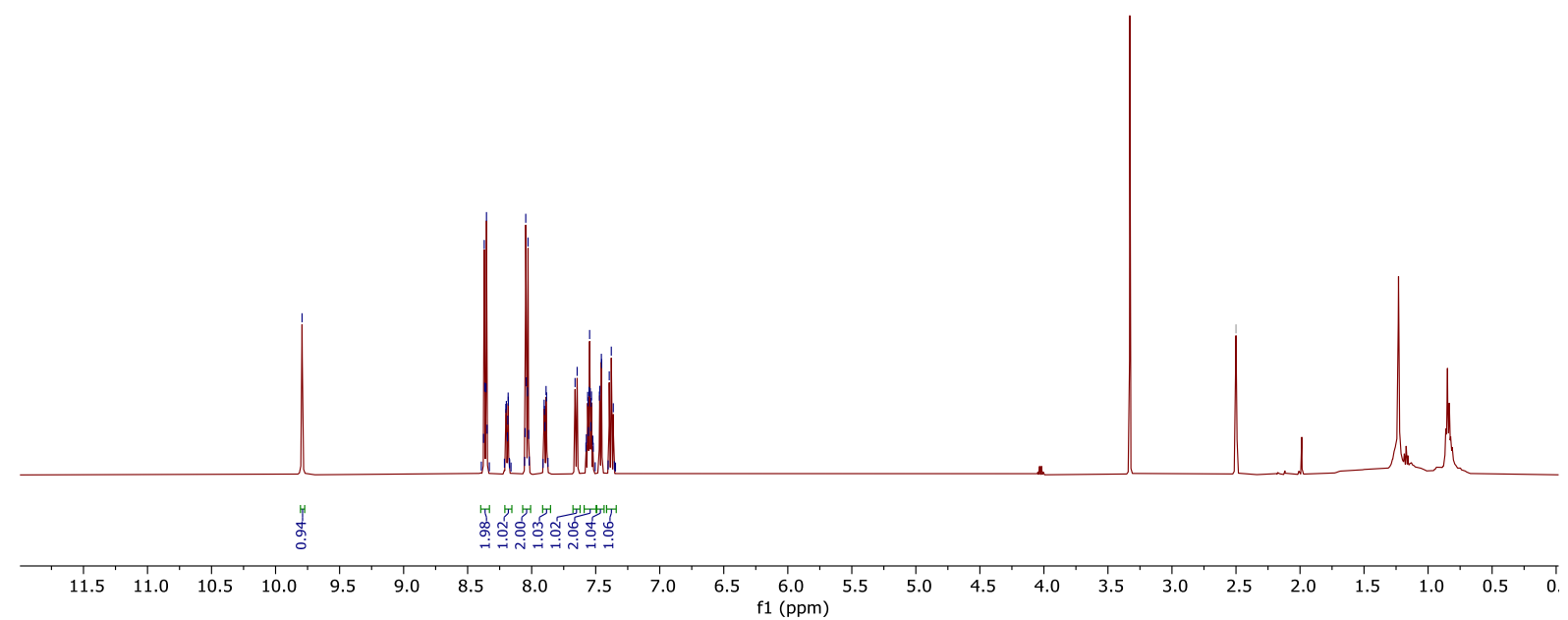

20210625-1422-B500_B.14-6.11.fid

Ref TS2-86C2

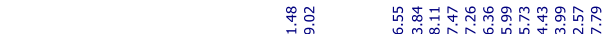

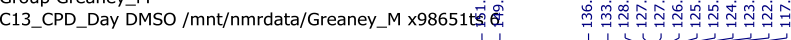<smiles>O=[N+]([O-])c1ccc(S(=O)Nc2cccc3ccccc23)cc1</smiles>

(1)

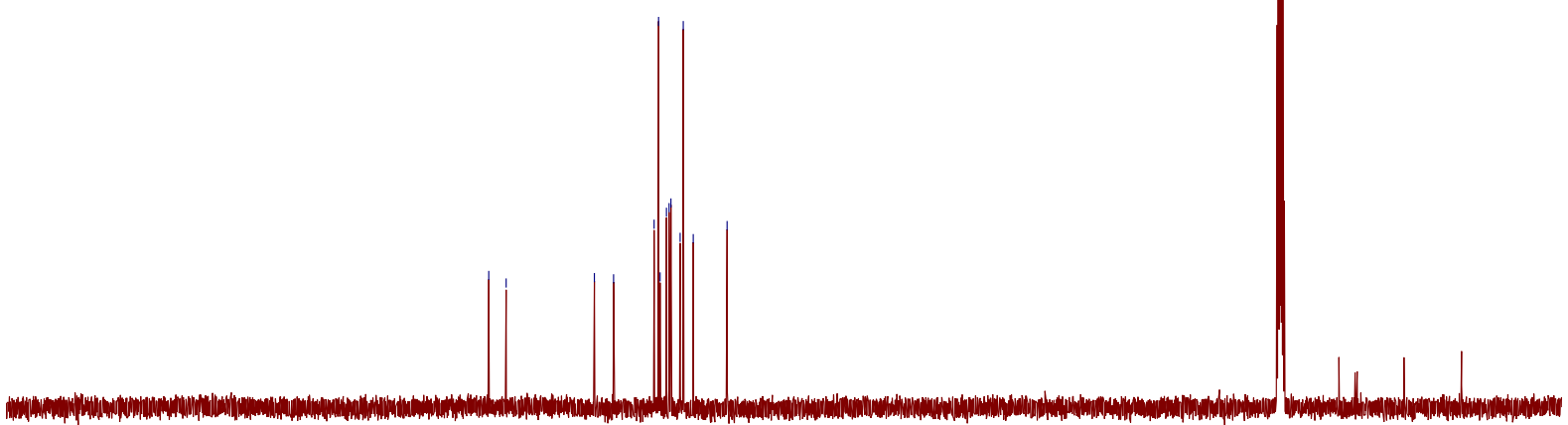

$210 \quad 200 \quad 190 \quad 180 \quad 170 \quad 160$

$140 \quad 130$

$20 \quad 110$ 
20210716-1616-B400_B.11-37.12.fid

Ref TS2-96FC

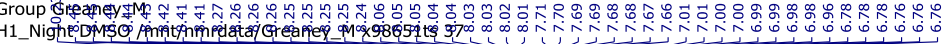
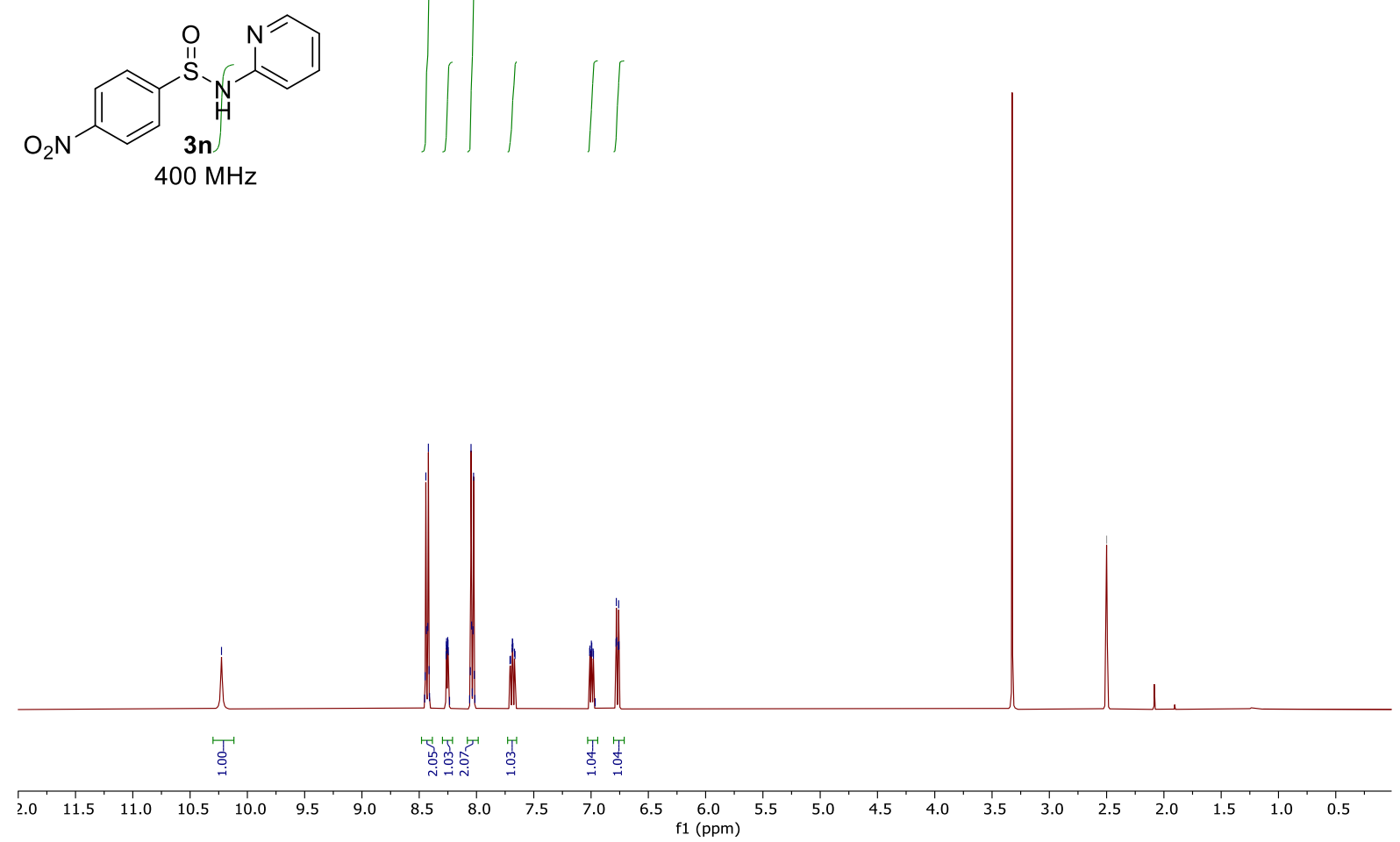

20210716-1616-B400_B.11-37.13.fid

Ref TS2-96FC

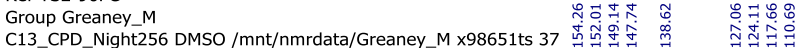<smiles>O=[N+]([O-])c1ccc(S(=O)Nc2ccccn2)cc1</smiles>

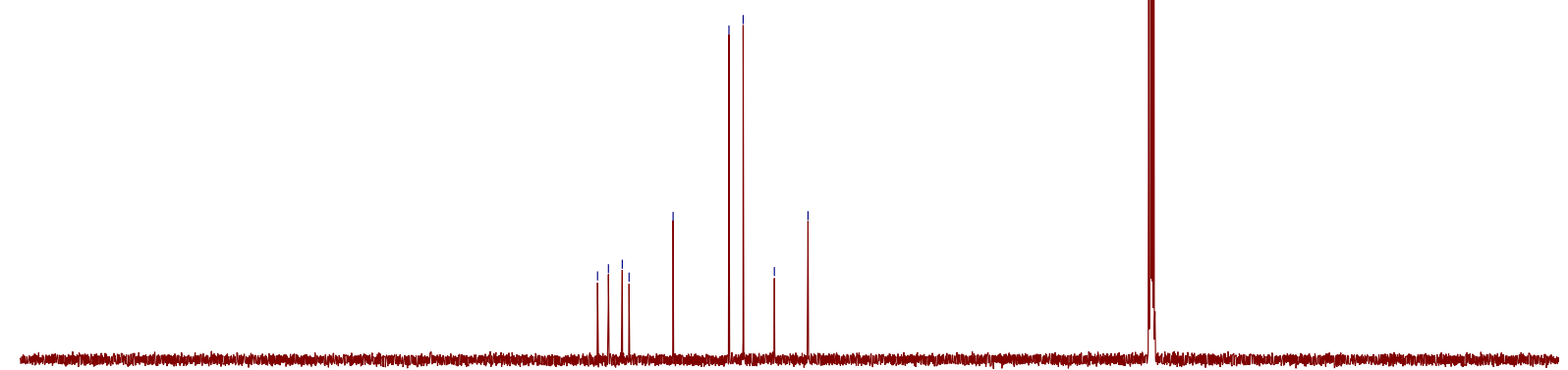

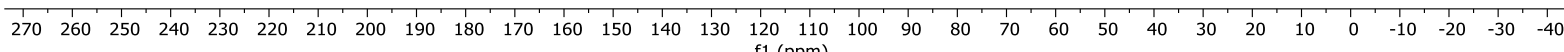
$1(\mathrm{ppm})$ 
20210728-1446-B400_B.11-39.12.fid

Ref TS2-111FC

Group Greaney_M<smiles>O=[N+]([O-])c1ccc(S(=O)Nc2cccc3cccnc23)cc1</smiles>

$400 \mathrm{MHz}$
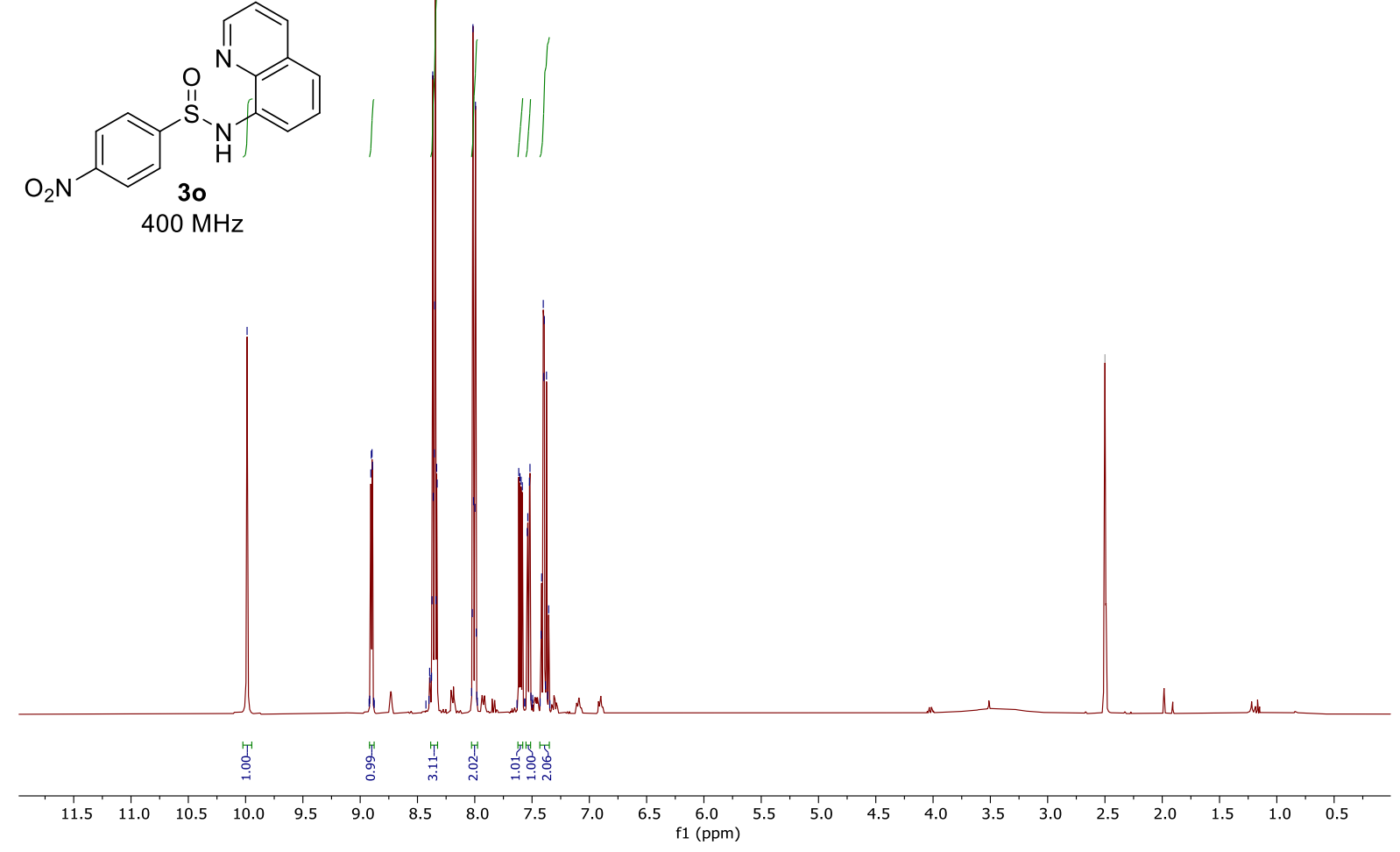

20210728-1446-B400_B.11-39.13.fid

Ref TS2-111FC

(13)<smiles>O=[N+]([O-])c1ccc(S(=O)Nc2cccc3cccnc23)cc1</smiles>

Y l

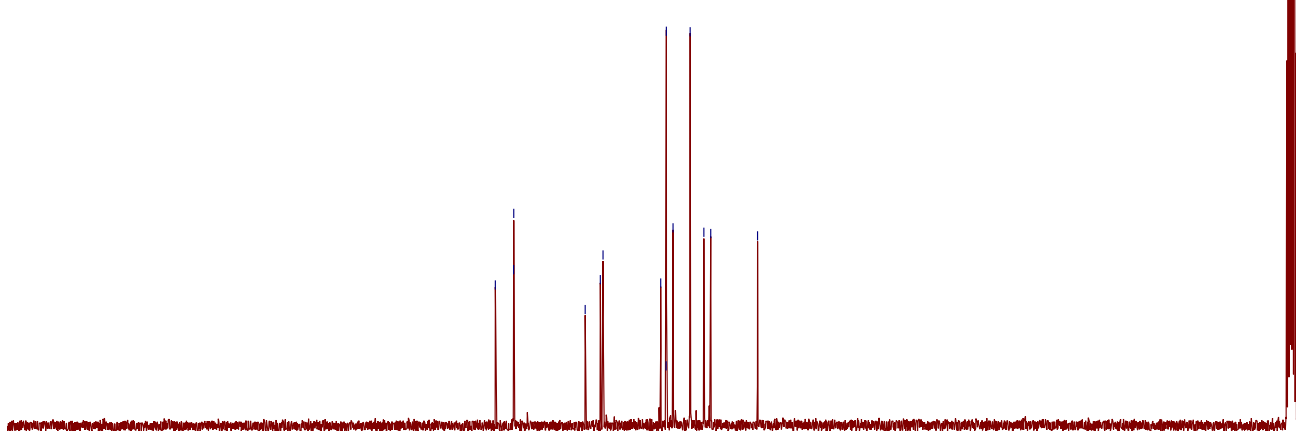

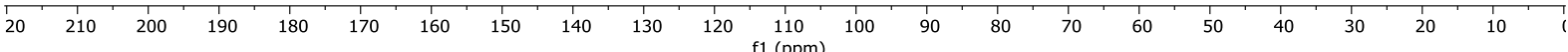


20210625-0951-B500_B.14-43.10.fid

Ref TS2-82C2

Group Greaney $M$

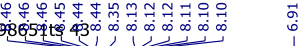

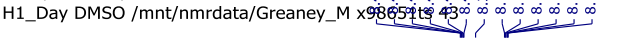<smiles>[R][R6]([R])([R])O[Na]</smiles>

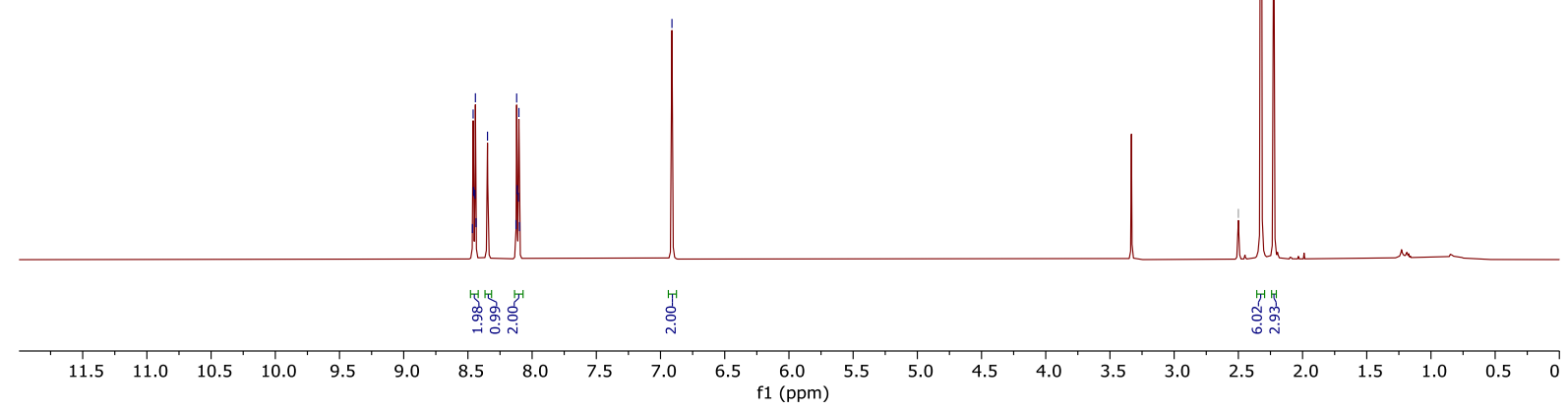

20210625-0951-B500_B.14-43.11.fid

Ref TS2-82C2

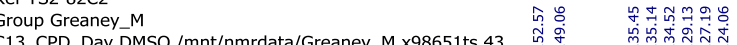

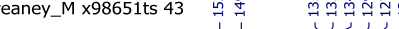<smiles>Cc1cc(C)c(NS(=O)c2ccc([N+](=O)[O-])cc2)c(C)c1</smiles>

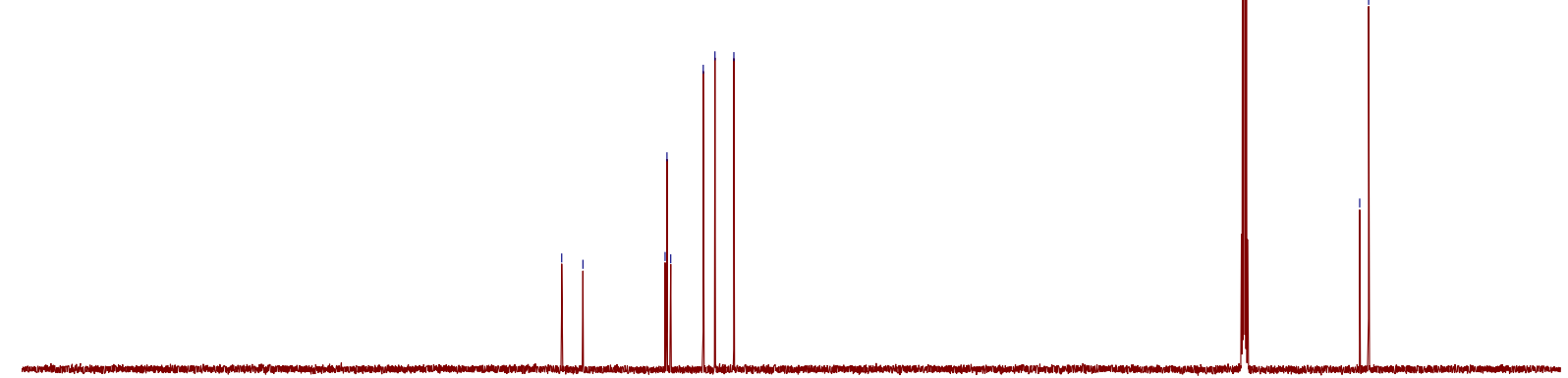

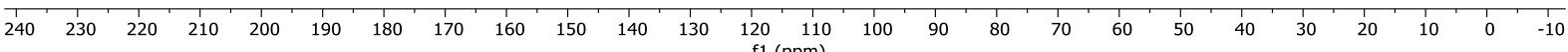


20210802-1702-B400_B.11-49.12.fid

Ref TS2-142FC

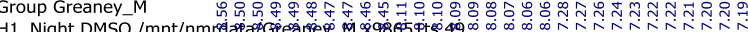

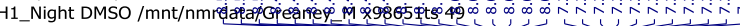<smiles>CCCc1cccc(C(F)(F)F)c1NS(=O)c1ccc([N+](=O)[O-])cc1</smiles>

$400 \mathrm{MHz}$

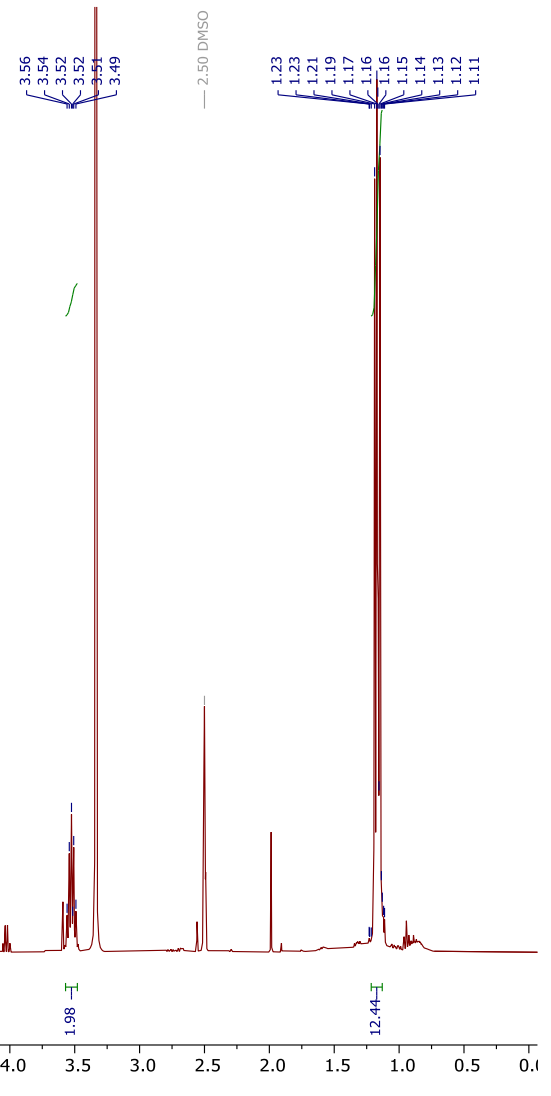

20210802-1702-B400_B.11-49.13.fid

Ref TS2-142FC

C13 CPD Night256 DMSO /mnt/nmrdata/Greaney_M x9810

111<smiles>CC(C)c1cccc(C(C)C)c1NS(=O)c1ccc([N+](=O)[O-])cc1</smiles>

$101 \mathrm{MHz}$

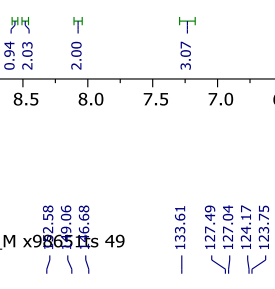

$1(\mathrm{ppm})$ 
20211124-1444-B400_B.11-21.10.fid

Ref TS3-76a

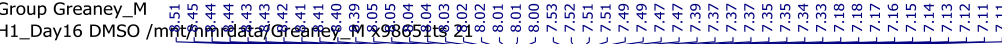<smiles>C[C@H](OC(=O)O)c1ccccc1NS(=O)c1ccc([N+](=O)[O-])cc1</smiles>

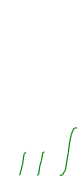

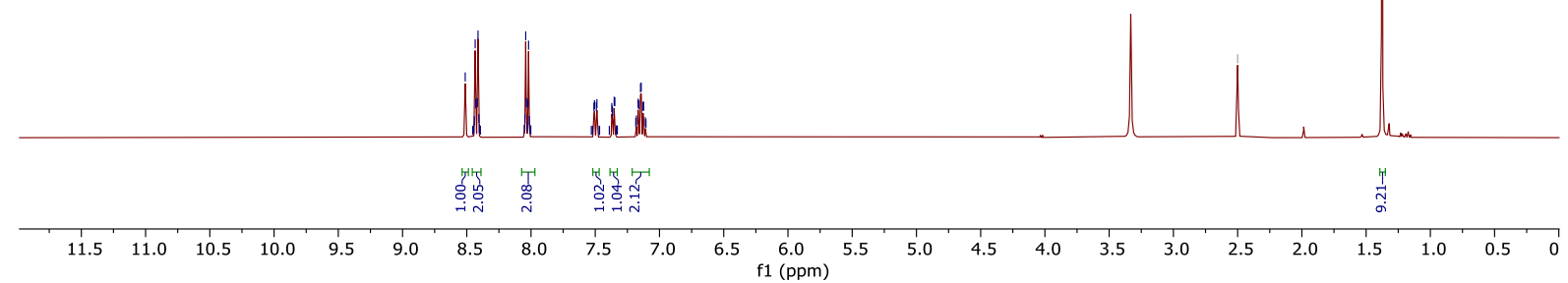

20211124-1444-B400_B.11-21.11.fid

Ref TS3-76a

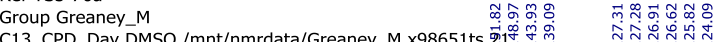

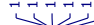<smiles>CC(C)(C)c1ccccc1NS(=O)c1ccc([N+](=O)[O-])cc1</smiles>

101 $\mathrm{MHz}$

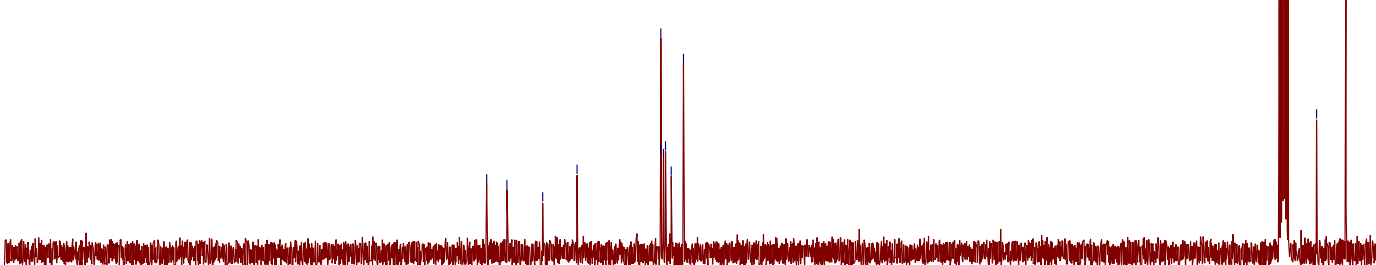

$\begin{array}{lllllllllllllllllllllll}1 & 210 & 200 & 190 & 180 & 170 & 160 & 150 & 140 & 130 & 120 & 110 & 100 & 90 & 80 & 70 & 60 & 50 & 40 & 30 & 20 & 10 & 0\end{array}$ 
20210906-1036-B500_B.14-24.10.fid

Ref TS2-155FC

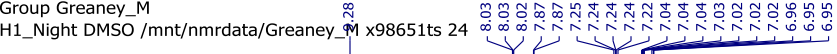
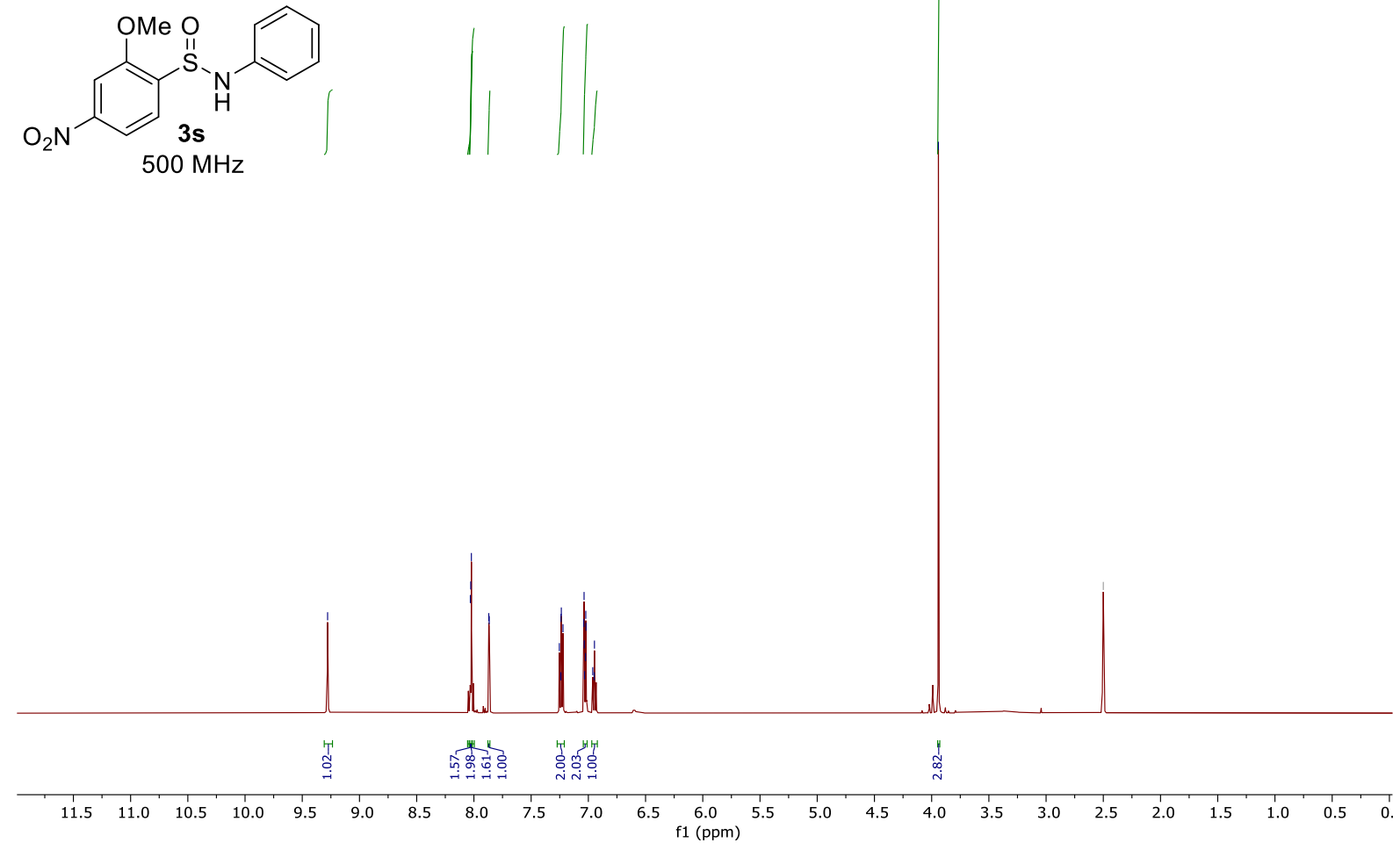

20210906-1036-B500_B.14-24.11.fid

Ref TS2-155FC

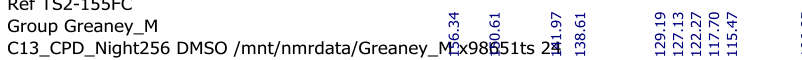

(1)

$126 \mathrm{MHz}$

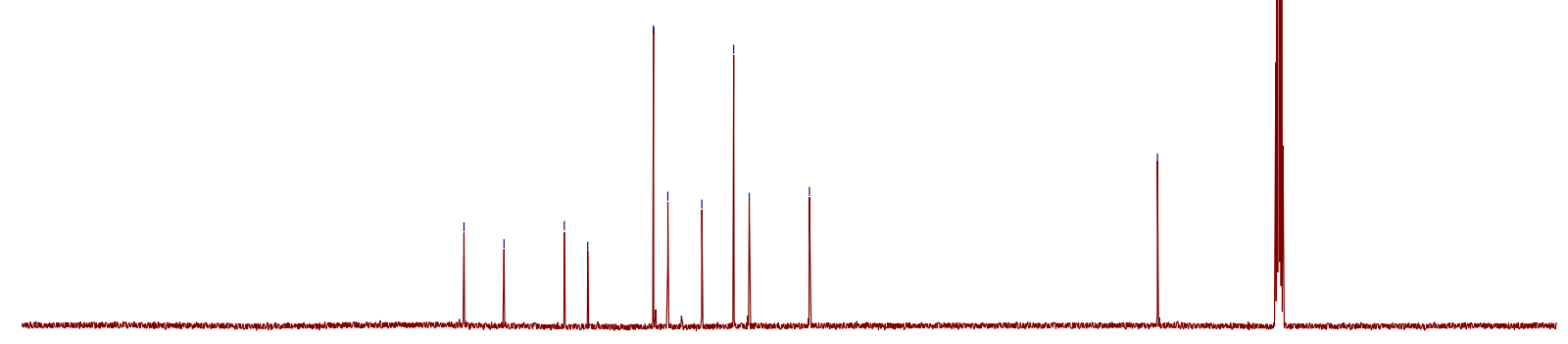

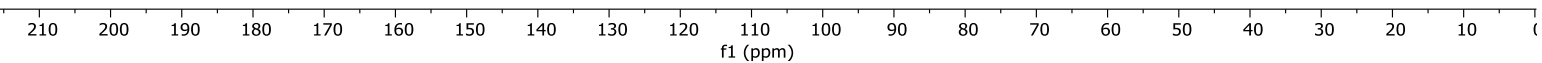


20210910-1215-B500_B.14-8.10.fid

Ref TS2-177FC

Group Greaney_M

1.Night DMSO/mnt/nmd

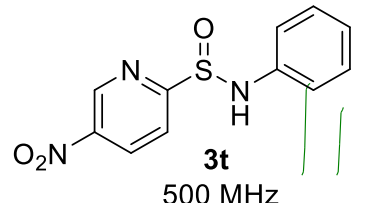

$500 \mathrm{MHz}$

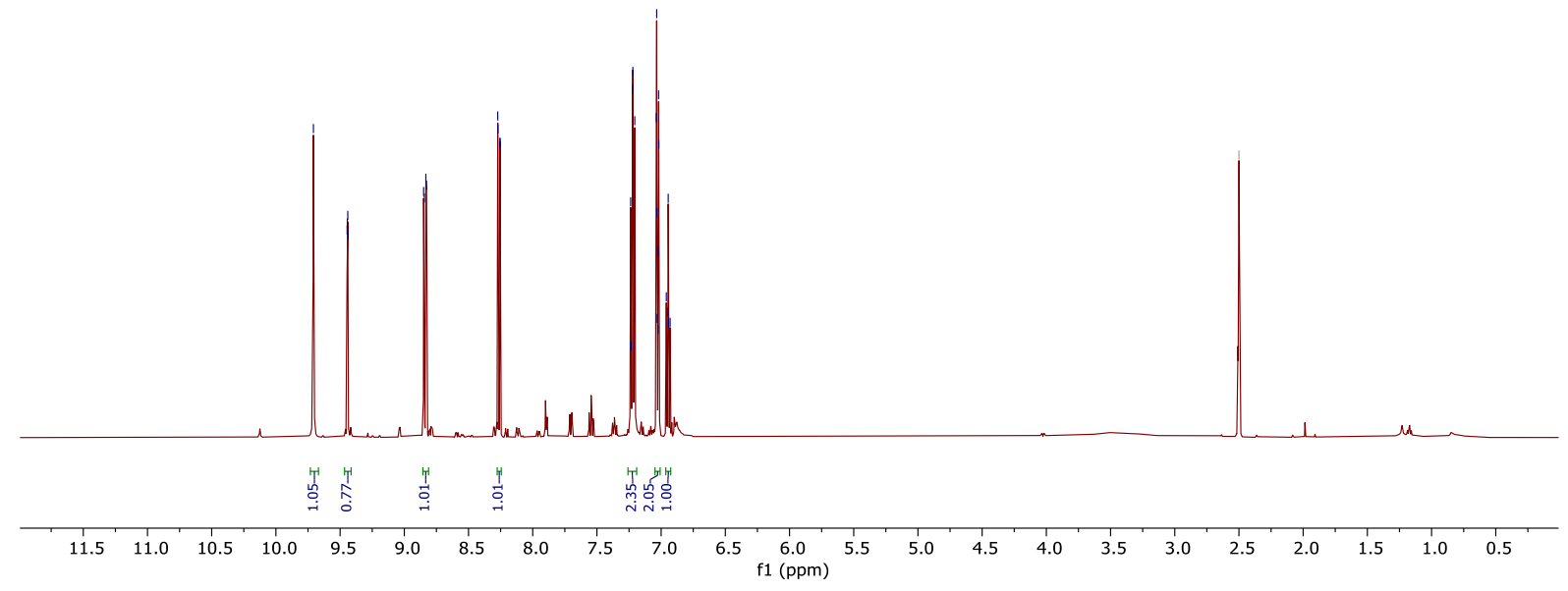

20210910-1215-B500_B.14-8.11.fid

Ref TS2-177FC

D
D.

V | $Y$ |<smiles>O=[N+]([O-])c1ccc(S(=O)Nc2ccccc2)nc1</smiles>

$126 \mathrm{MHz}$

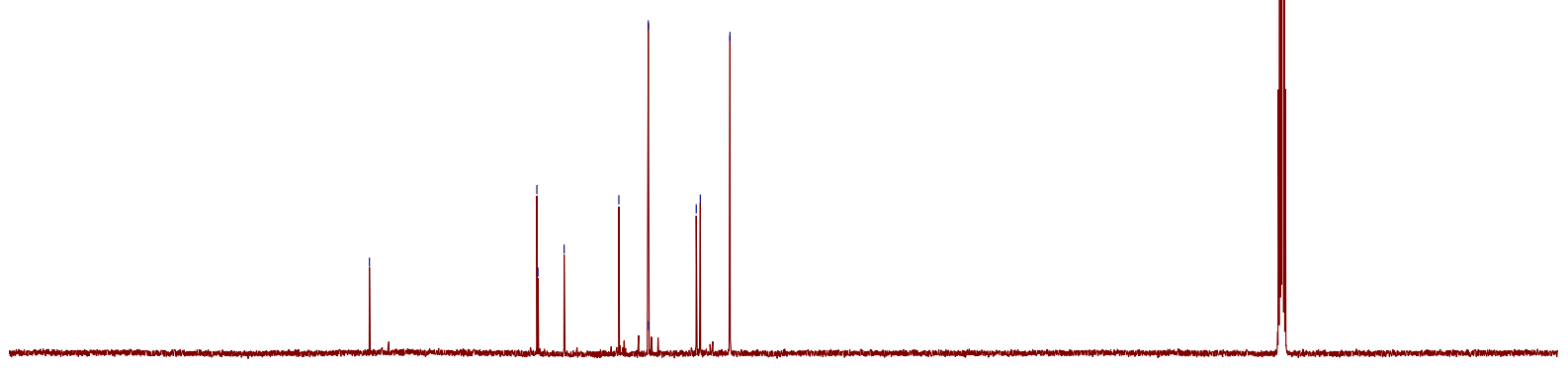

$200 \quad 190 \quad 180 \quad 170 \quad 160 \quad 150 \quad 140$

130

$20 \underset{f 1(\mathrm{ppm})}{110} 100$

90

80

60 
20210510-1048-B500_B.14-50.10.fid

Ref TS1-139 C

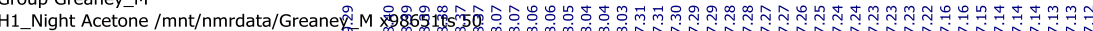
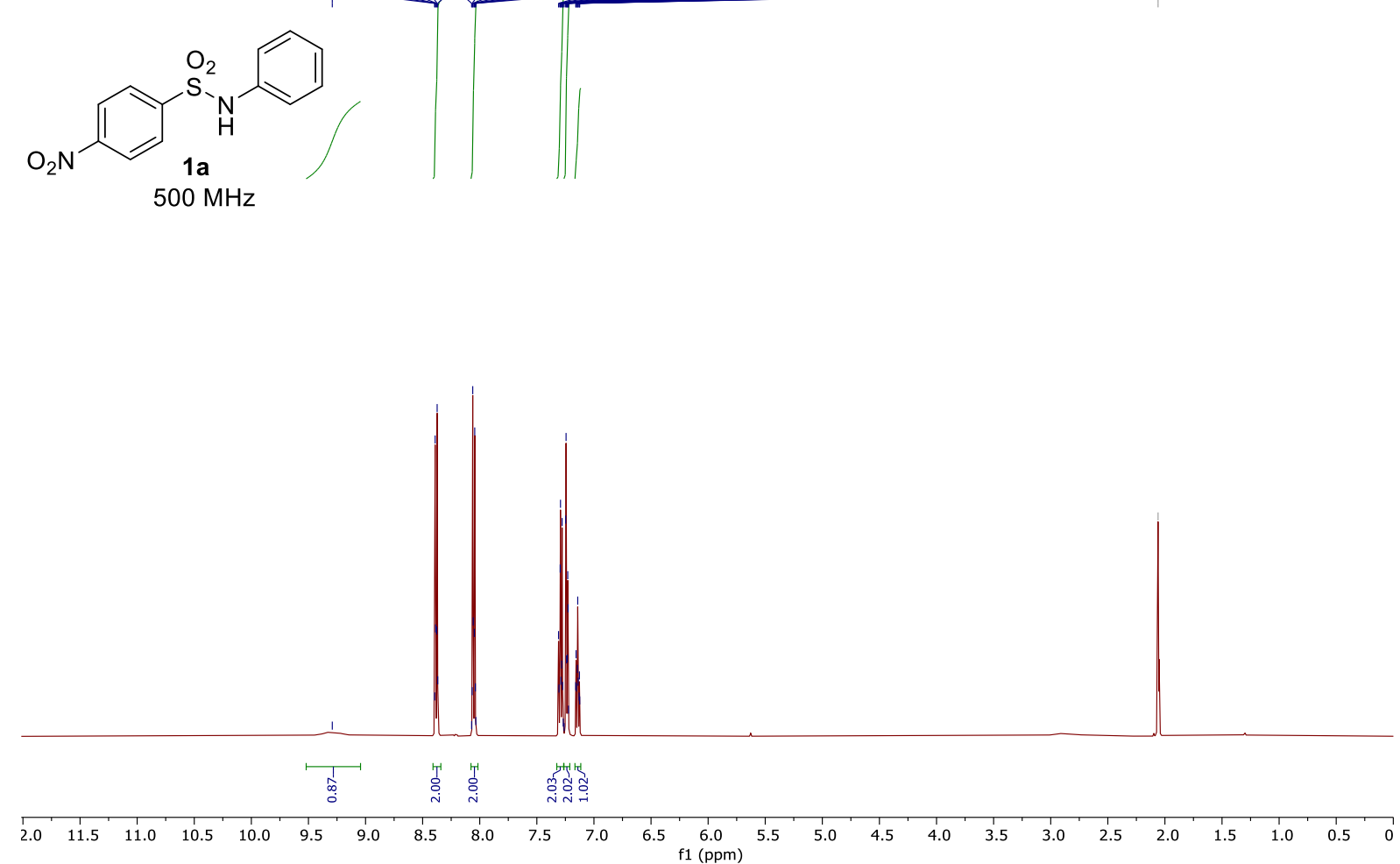

20210510-1048-B500_B.14-50.11.fid

Ref TS1-13p C

Group Greaney_M

Greaney+M

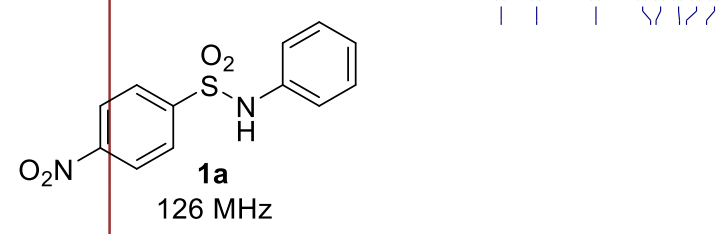

$126 \mathrm{MHz}$

$\begin{array}{llllllllllllllllllllll}210 & 200 & 190 & 180 & 170 & 160 & 150 & 140 & 130 & 120 & 110 & 100 & 90 & 80 & 70 & 60 & 50 & 40 & 30 & 20 & 10 & 1\end{array}$


20210524-1146-B500_B.14-30.10.fid

Ref TS2-67

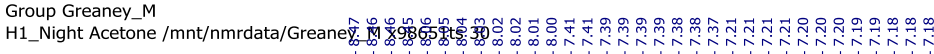<smiles>CN(c1ccccc1)S(=O)c1ccc([N+](=O)[O-])cc1</smiles><smiles>C=CCCCC</smiles>

$500 \mathrm{MHz}$

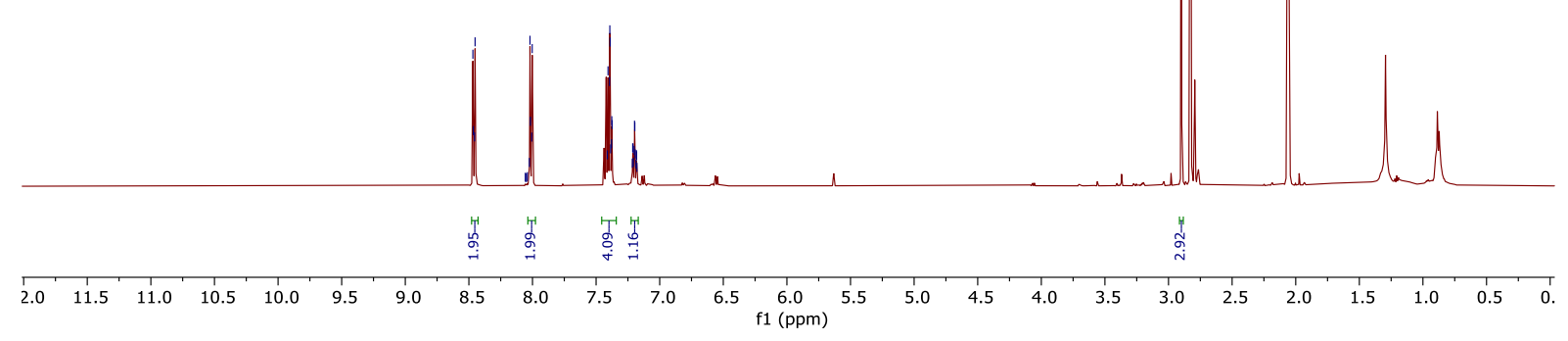

20210524-1146-B500_B.14-30.11.fid

Ref TS2-67

Group Greapey_M

C13_CPD_Night256 Acene/mnt/nmat<smiles></smiles> 
20211116-1421-B500_B.14-22.11.fid

Ref TS3-64FC

Group Greaney_M

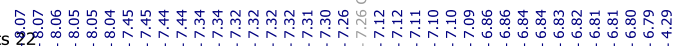<smiles>Nc1ccccc1Sc1ccc([N+](=O)[O-])cc1</smiles>

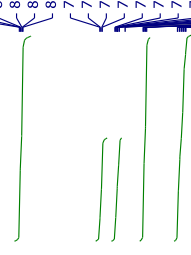

$500 \mathrm{MHz}$

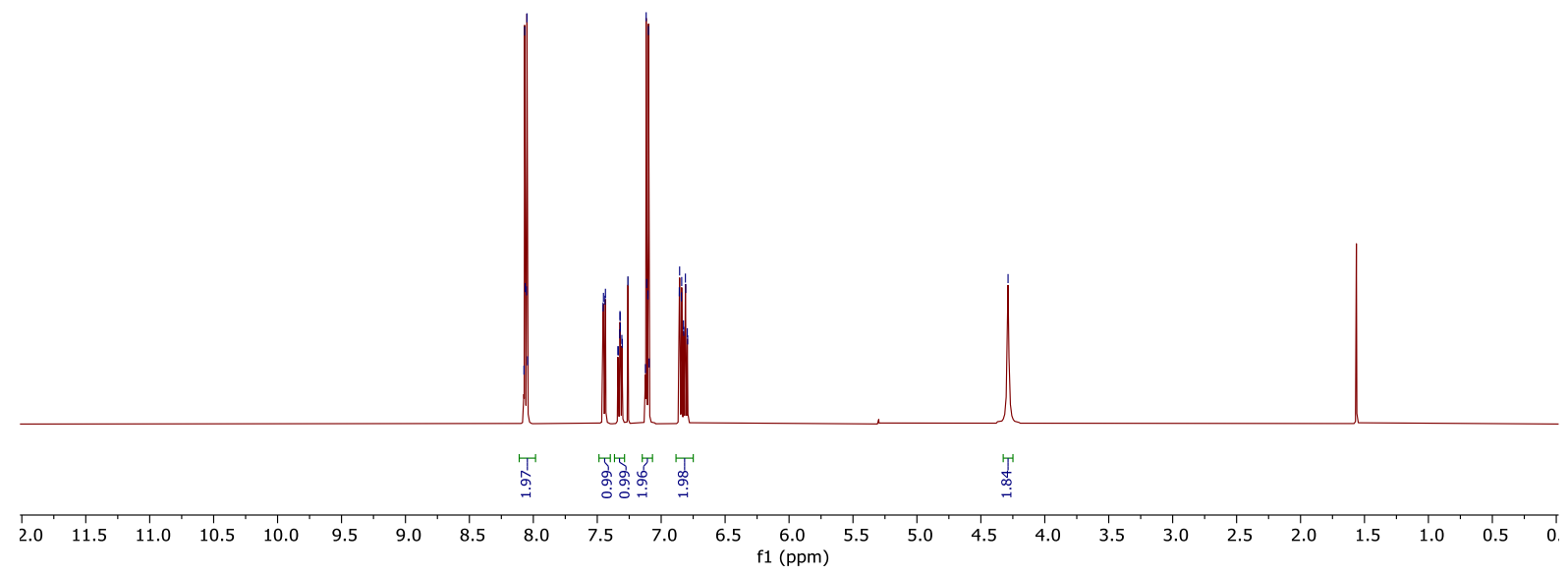

20211116-1421-B500_B.14-22.12.fid

Ref TS3-64FC

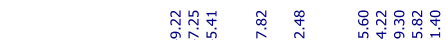

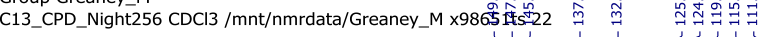<smiles>Nc1ccccc1Sc1ccc([N+](=O)[O-])cc1</smiles>

$126 \mathrm{MHz}$
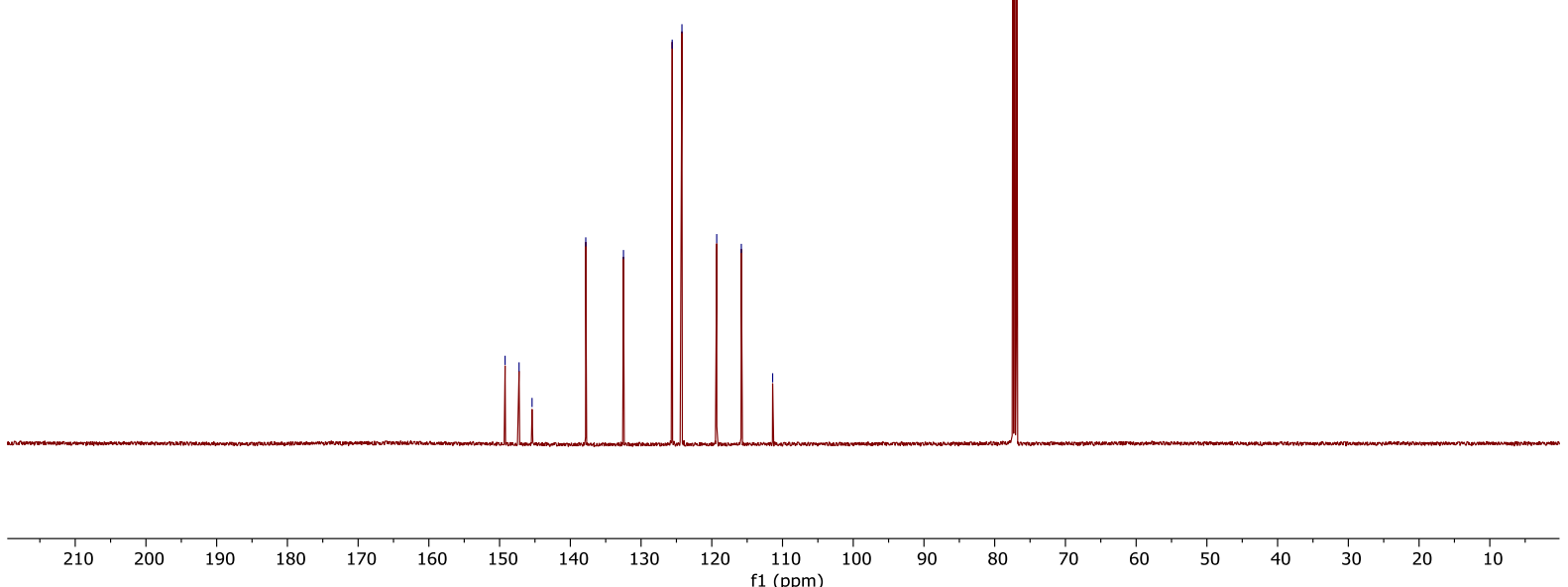
20211116-1422-B500_B.14-23.11.fid

Ref TS3-66FC

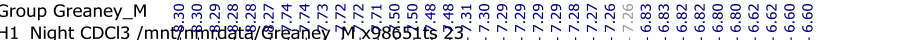

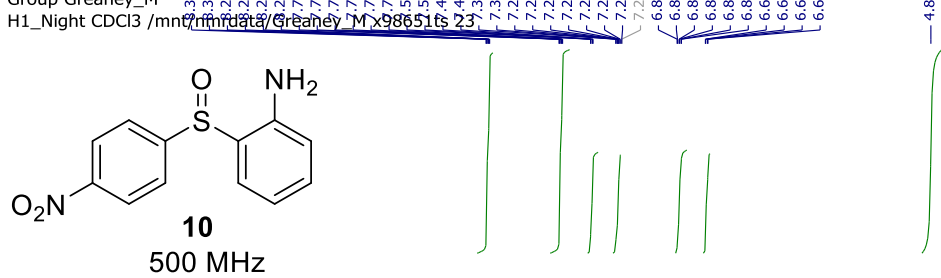

$500 \mathrm{MHz}$

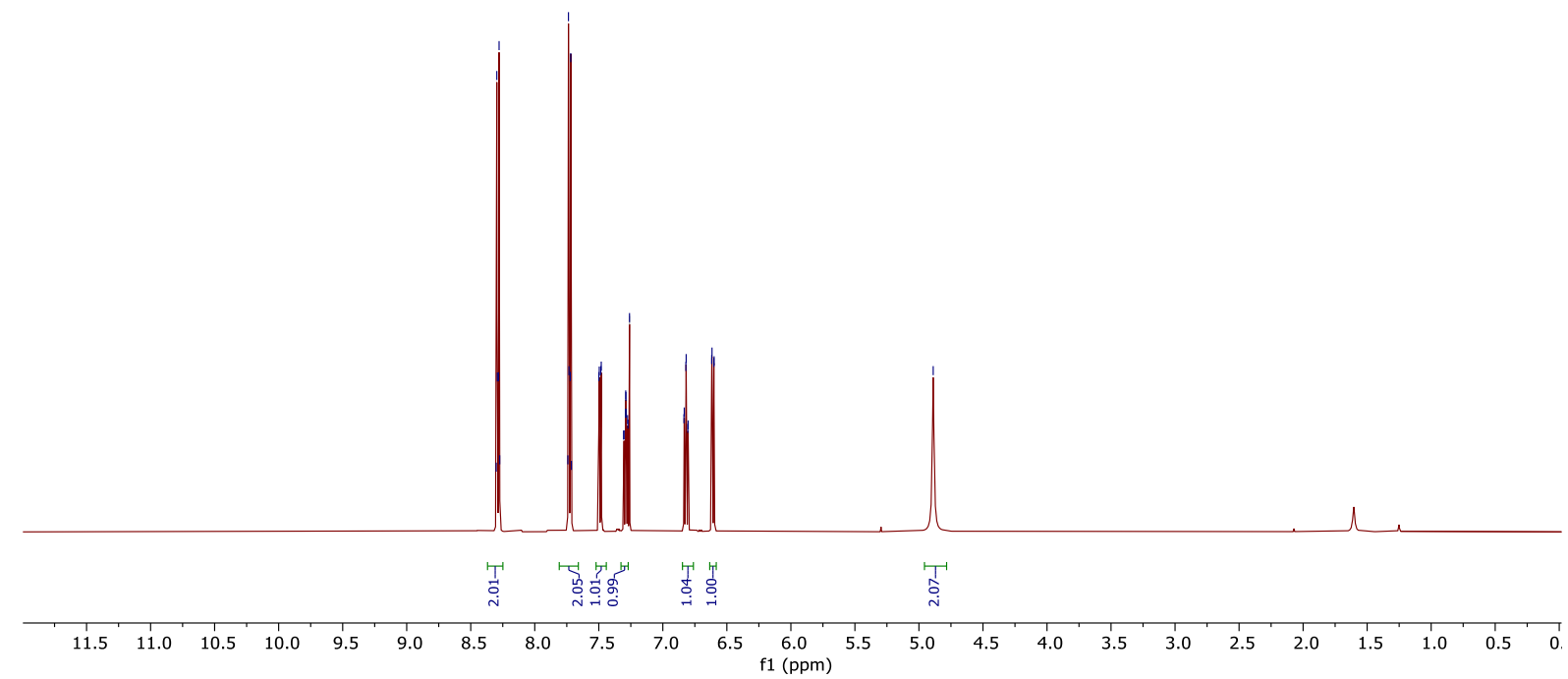

20211116-1422-B500_B.14-23.12.fid

Ref TS3-66FC

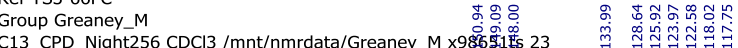<smiles>Nc1ccccc1S(=O)c1ccc([N+](=O)[O-])cc1</smiles>

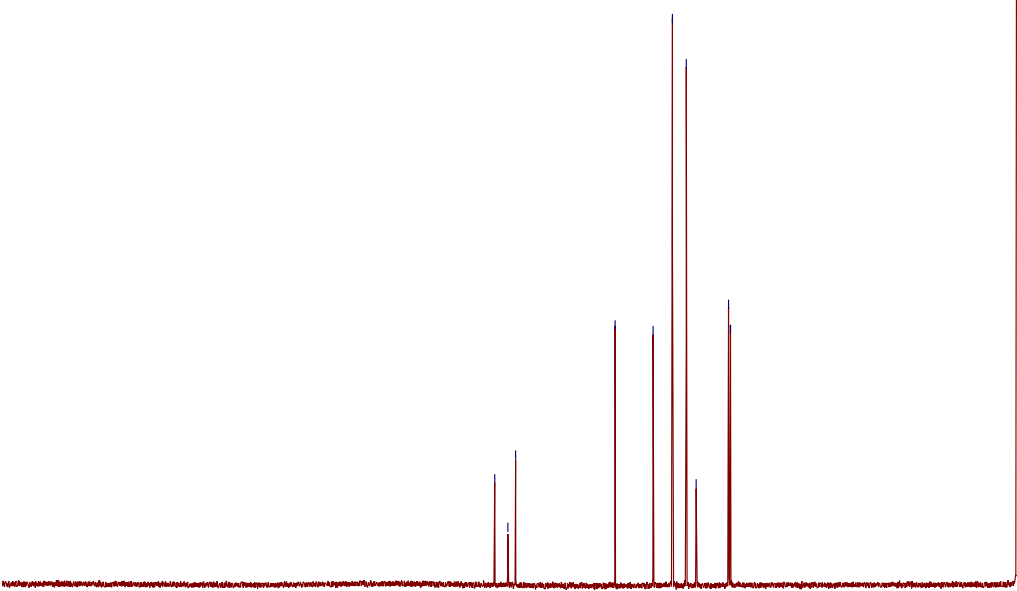

$20 \quad 210 \quad 200 \quad 190$

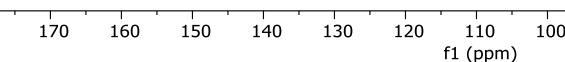


20210106-1609-B400_B.12-15.10.fid

Ref TS1-72 2D

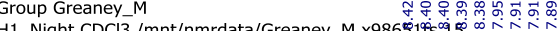

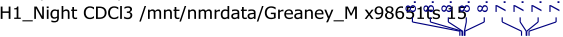<smiles>COS(=O)c1ccc([N+](=O)[O-])cc1</smiles>

$400 \mathrm{MHz}$

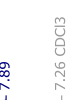

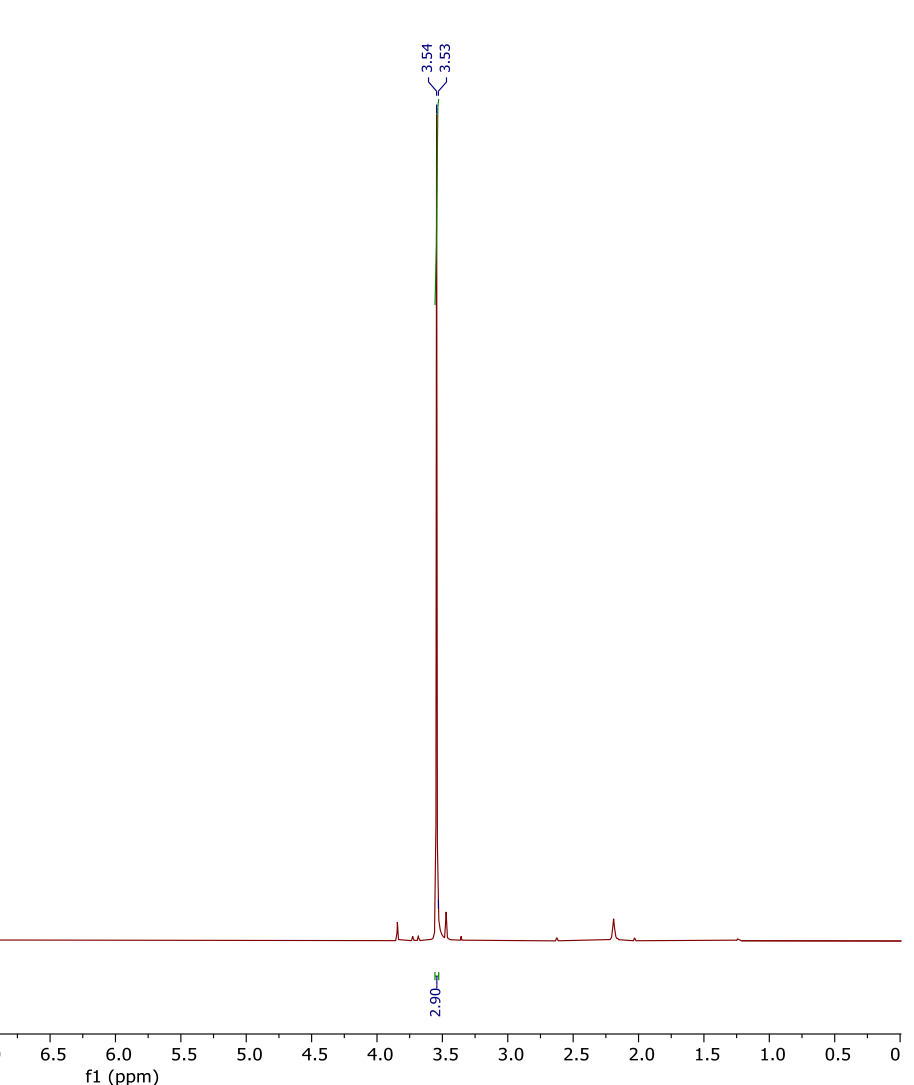

20210105-1645-B400 B. 12-13.12 fid

Ref TS1-72 Carbon

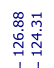

min.

C13 CPD Night256 CDCl3/mnt/nmrdata/Greaney M x98651ts 13<smiles>COS(=O)c1ccc([N+](=O)[O-])cc1</smiles>

$101 \mathrm{MHz}$

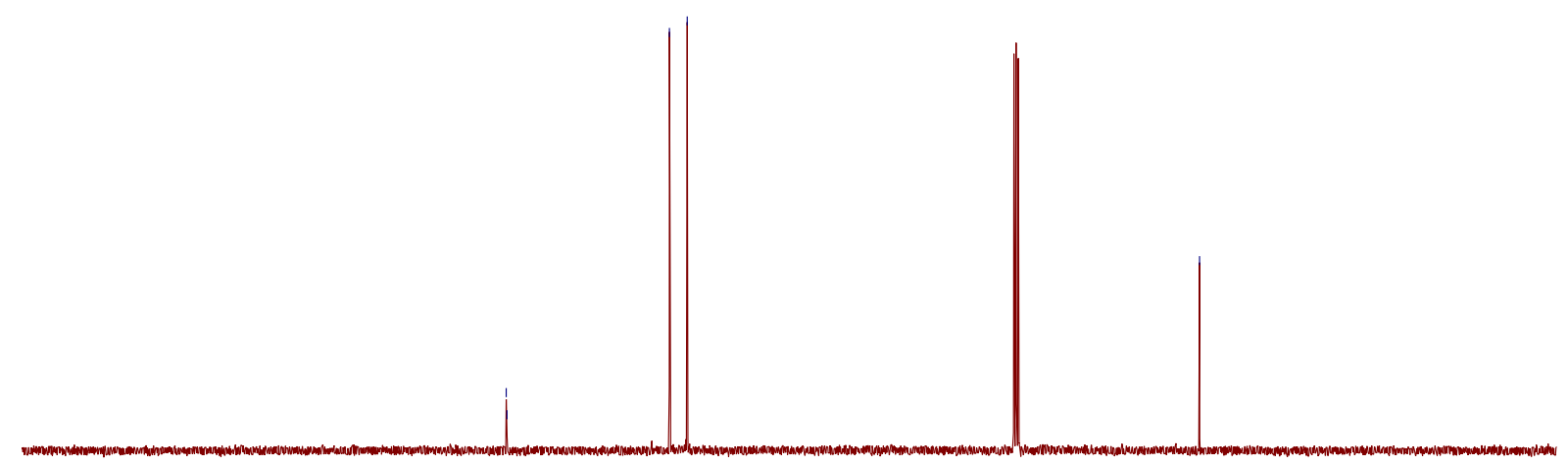

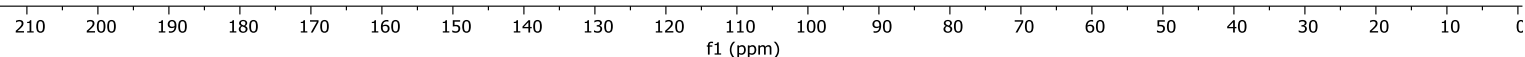


20210910-1215-B500_B.14-7.10.fid

Ref TS2-174FC

H1_Night DMSO/mnt/nmrdata/Greaneyginp<smiles>CC(=O)OCc1ccc([N+](=O)[O-])cn1</smiles>

$400 \mathrm{MHz}$
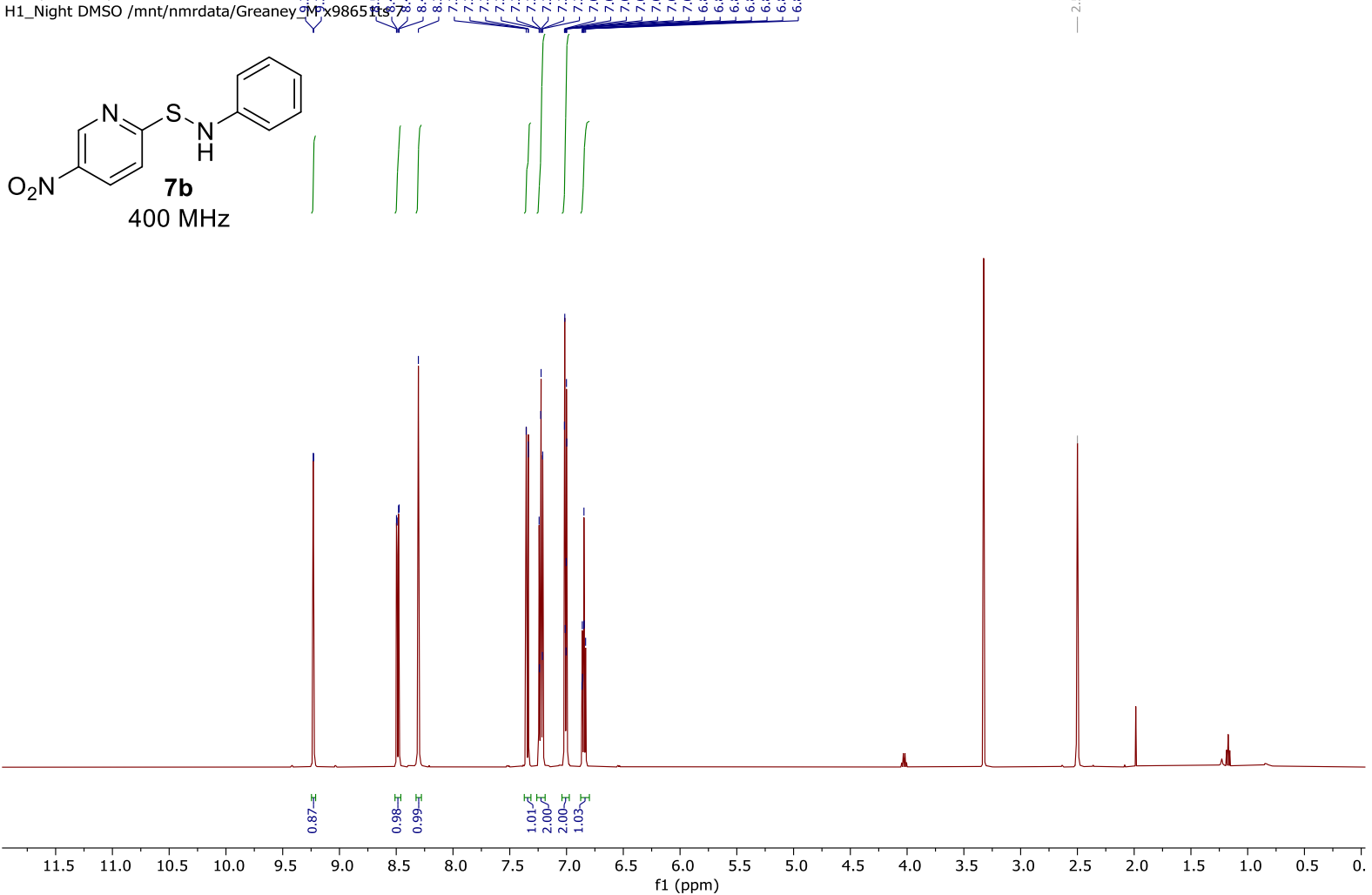

20210910-1215-B500_B.14-7.11.fid

Ref TS2-174FC

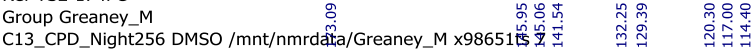<smiles>[R][14c]1cc([N+](=O)[O-])cnc1SNc1ccccc1</smiles>

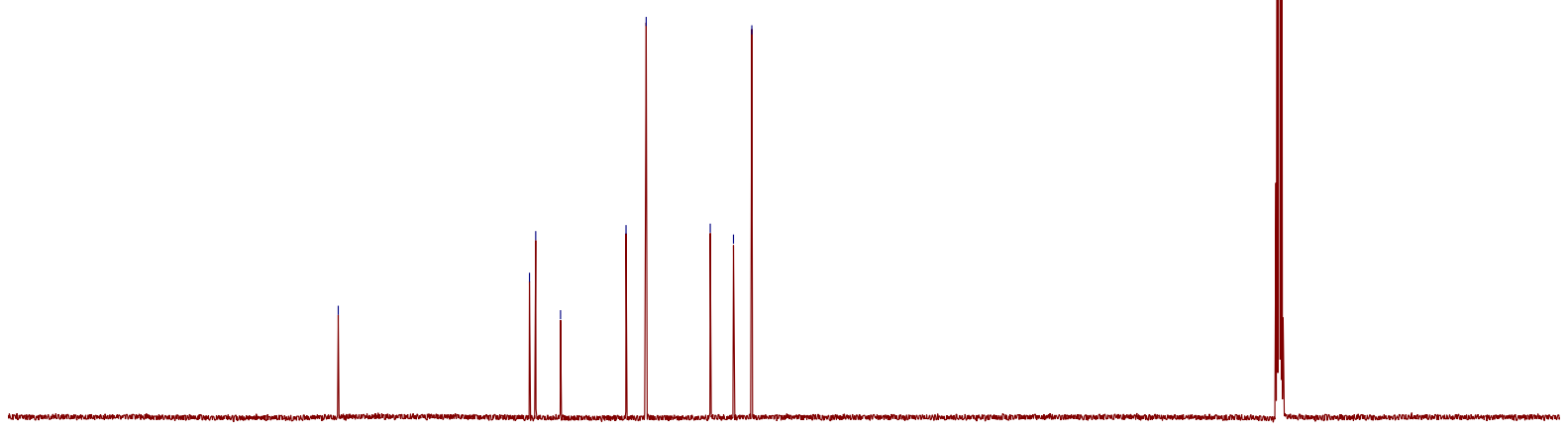

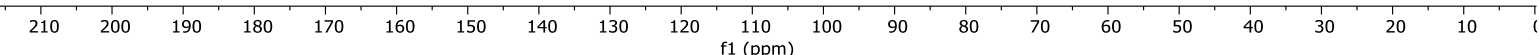




\section{References}

1 C. Hervieu, M. S. Kirillova, T. Suárez, M. Müller, E. Merino and C. Nevado, Nat. Chem., 2021, 13, 327-334.

2 WO2008157802 (A1), 2008.

3 X. Ding, M. Huang, Z. Yi, D. Du, X. Zhu and Y. Wan, J. Org. Chem., 2017, 82, 5416-5423.

4 M. O. Akram, A. Das, I. Chakrabarty and N. T. Patil, Org. Lett., 2019, 21, 8101-8105.

5 EP2450348 (A1), 2012.

6 Y. Fang and R. C. Larock, Tetrahedron, 2012, 68, 2819-2826.

7 N. Barot, T. Shaikh and H. Kaur, New J. Chem., 2017, 41, 5347-5354.

8 J.-Q. Di, M. Zhang, Y.-X. Chen, J.-X. Wang, S.-S. Geng, J.-Q. Tang and Z.-H. Zhang, Green Chem., 2021, 23, 1041-1049.

9 M. Uchino, K. Suzuki and M. Sekiya, Chem. Pharm. Bull. (Tokyo), 1979, 27, 1199-1206.

10 M. Datta and A. J. Buglass, Synth. Commun., 2012, 42, 1760-1769.

11 M. Harmata, P. Zheng, C. Huang, M. G. Gomes, W. Ying, K.-O. Ranyanil, G. Balan and N. L. Calkins, J. Org. Chem., 2007, 72, 683-685.

12 S. W. Youn, T. Y. Ko and Y. H. Jang, Angew. Chem. Int. Ed., 2017, 56, 6636-6640.

13 Y. Shimotori, M. Hoshi, M. Murata, N. Ogawa, T. Miyakoshi and T. Kanamoto, Heterocycl.

Commun., 2018, 24, 219-230.

14 P. Brownbridge and I. C. Jowett, Synthesis, 1988, 1988, 252-254.

15 J. L. García Ruano, J. Alemán, C. Fajardo and A. Parra, Org. Lett., 2005, 7, 5493-5496.

16 F. A. Davis, A. J. Friedman, E. W. Kluger, E. B. Skibo, E. R. Fretz, A. P. Milicia, W. C. LeMasters, M.

D. Bentley, J. A. Lacadie and I. B. Douglass, J. Org. Chem., 1977, 42, 967-972. 